\title{
NEGOTIATING “CULTURAL” IDENTITIES: \\ EXPLORATION OF YOUNG ASIAN WOMEN'S RACIALIZED-GENDERED \\ EXPERIENCES AND MENTAL WELL-BEING
}

\author{
by \\ Maria Krisel Abulencia \\ BScN, Ryerson University, 2013 \\ A thesis \\ presented to Ryerson University \\ in partial fulfillment of the \\ requirements for the degree of \\ Master of Nursing \\ in the program of Nursing
}

Toronto, Ontario, Canada, 2019

(C) Maria Krisel Abulencia, 2019 


\section{Author's Declaration}

I hereby declare that I am the sole author of this thesis. This is a true copy of the thesis, including any required final revisions, as accepted by my examiners.

I authorize Ryerson University to lend this thesis to other institutions or individuals for the purpose of scholarly research.

I further authorize Ryerson University to reproduce this thesis by photocopying or by other means, in total or in part, at the request of other institutions or individuals for the purpose of scholarly research.

I understand that my thesis may be made electronically available to the public. 


\begin{abstract}
NEGOTIATING “CULTURAL” IDENTITIES:

EXPLORATION OF YOUNG ASIAN WOMEN'S RACIALIZED-GENDERED EXPERIENCES AND MENTAL WELL-BEING
\end{abstract}

Maria Krisel Abulencia

Master of Nursing, Ryerson University, 2019

Explanations of mental health outcomes of Asian women in diaspora are often invoked through the concepts of "culture" and "acculturation" with little consideration of asymmetric power relations and structural influences. Informed by critical theories and a narrative approach, this secondary research analyzed data of an exploratory study with fourteen 1.5 and second generation young Asian women living in Toronto, Canada. Research results include: (1) identity construction is a complex process shaped by participants" experiences in both the "mainstream" and "heritage" contexts; (2) participants' encounters of racialized-sexism, microaggressions, and "Othering" contributed to varying degrees of internalized oppressions, which compromised their mental well-being; (3) family support and community engagement enhanced participants' positive self-concept and resilience; and (4) current conceptualizations of "acculturation" and "enculturation" are inadequate as they negate the structural determinants of integration. Nursing research, policy and practice must consider the effects of structural factors in identity construction and mental well-being. 


\section{Acknowledgements}

I am incredibly grateful to the fourteen participants who shared their stories and inspired this work. My sincere gratitude also goes out to the original CIV team (Jessica, Chandni, Jennifer, Lily, and Vanessa) for such a memorable and joyous research experience.

I had the extraordinary good fortune of having a supervisory committee whose affirming guidance made this journey much brighter. I am indebted to my thesis supervisor and mentor, Dr. Josephine P. Wong, for her relentless faith in me and support beyond university walls. Josephine, thank you for showing me what it means to choose courage over comfort and how to live mindfully with humility, humour, and generosity. I consider it a great honour to be your student and bear witness to your unwavering commitment to social justice and equity. My deepest gratitude also goes out to my committee members: Dr. Mandana Vahabi and Dr. Coralee McLaren. Mandana, thank you for your crucial wisdom that gave me the resolve to challenge my own thinking for the better. Your fierce enthusiasm gave me the energy to believe in myself and my work. Cora, I am grateful for your kindness and insightful guidance. Thank you for encouraging me to become more forthright in my voice and to chase my curiosities. Special thanks to my external examiner, Dr. Janet Yamada, for her review and feedback.

I would like to acknowledge the graduate fellowship provided by Ryerson University for making this research possible.

Much love to my brilliant friends and fellow grad students whose patience, cheers of encouragement, and humour sustained me. My heartfelt appreciation to my life-long teachers, Mr. G and Tita Vicky, for their steadfast support throughout all these years.

I am grateful to Nicole and Kate, for the joy of sisterhood and friendship. Being your Ate has been a source of great meaning. To Julius, for your love and unfiltered goodness. You are my source of strength and clarity. Thank you for being there in every page - this thesis and I are better for it.

Lastly, I owe everything to my parents, Lourdes and Arnel, who like many immigrants, sacrificed everything so my sisters and I could walk a better path. Thank you for supporting this journey in great and small ways. This work is a tribute to your love. 


\section{Dedications}

This thesis is dedicated in loving memory of two extraordinary women:

my grandmother,

Zenaida Correa Sta. Cruz

(1931 - 2019)

and my aunt,

Minda Gubatan

(1956 - 2015) 


\section{Preface}

This manuscript-style thesis was prepared under the guidance of my supervisory committee: Dr. Josephine P. Wong, Dr. Mandana Vahabi, and Dr. Coralee McLaren at Daphne Cockwell School of Nursing, Ryerson University.

This thesis is a study of secondary analysis that drew on the focus groups and individual interviews collected for the study, Cultures, Identities, and Voices (CIV) - An Exploratory Study on the Growing Up Experiences, Relationships and Sexual Health of Asian Young Women in Toronto, led by Dr. Josephine Wong in 2012-2013. As the undergraduate project coordinator. I actively engaged in participant recruitment and data collection.

This secondary analysis focused on interrogating the concepts of culture, acculturation, and enculturation by examining the processes of identity construction among the participants and how these processes affected their mental well-being. I undertook data analysis and writing under the critical guidance of the thesis supervisory committee, who met with me regularly and provided me with feedback, which I incorporated into the finalized draft of the thesis.

Chapters 4 and 5 of this thesis are presented in the form of two distinct manuscript-style chapters. Each chapter will be revised into manuscripts based on journal requirements for submission with the support and guidance of the thesis committee members. 


\section{Table of Contents}

Author's Declaration .....................................................................................................................

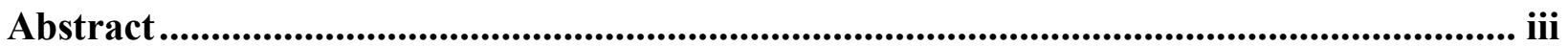

Acknowledgements ..........................................................................................................................iv

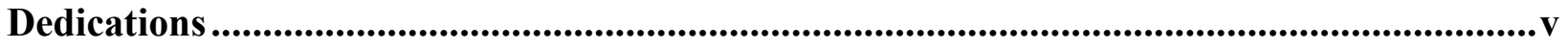

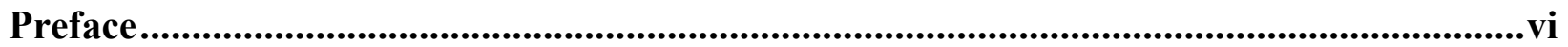

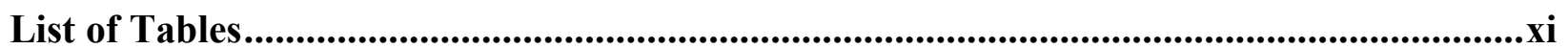

List of Appendices.................................................................................................................................ii

Chapter 1: Introduction ..........................................................................................................1

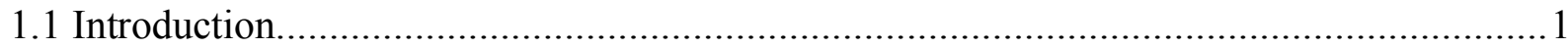

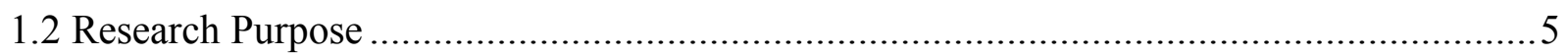

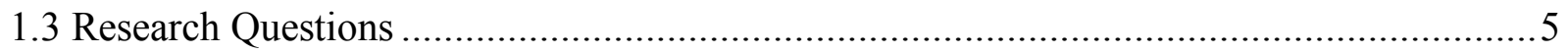

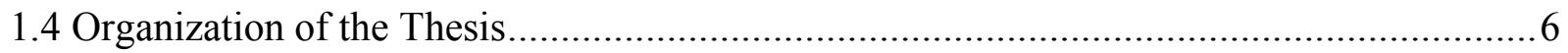

1.5 Summary

Chapter 2: Literature Review ...........................................................................................................8

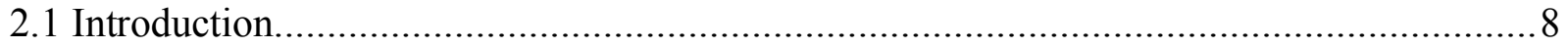

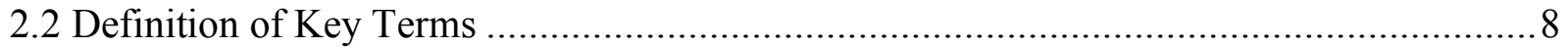

2.3 Construction of "Canadian" Culture and Identity.............................................................. 10

2.4 Dominant Discourse on “Asian” Culture and Identity....................................................13

2.5 Mental Health of Asian Individuals in North America .................................................15

2.6 An Ambiguous Intersection: Racialized-gendered experiences .......................................17

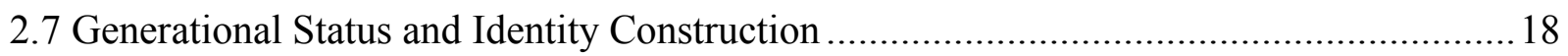

2.8 Acculturation/Enculturation and Young Asian Women's Mental Well-being...................21

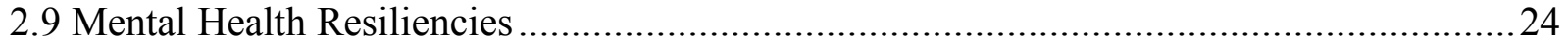

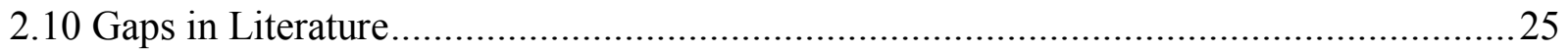




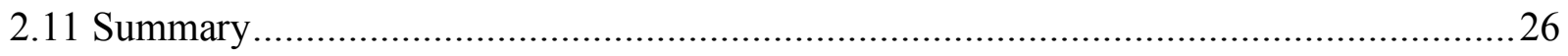

Chapter 3: Methodology ...................................................................................................................28

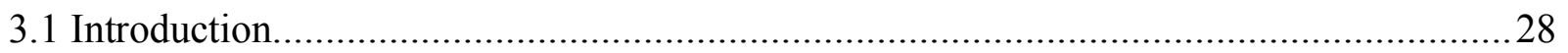

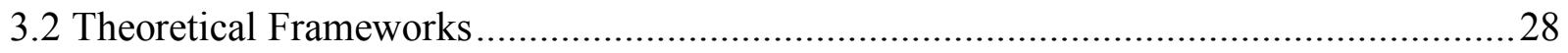

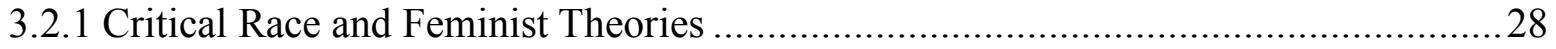

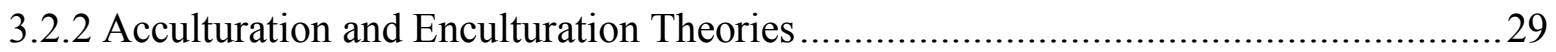

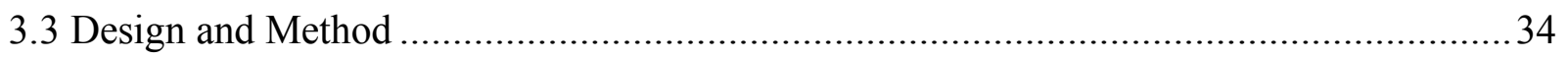

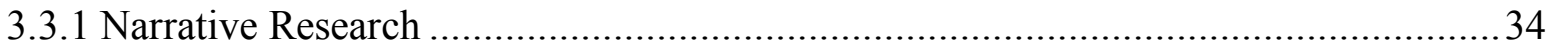

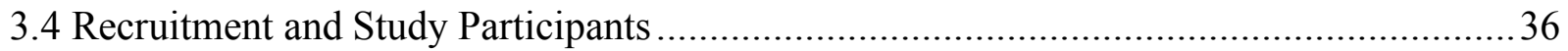

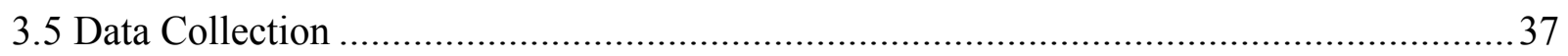

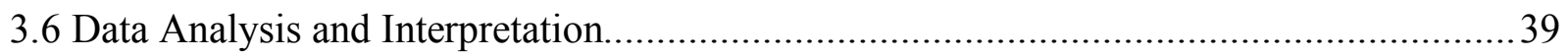

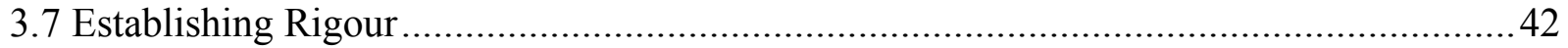

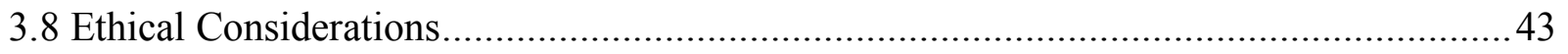

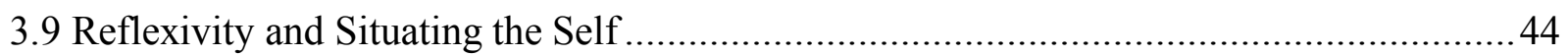

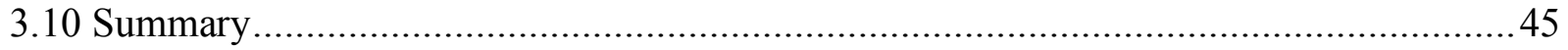

\section{Chapter 4: Racialized-gendered Experiences and Mental Health Vulnerabilities of Young}

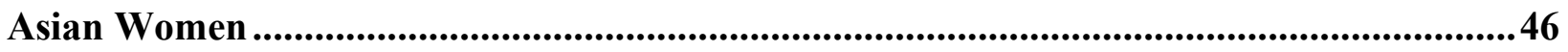

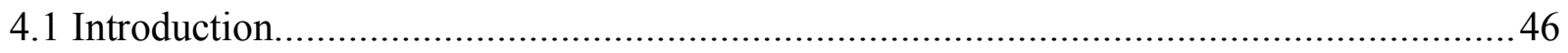

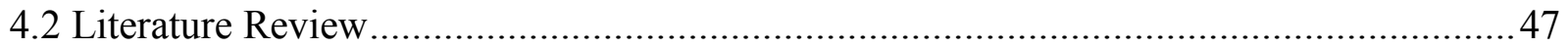

4.2.1 Racism and Asian Mental Health Vulnerabilities ...................................................4

4.2.2 Sexism and Asian Women's Mental Health Vulnerabilities ......................................49

4.2.3 Generational Status and Mental Health Vulnerabilities of Asian women....................50

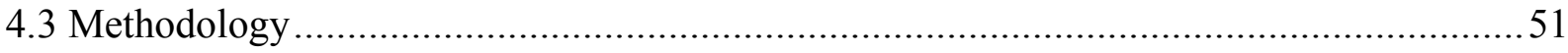

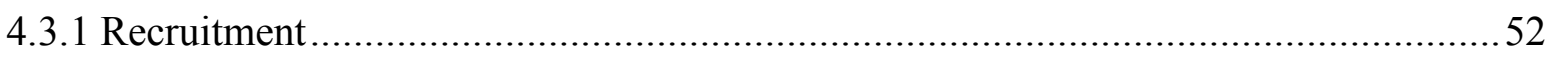

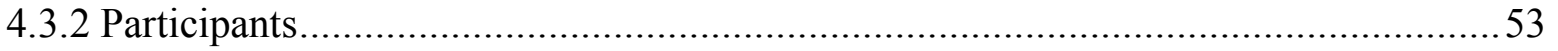

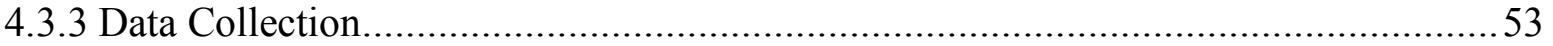

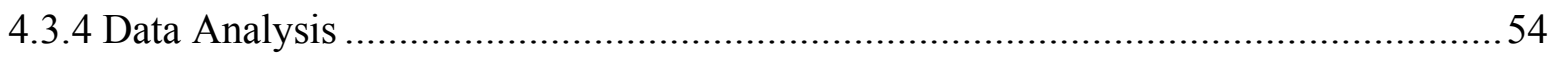




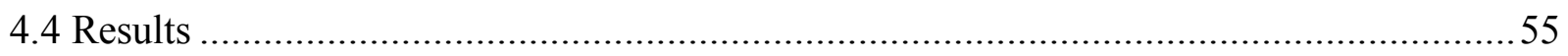

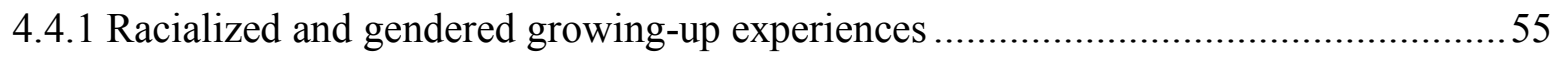

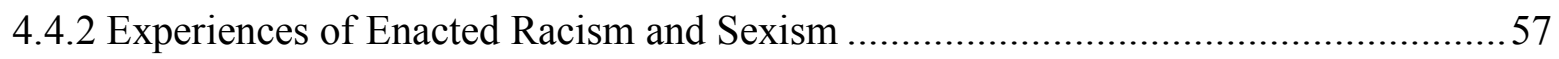

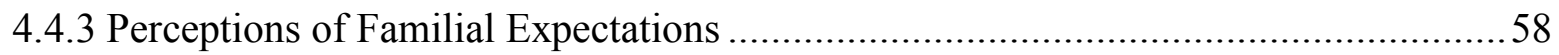

4.4.4 Coping, Resistance and Mental Health....................................................................... 61

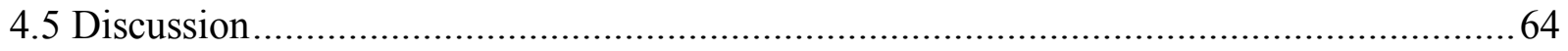

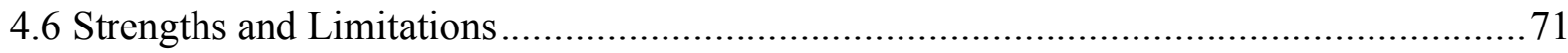

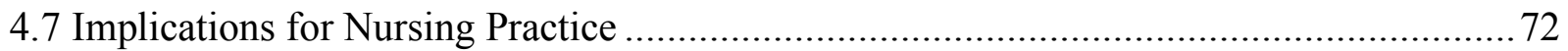

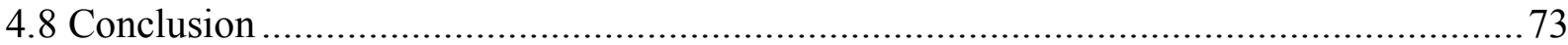

Chapter 5: "Not Canadian Enough": Young Asian Women's Identity Construction and Mental Well-being....................................................................................................................................75

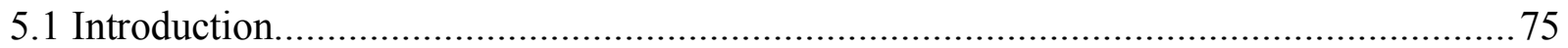

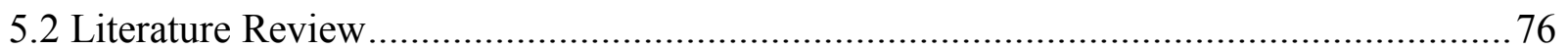

5.2.1 Dominant discourses on "Canadian" and "Asian" cultures .......................................... 76

5.2.2 Current Conceptualization of Acculturation and Enculturation ................................79

5.2.3 Identity Construction and Mental Health of Young 1.5 and 2.0 Asian Women..........8 82

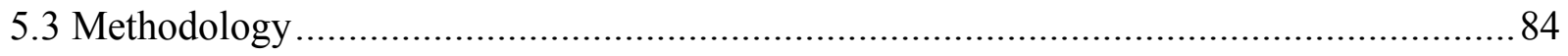

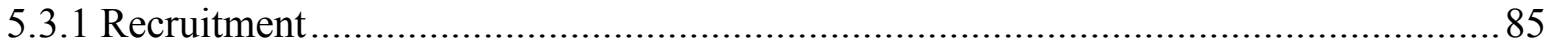

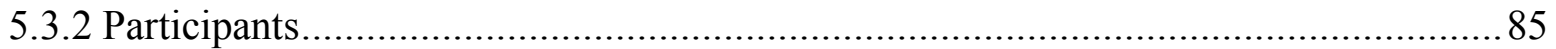

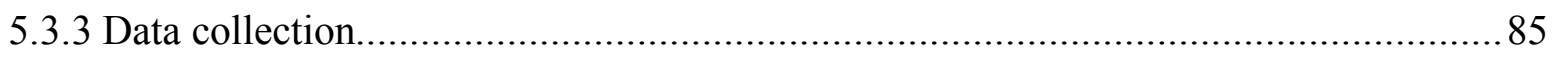

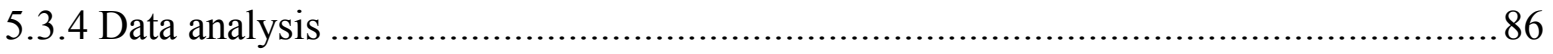

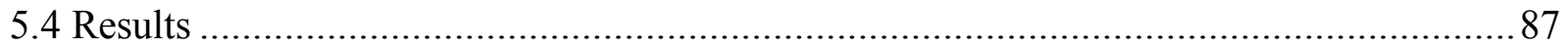

5.4.1 Being "Asian" in the "Canadian" Context: Whiteness as benchmarking.....................87

5.4.2 Being "Fresh-off-the-boat" or "Whitewashed" Asians ............................................... 89

5.4.3 "Not that Asian": Negotiating "acculturation" and Identities.......................................91

5.4.4 Creating In-between Cultures and Identities..........................................................93

5.4.5 Constructing Multiple Situational "Selves" and Mental Well-being ..........................96

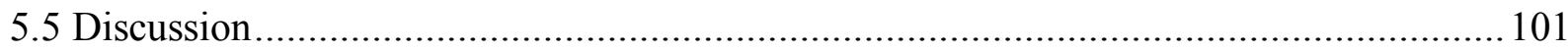




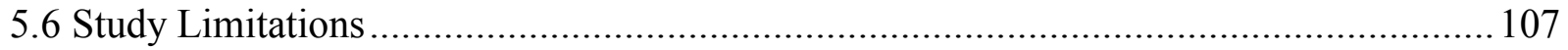

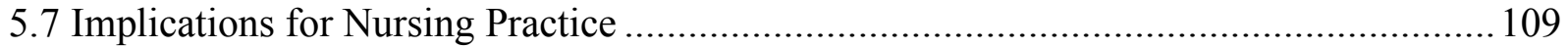

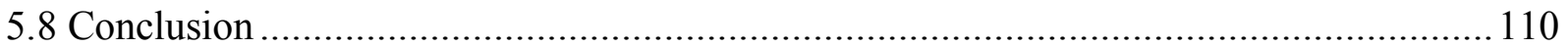

Chapter 6: Conclusion ............................................................................................111

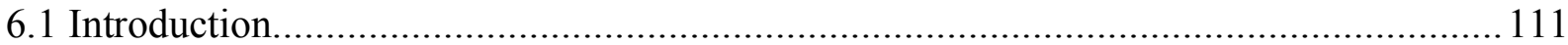

6.2 Overview of Key Insights............................................................................. 111

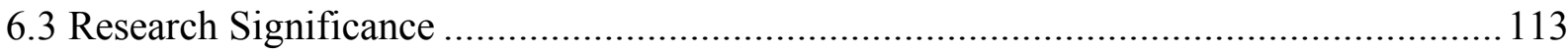

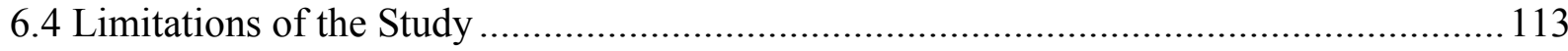

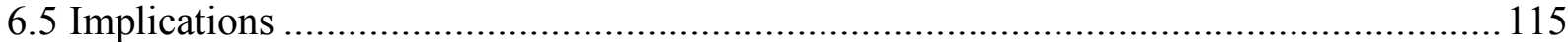

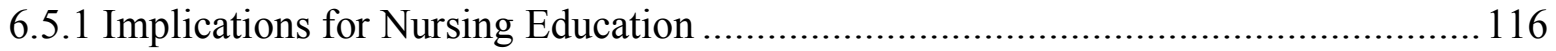

6.5.2 Implications for Nursing Practice.............................................................. 118

6.5.3 Implications for Future Research ........................................................ 119

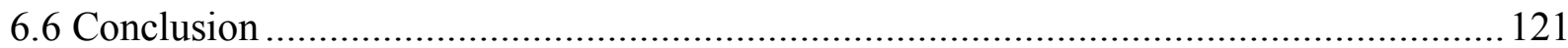

Appendix A: Ryerson University Research Ethics Board Approval Letter ........................ 123

References .................................................................................................................... 124 


\section{List of Tables}

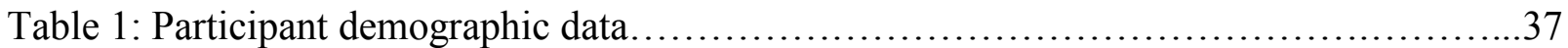




\section{List of Appendices}

Appendix A: Ryerson University Research Ethics Board Approval Letter ...................123 


\section{Chapter 1: Introduction}

\subsection{Introduction}

Canada is recognized as one of the most culturally diverse countries in the world, with over 235,000 new landed immigrants per year (Statistics Canada, 2016). In 2016, Canada's largest group of immigrants originated from Asia, with almost half (48\%) born in South, East, and South-East regions (Statistics Canada, 2017). Of these, 52\% are women and girls who settled in large, urban cities located in Ontario, British Columbia, and Quebec (Chui, 2015). Immigrant women represent over $21 \%$ of Canada's female population - the highest proportion in the last 100 years (Hudon, 2015). Based on the current global immigration trends, the population of immigrant women will continue to grow in Canada.

Of all racialized ${ }^{1}$ immigrant women who choose to migrate and settle in Canada, women from Asia are least likely to self-report good mental health compared to other ethnic groups (Bushnik, 2016). Western ${ }^{2}$ discourse constructs this finding to "traditional"" Asian ${ }^{4}$ cultural dynamics; specifically, the significance of the family and community connectedness, emphasizing family harmony (Leu, Walton, \& Takeuchi, 2011), modesty, and humility (Bernstein, Park, Shin, Cho \& Park, 2011). There is a strong emphasis on not bringing shame or

\footnotetext{
${ }^{1}$ Racialization refers to the "process by which markers such as skin colour and physical features are used to construct or define 'races' as real, different, and unequal in socially, politically and economically significant ways" (Khan, Kobayashi, Lee, \& Vang, 2015, p. 8).

2 "Western" is a broad term that describes, but is not limited to, the social norms, policies, values, beliefs, and political structures that are derived from or associated with Europe. In Western countries, White peoples are positioned in the highest level of hierarchy, while marginalized and racialized groups remain on the periphery (McGibbon \& Etowa, 2009).

${ }^{3}$ The use of the term "traditional" commonly refers to an inherited or established set of practices, meanings, beliefs that have been passed along to others or accepted as historical. This word is problematic as it implies neutrality and assumes legitimacy towards any concept that is deemed "traditional", ignoring the historical, political, social, economic and power hierarchies that have contributed to the values or worthiness attached to the notion of "tradition".

${ }^{4}$ For the purpose of this research, the term "Asian" refers to individuals, customs, practices, or beliefs that originate from, or is associated with South, East and/or Southeast Asian regions. Asians are diverse group of people with shared and/or different customs, languages, beliefs, and practices.
} 
embarrassment to the family, and admittance of mental health problems to non-family members is often discouraged or dismissed (Augsberger, Yeung, Dougher, \& Hahm, 2015). Additionally, some authors suggest that there is a lack of mental health awareness and/or acknowledgement in some Asian cultures (Augsberger et al., 2015; Lee et al., 2009). Without critical analysis and deconstruction, there is a tendency to invoke "cultural" explanations for the mental health outcomes and behaviours of Asian individuals. This discursive practice perpetuates racist stereotypes while masking the pervasive and broader effects of racism and marginalization on internalized and associated stigma of mental illness that are (re)produced by power relations.

To further explore the cultural and mental well-being of Asian individuals in North America, researchers often refer to the concept of acculturation - broadly defined as the changes that occur when groups of individuals from different cultural backgrounds engage in prolonged contact with one another (Sam \& Berry, 2006). Many researchers consider the acculturation process as a critical variable in the mental health outcomes and behaviours of racialized groups (Miller, Yang, Kayi, Choi, \& Lim, 2011; Hwang \& Ting, 2008). For example, some authors suggest that higher levels of acculturation to the dominant ${ }^{5}$ culture is associated with better mental health outcomes (such as lower stress levels and greater help-seeking behaviour; Miller et al., 2011). On the other hand, other authors suggest that higher levels of enculturation ${ }^{6}$ to individuals' heritage culture is associated with positive psychological functioning (Alamilla, Kim, Walker \& Sisson, 2017; Tummala-Narra, Deshpande, \& Kaur, 2016). These findings offer conflicting discourses on the impact of acculturation and enculturation on Asian individuals'

\footnotetext{
${ }^{5}$ Throughout this thesis, the terms "mainstream" and "dominant" will be used interchangeably to describe individuals, values, and/or practices from White, Western backgrounds that frequently serve as the "locus of normalcy" that ethnic groups deviate from (Kumas-Tan, Beagan, Loppie, MacLeod \& Frank, 2007, p. 555). When individuals or concepts are considered dominant, the notion of privilege follows - which often remains elusive. ${ }^{6}$ Enculturation refers to the (re)learning about and/or maintenance of one's heritage culture (Kim, Ahn, \& Lam, 2009). See Chapter 2 for a more in-depth definition and analysis of enculturation.
} 
mental health. It is important to note that these results negate the existence of unequal power relations across different contexts and social spaces, including the 'dominant' and 'subordinated' cultural spaces that shape one's ability to navigate and (re)construct their identities and mental well-being.

The majority of research on immigrant Asian women's mental health has been focused solely on first ${ }^{7}$ generation Canadians. Less is known about the growing up experiences of their $1.5^{8}$ and second $(2.0)^{9}$ generation children. Limited research on 1.5 and 2.0 generation individuals suggest that they are more likely to "assimilate" into the mainstream or dominant Western culture than their first generation parents (Abouguendia \& Noels, 2001). However, their acculturation experiences and contexts are different, presenting health concerns unique to this population (Suarez-Orozco \& Qin, 2006). For example, common stressors associated with migration and settlement, such as language barriers and employment, may not impact 1.5 and 2.0 generation individuals to the same degree as their first generation counterparts. This may be, in part, due to their socialization and introduction into mainstream education at an early age (Abouguendia \& Noels, 2001). Thus, acculturation experiences during adolescence and early adulthood have greater implications on 1.5 and 2.0 generation individuals' identity development and mental health outcomes (Rajiva, 2006; Goodenow \& Espin, 1993).

The mental health challenges and resilience of 1.5 and 2.0 generation young Asian women may be associated with their experiences in constructing their racialized and gendered identities, whereby they have to reconcile the norms and expectations of both their heritage cultures and

\footnotetext{
${ }^{7}$ First generation refers to individuals born outside of Canada (Statistics Canada, 2018a).

${ }^{8}$ The term 1.5 generation has been used to denote individuals who have migrated and settled in another country when they were children or young adolescents. In this study, 1.5 generation consists of young women who arrived in Canada as children or young adolescent and lived in Canada for more than 7 years at the time of the interview.

${ }^{9}$ Second (2.0) generation individuals are those born in Canada with at least one parent born outside of Canada (Statistics Canada, 2018a).
} 
those of the Canadian mainstream culture (Lee et al., 2009). Crossing cultural boundaries may challenge identity development and lead to emotional, psychological, and mental health issues (Khan \& Cottrell, 2017). Most results, however, do not differentiate or explore how asymmetric power relations, Whiteness ${ }^{10}$, and hegemonic racialized-gendered constructs shape their growing up experiences and mental health vulnerabilities.

There is evidence suggesting that 2.0 and 3.0 generation racialized individuals face greater mental health challenges than their first generation counterparts (Suarez-Orozco \& Qin, 2006). Rajiva (2006) suggests that 1.5 and 2.0 generation racialized individuals live in social contexts wherein they learn of, or are defined as being different ("Othering" ${ }^{11}$ ) within the dominant national fabric. Abouguendia and Noels (2001) argue that because 2.0 generation racialized individuals have greater contact with mainstream contexts at a young age, they are more likely to experience racism and discrimination. Other authors suggest that these individuals are exposed to unique diasporic cultures as their identities are governed by simultaneous and often contrasting pressures from external (mainstream or heritage contexts) or internal influences. As they navigate and reconcile these competing influences, they may experience identity and/or family conflict (Stroink \& Lalonde, 2009), which may cause them to feel marginalized from both their mainstream and heritage cultures (Kim, Brenner, Liang, \& Asay, 2003). Given that 2.0 generation individuals represent almost $18 \%$ of the Canadian population and continue to grow steadily (Statistics Canada, 2017), the paucity of literature on 1.5 and 2.0

\footnotetext{
${ }^{10}$ Whiteness refers to socially and politically constructed dimensions of racism that confers life-long advantages and authorities based on having White skin and in the same vein, exploits and oppresses those who do not (McGibbon \& Etowa, 2009; Kivel, 1996). Further, the everyday normalization of Whiteness becomes the standard from which people of colour deviate from; perpetuating the racialized "Other".

11 The "Other" refers to an individual or group socially excluded from the dominant society. Being "Othered" is the process of imposed differentiation of racialized or marginalized individuals/groups that occurs through discrimination, prejudice, and stereotyping (Rajiva, 2006; Hall, 1997). "Othering" serves the interest of the dominant mainstream society by sustaining Whiteness, privilege, and asymmetrical power relations (Calliste \& Dei, 2000).
} 
generation young Asian women's mental well-being is a considerable concern. There is a critical need to further explicate the growing up experiences of 1.5 and 2.0 generation young Asian women by examining how these experiences shape their identity construction and mental wellbeing.

\subsection{Research Purpose}

The overall goal of this study is to address the current gaps in the literature on young Asian women's mental well-being. Specifically, I aimed to explore the racialized and gendered growing up experiences of 1.5 and 2.0 generation young Asian women in Canada by interrogating the dominant discourses of culture, acculturation, enculturation, and how it shaped their mental well-being. This secondary analysis drew on existing data that was collected for the Cultures, Identities, and Voices (CIV) study with young Asian-Canadian women ${ }^{12}$.

\subsection{Research Questions}

Based on my interests in interrogating the theoretical concepts of culture, acculturation, enculturation, and mental health, my primary research question was:

1. How do the racialized and gendered growing up experiences of 1.5 and 2.0 generation young Asian women affect their mental well-being?

To answer this question, I explored the following sub-questions:

1. What are the growing up experiences of 1.5 and 2.0 generation young Asian women in Toronto, Canada?

\footnotetext{
${ }^{12}$ As mentioned in the preface, I was the research coordinator for the CIV study. The CIV study focused on how acculturation/enculturation influenced the identity construction and sexual health of young Asian women. Primary analysis of the results also revealed the impact of acculturation/enculturation processes in the mental well-being of the participants and as such, inspired the questions of this current study.
} 
2. How do the dominant discourses of "Canadian identity" and "culture" shape the intertwined social identities and growing up experiences of young 1.5 and 2.0 generation young Asian women?

3. How do young 1.5 and 2.0 generation young Asian women's growing up (acculturation/enculturation) experiences affect their mental health?

I used a narrative approach to conduct secondary data analysis to gain a deeper understanding of how young Asian women negotiate their identities and mental well-being at the intersection of heteronormative gender and cultural expectations. I drew on an integrated analytical framework of critical narrative analysis (CNA), Critical Race Theory (CRT) and Critical Feminist Theory (CFT) to interrogate how these dominant discourses are implicated in the racialization process to: empower or marginalize young Asian women, shape their perceived identities and social practices, and affect their mental well-being. Further, I critically ${ }^{13}$ applied existing theoretical concepts of acculturation and enculturation to elucidate how the growing up experiences of young Asian women are influenced by their social positioning based on race, gender, class, and migration histories.

\subsection{Organization of the Thesis}

In Chapter 2, I present a review of existing literature, which will serve as a backdrop to contextualize the study purpose and questions. It also includes an overview of key definitions relevant to the study.

Chapter 3 describes the methodological and theoretical orientation of this study. It includes a detailed description of theoretical frameworks, recruitment strategies, data collection, data analysis, and ethical considerations.

\footnotetext{
${ }^{13}$ The term 'critical' refers to the philosophical approach that seeks to closely interrogate social, historical, and discursive structures that shape power relations (Ford \& Airhihenbuwa, 2010; McGibbon \& Etowa, 2009)
} 
Chapters 4 and 5 present the results of the study. Chapter 4 focuses on the participants' racialized-gendered growing up experiences and their impact on mental well-being. Chapter 5 draws on these racialized-gendered experiences to interrogate broader discourses of "culture", “acculturation”, and "enculturation”, which are prominent concepts used by researchers to explore the mental well-being of Asian communities. Each of these chapters are organized in a manuscript-style format with its own introduction, literature review, methods, results, discussion, and conclusion sections. Although each manuscript consists of a distinct theme and analysis, there are some overlaps between the chapters' methodology sections. These chapters will be revised and submitted for publication upon completion of this thesis.

I conclude the thesis with Chapter 6 by presenting the study limitations and implications for nursing education, practice, and research.

\subsection{Summary}

In this chapter, I introduced the background, significance, and purpose of this research study. The following chapter offers a review and critique of themes that arose from existing literature on culture, acculturation, enculturation, and mental health of young Asian women. 


\section{Chapter 2: Literature Review}

\subsection{Introduction}

In this chapter, I report on a literature review conducted to situate the current study. The chapter begins with definitions of key terms, followed by an exploration of the relevant themes from existing literature: 1) current discourses on Canadian and Asian cultures and identities, 2) mental health of Asian individuals in North America, 3) the nature of racialized-sexism, 4) generational status and identity construction, 5) young Asian women's acculturation/enculturation experiences and mental well-being, and 6) their mental health resiliencies. Although this chapter does not offer an exhaustive review, it highlights the critical gap in Canadian research on acculturation, enculturation, and mental well-being of 1.5 and 2.0 generation young Asian women.

\subsection{Definition of Key Terms}

This section outlines definitions of key terms that are important to the thesis. These research terms and discourses are associated with competing definitions and interpretations in the field of nursing, psychology, and social science. Hence, it is critical to define and clarify the intended application of these terms throughout the thesis.

Asian: The term "Asian" refers to individuals, customs, practices, or beliefs that originate from, or is associated with South, East and/or Southeast Asian regions. Asians are diverse group of people with shared and/or different customs, languages, beliefs, and practices. Each group and its individuals have unique histories and experiences in their migration, socioeconomic, political, and educational backgrounds.

Acculturation: Broadly defined, acculturation is a complex process wherein individuals from ethnically and culturally diverse backgrounds interact with a cultural community different 
from their own heritage (Chirkov, 2009a; Ngo, 2008). Much of what scholars understand about the acculturation process is influenced by John Berry's theoretical framework (Ngo, 2008). Berry proposes four acculturation strategies that immigrants use within intergroup encounters: integration (heritage cultural maintenance and acquisition of host ${ }^{14}$ culture is sought), assimilation (acquisition of host culture is preferred and heritage culture is rejected/avoided), separation (heritage culture is preferred and host culture is rejected/avoided), and marginalization (rejection of both heritage and host culture; Sam \& Berry, 2006). A more in-depth, critical analysis on acculturation is provided in Chapter 3 .

Canadian: On paper, the term Canadian is supposed to represent all people with citizenship status in Canada. In reality, the term Canadian refers to the dominant group representing majority of Canada's population. In a White settler society like Canada, the term "Canadian" refers primarily to individuals of Caucasian European (i.e. English and French) background. In Canada, power and privilege are afforded to these "Canadians" more frequently than racialized minorities despite citizenship status. It is important to note that class and socio-economic status dictate access to certain privileges and outcomes even for Euro-Caucasian individuals. However, Eurocentric dominance embedded in Canadian structures and institutions continue to perpetuate injustice on the basis of "race". It confers Euro-Caucasians access to social goods and services at the expense of racialized minorities (McGibbon \& Etowa, 2009).

Culture: Culture is a construct comprised of salient social systems (visible artifacts, behaviours, beliefs, values) through which individuals and communities organize and interpret

\footnotetext{
${ }^{14}$ The term "host" is commonly used in acculturation psychology to describe the dominant culture or country that is "receiving" the acculturating individual (Sam \& Berry, 2006). While I believe that this term further perpetuates Othering by inferring that racialized migrants are merely "foreigners" in "hosted" dominant Canadian spaces, the use of this terminology is prevalent in acculturation scholarship. The term "host" conceptualizes "Canadian" culture as the norm and portrays racialized immigrants as "guests" or "foreigners". Henceforth, this oppressive term will be replaced with the word "mainstream" when referring to the "Canadian" culture, which is frequently associated with White individuals from Euro-Western backgrounds.
} 
their social worlds (Rudmin, 2009; McGibbon \& Etowa, 2009). Multi-dimensional in nature, "culture" can shift, interact, and change when people interact with one another (McGibbon \& Etowa, 2009).

Enculturation: The process of socialization and maintenance of one's heritage cultural norms, values, and practices (Yoon et al., 2013; Kim et al., 2009).

Mental well-being: A state of well-being wherein individuals recognize their own potential, cope with life stresses, work productively and contribute to their community (World Health Organization, 2014). It may also shape the way we interpret the world, make choices, connect with others, and handle emotions. Mental health is influenced by many factors including biology, family history, socio-economic status, life experiences, gender, sexual orientation, ethnicity, geographic, and political locations among others (U.S. Department of Health \& Human Services, 2017).

\subsection{Construction of "Canadian" Culture and Identity}

The construct of "culture" is predominantly defined as a social system of ideas, values and practices through which individuals conceptualize their own worldviews and values (McGibbon \& Etowa, 2009). It also functions as a mode through which we interpret our experiences and maintain our taken-for-granted assumptions. There is extensive literature on the pervasive influence of culture on individuals' health and health practices, which ascribes such behaviours and outcomes to individual-level cultural explanations (Levesque \& Li, 2014).

Historically, European imperialism played an influential role in Canada's nation building as a British colony, relying on immigration as a labour source to build its infrastructures (Bakan \& Stasiulus, 1995). Racist and exclusive immigration policies were developed and maintained to support the building of a White British colony that was, and still is, deeply racialized because it 
privileges British middle- and upper-classes. From the late 1800s to the 1960s, consistent mechanisms of racism were embedded in immigration policies, favouring immigrants who could assimilate into and embrace the Canadian society: those who were educated and spoke English (Fernando \& Rinaldi, 2017). Immigrants, who did not socially reproduce European heritage on the basis of their ethnicity, were subjugated to substandard, low-paying working conditions (Roy, 2008), denied the right to vote, work in professional occupations, or own property (McGibbon \& Etowa, 2009). Despite the changes in Canadian immigration policies in the 1960s, justification for excluding specific groups of migrants (those who are considered undesirable) continue to be legalized within the immigration points system (Tannock, 2011) and Temporary Foreign Worker program (Sharma, 2006) to name a few. This selection process played an influential role in Canada's racialized ethnic, gender, and class landscape that continues to affirm the centrality of Whiteness and cultural inferiority of the racialized Other (Fernando \& Rinaldi, 2017; Kumas-Tan, et al., 2007).

The intensification of globalization also contributed to the growing diversity of Canada's cultural and ethnic makeup. This phenomenon is responsible for minimizing borders and barriers imposed by geographic distance, which leads to the deconstruction and reconstruction of societal and individual identities (Bodislav, Bran \& Iovitu, 2015). Although the advantages of globalization are often contested, some authors assert that it propelled a shift towards appreciating cultural differences, evidenced by the development of the Canadian Multiculturalism Act of 1985 (Minister of Justice, 2014) which promotes ethno-cultural diversity and harmony (Lee \& Hebert, 2006). For example, Canada's international reputation as a multicultural state has been claimed as a source of pride by many Canadians, who believe that cultural diversity is characteristic of the "Canadian" identity (Grant, 2016). This supports 
contemporary discourse that Canada is a pluralistic, safe, friendly, and democratic country, full of freedom and opportunity (Lee \& Hebert, 2006). However, this discourse conceals the social hierarchy and inequities experienced by different racialized ethnic groups in Canada.

On a broader note, values such as individualism and freedom of expression, and traits such as being adventurous, daring, and verbally expressive have been associated with the "Canadian" identity (Stroink \& Lalonde, 2009). The "Canadian" national identity has also been constructed by features of mass culture. Considered today as a landmark of popular culture, Molson's “I Am Canadian" beer campaign in 2000 featured a passionate declaration for Canadian nationalism and its distinctive identity: peacekeeping, diversity, and hockey (Macgregor, 2003). Hockey is a symbol used to protect and demarcate the Canadian identity from other countries and promote a sense of community among its citizens (Watson, 2017). Similarly, outdoor wilderness endeavours such as camping, snowboarding, and family cottages are perceived as iconic features of the Canadian physical and cultural landscape. Such traditions continue to be encouraged and passed on through generations (Stevens, 2013). At the same time, the depiction of the Canadian "great outdoors" is often only associated with faces of White and Western heritage.

Studies that explore the experience of immigrants navigating the Canadian culture also provide insight into what values or traits they perceive as distinctly "Canadian". Romantic involvement is frequently normalized and expected within the Western adolescent experience (Dhariwal \& Connolly, 2013). As such, some immigrant parents equate association with the Western Canadian culture to increased sexual promiscuity (Suarez-Orozco \& Qin, 2006). In a study conducted with Chinese immigrant adolescents, respondents encountered a more "liberal" Canadian teaching style - characterized by friendlier teachers, easier educational materials, less homework, and more emphasis on creativity, independence and sociable personalities (Li, 2009). 
Conversely, it can be argued that this perception may not be an ideal approach for some individuals and represent a weakness in the Canadian educational system.

The growing up contexts in which young Asian women are socialized are entrenched by these dominant "Canadian" cultural identities. These are taken-for-granted discourses that reinforce ongoing colonial domination and invisibility of Whiteness in Canada. By assuming these traits, practices, hobbies, and beliefs as distinctly "Canadian", it marginalizes those who do not identify as such. Without recognizing the history and legacy of colonization, multiculturalism approaches continue to recreate oppression for Indigenous peoples, racialized immigrants (or those considered as Other), and maintain claims of neutrality (Kumas-Tan et al., 2007). As the number of 1.5 and 2.0 generation individuals reporting "Canadian" as one of their ethnic identities continues to increase (Lee \& Edmonston, 2010), there is a need to deconstruct this notion of "cultural identity" and how it privileges certain values and perspectives associated with the dominant "Canadian" ideology.

\subsection{Dominant Discourse on “Asian” Culture and Identity}

To gain a deeper understanding of how young Asian women's racialized-gendered growing up experiences affect their mental health, we must examine the sociocultural contexts in which such processes occur. Although 1.5 and 2.0 generation young Asian women are exposed to diverse ways of living in Canada, their growing up experiences are primarily conditioned by their interactions with their parents and families (Li, 2009). What follows is a Western perspective: scholars often perceive Asian parenting as authoritarian and hierarchical, defined by high parental pressures and expectations for their children to perform culturally acceptable and desirable behaviours (Choi, Kim, Pekelnicky, Kim, \& Kim, 2017). Examples of such behaviours include practicing emotional restraint (Lipsicas \& Makinen, 2010), achieving academic 
excellence (Tummala-Narra et al., 2016; Yoon et al., 2017), maintaining familial and filial obligation (Vu \& Rook, 2012), conforming to social norms to preserve social harmony (Shea \& Yeh, 2008), and retaining of cultural Asian heritage (Rhee, Chang, \& Rhee, 2003).

Some researchers indicate that like most contemporary cultural communities, patriarchal structures persist within the institution of family. It accords fathers the control and decisionmaking authority for the family, while mothers assume the responsibility of raising children and maintaining the home (Vu \& Rook, 2012). Patriarchy influences the division of labour in the family and drives the gendered roles and norms their children are socialized into (Vu \& Rook, 2012). For example, daughters are expected to maintain chastity, obedience, and care for the family and the home (Chang, Wong, Liu, \& Tran, 2017), whereas sons are encouraged to pursue higher education and occupational aspirations (Vu \& Rook, 2012). This "cultural" conceptualization of gender may present mental health and identity development challenges as daughters are primarily expected to transmit cultural and familial values instead of exploring other avenues of expression (Gartner, Kiang \& Supple, 2014).

Despite the dominant Western depiction of Asian parents being restrictive and authoritarian, it is plausible that Asian youth perceive parental "control" as an indirect expression of warmth, love, and care (Choi et al., 2017). However, Choi et al. (2017) suggest that when children acculturate to the Canadian culture, they are more likely to view their parents' guidance and parenting practices as excessive or emotionally distant, resulting in intergenerational conflict and negative mental health outcomes. This conflict may be compounded by parents' expectation for their children to retain their heritage cultural values, while concurrently mastering the skills required to succeed in a country that promotes liberal individualism (Lee et al., 2009; Rhee et al., 2003). 
While only one of many, this Western perspective on Asian cultures dominates the scholarly literature. These stereotypical portrayals of Asian families are based on dominant White knowledge that functions to maintain oppression and power-differences among racialized groups. In particular, this discourse on "Asian” culture promotes benchmarking that uses Whiteness as the "Canadian" norm or standards to demarcate racialized minority groups as deviant and inferior. These assumptions perpetuate racist stereotypes and conceal the broader historical, socio-political, economic, and cultural contexts that shape these discourses on Asian culture.

\subsection{Mental Health of Asian Individuals in North America}

Issues such as racism and ethnic discrimination are known to have pervasive impacts on mental health and wellness, but continue to remain largely invisible (Lee \& Ahn, 2011). The "model minority" stereotype portrays Asian Americans/Canadians as a uniformly successful and well-adjusted ethnic group in all their academic, work, and socio-political endeavours (Tummala-Narra et al., 2016). Western contemporary discourse depict Asian Americans/Canadians as wealthy, academic high-achievers who, unlike other racialized minorities, triumphed over racial challenges (Ho, 2014). Their diligent work ethic and achievements have been lauded as the norm which all minorities must strive for despite their class, migration, gendered, and generational contexts (Ho, 2014). As a divisive tool, this "model minority" myth legitimizes and (re)produces oppression among Asians and other racialized minorities. By way of homogenous categorization, this notion also ignores how racism and discrimination contribute to mental health disparities experienced by Asian communities and thus, negates their mental health needs (Yoon et al., 2017; Tummala-Narra, Alegria, \& Chen, 2012). Further, the label invokes harmful stereotypes and unrealistic expectations, which 
reinforces their prevalence and have harmful implications to the mental health and growing up experiences of young Asian women (Yoon et al., 2017).

Contrary to the "model minority" label, some studies suggest that Asian Americans are more likely to experience mental health issues than other ethnic groups (Gupta, Leong, Valentine, \& Canada, 2013). For example, relative to their White counterparts, Asian American youth are at greater risk for depression (Kalibatseva, Leong, Ham, Lannert, \& Chen, 2017; Young, Fang, \& Zisook, 2010), self-injury, and suicide (Tummala-Narra et al., 2016). Despite these experiences, scholars have recognized the underutilization of mental health services among Asian adults and college students in North America (Shea \& Yeh, 2008; Abe-Kim et al., 2007). It is important to note, however, that the broad and global stigma attached to mental health continue to influence patterns of service utilization. As such, service underutilization is not only limited to this group, but is also common among other ethnic groups (Derr, 2015). This finding has been attributed to various factors. Of these, "cultural values" been the most dominant explanation among researchers exploring Asian immigrant and non-immigrant individuals' attitudes towards mental health service utilization (Chen, Kazanjian, \& Wong, 2009; Abe-Kim et al., 2007; Fung \& Wong, 2007). Others suggest that "traditional" Asian norms and values, which emphasize hierarchy, collectivism, emotional restraint, and obedience, may discourage individuals to seek help for mental health challenges in effort to "save face", avoid being burdensome to others (Alamilla et al., 2017), and minimize the appearance of inadequate family support (Shea \& Yeh, 2008). The perceived difference between dominant "Western" approaches and "traditional Asian" values towards mental health illness and treatment perpetuates a dichotomous perspective. It constructs "Western" mental health discourses as the norm from which the Asian "Other" deviates from. Hence, the practice of reducing and associating help- 
seeking behaviours to cultural determinants ignores the complexity of how other socio-cultural, economic, and demographic factors impact Asian individuals' service utilization for mental health.

Based on my review of this literature, the majority of scholars ascribe Asian individual's underutilization of mental health services to cultural factors. Relatively few studies consider how systemic and institutional barriers, such as income, accessibility, availability, racism, discrimination, and broader mental health stigma impact mental health attitudes and outcomes among Asian populations (Fung \& Wong, 2007). By excluding such factors in exploring the mental health of young Asian women, this practice puts the onus on the individual, reinforces individual-based interventions, and diverts attention away from structural contexts that construct their growing up experiences (Viruell-Fuentes, 2007). Additionally, it is critical to recognize that while there are cultural values and norms commonly observed across Asian cultures, there is great intra-group variability that is often neglected and overlooked (Ruzek, Nguyen, \& Herzog, 2011; Fung \& Wong, 2007).

\subsection{An Ambiguous Intersection: Racialized-gendered experiences}

It is widely acknowledged that women's gender, ethnic, and socioeconomic positioning locate them at various levels of oppression that are upheld and legitimized by dominant institutional structures and power hierarchies (Pyke \& Johnson, 2003). Gender also appears to be associated with mental health issues; in particular, women are more likely to internalize mental health symptoms compared to their male counterparts (Alamilla et al., 2017; Ying \& Han, 2007). Within this gendered climate, dominant stereotypes that portray Asian women as "highly sexualized, eroticized, and objectified" (Tajima, 1989, as cited in Chang et al., 2017, p. 262) may be internalized by the women themselves, reinforcing their influence in social discourse (Yoon et 
al., 2017). Dominant assumptions about gender dynamics in the Western mainstream and Asian heritage worlds paint a dichotomous view and influence young Asian women's constructions of "femininity" that are not only gendered, but also racialized.

Additionally, some authors assert that "traditional" Asian values of familial harmony and subservience have a more pronounced effect on Asian women than men because women bear the pressure of maintaining cultural continuity, especially in the face of a new mainstream culture (Leu et al., 2011). For example, in a study of 1573 Asian American women, Leu et al. (2011) reported that high ethnic and/or cultural identity is positively correlated to depression and anxiety. The multiplicity of findings implies that experiences from both mainstream and heritage groups place Asian women at risk for greater mental distress. From a critical perspective, however, these mental health experiences should be analyzed in the context of racist, patriarchal, and colonial practices that deny women from agency and liberation (Hewitt, 1991).

\subsection{Generational Status and Identity Construction}

Generational status was found to be connected to poor mental health outcomes among Asian populations (Alamilla et al., 2017). Many first generation Asian adults migrate to a new country with an established sense of ethnic identity (Yoon et al., 2017), which may offer some protection from the cumulative negative impact of racial discrimination and stigmatizing experiences on their mental health (Viruell-Fuentes, 2007). The nature of these Othering experiences are comparatively more recent and less frequent in comparison to their 2.0 generation counterparts, thus helping them retain a sense of identity and mental well-being that is not jeopardized by racial discrimination and stigma (Viruell-Fuentes, 2007). Further, other protective factors such as social support networks, "cultural" beliefs and practices, and lower 
rates of substance use have been postulated to explain improved mental health outcomes for first generation individuals. (Salas-Wright, Kagotho, \& Vaughn, 2014).

As such, 1.5 and 2.0 generation individuals are more likely to experience mental health challenges than their first generation counterparts (Islam, Khanlou, \& Tamim, 2014; SalasWright et al., 2014) since they have been exposed to the mainstream culture for a greater portion of their lives, and this exposure shapes their personal and ethnic identity development $(\mathrm{Wu}$, Schimmele, \& Hou, 2012). Under these circumstances, they negotiate their identities as they experience simultaneous and often contrasting influences from their heritage and mainstream cultures (Islam et al., 2014; Wu et al., 2012). While they are less likely to experience their first generation parents' challenges with language barriers or foreign cultural norms (Wu et al., 2012), they are more likely to report pervasive and frequent exposure to racial discrimination (ViruellFuentes, 2007). This may be due to the long-term exposure to interlocking oppression from dominant structures and spaces wherein they are ascribed their status as racialized women from a young age. Racial discrimination negatively shapes pathways (such as behavioral, environmental, psychological, and physiological responses) to mental health outcomes (Williams \& Mohammed, 2013).

Conversely, Liu and Suyemoto (2016) posit that increased length of residence in United States offers an opportunity to better understand racial complexities and dimensions in the dominant culture and perhaps, to develop resources of resilience and resistance against racial discrimination. This suggests that mental health outcomes improve for racialized minorities over time, specifically for 1.5 and 2.0 generation individuals. Nonetheless, these variations in conceptual interpretations call for deeper, more nuanced exploration of the unique ecological 
contexts within which these 1.5 and 2.0 generation individuals grow up and develop their identities and mental health outcomes.

Under circumstances defined by their socio-economic and gendered contexts, 1.5 and 2.0 generation individuals experience dramatic developmental changes during adolescence and young adulthood. In addition, they also navigate their identities within cross-cultural contexts, through a dual process that some scholars refer to as enculturation and acculturation (TummalaNarra et al., 2016; Li, 2009). These concepts will be further explored in the next chapter (see Chapter 3). Racialized youth are faced with navigating multiple cultural spaces wherein they negotiate their achieved and imposed identities, while simultaneously experiencing a wide range of uncertainty and pressures from their parents, communities, and the dominant society (Tummala-Narra et al., 2016; Suarez-Orozco \& Qin, 2006).

Although I argue that the ability and opportunity to participate in mainstream acculturation continue to be dictated by the values and norms of the dominant culture, the growing impact of globalization and social media may facilitate or hinder the degree to which some first generation parents engage in mainstream acculturation. Nonetheless, most first generation parents are more likely to adhere to the values of their heritage culture, a consistent finding across the literature (Choi et al., 2017; Kwak, 2010; Ying \& Han, 2007). To illustrate the complexity, while most 1.5 and 2.0 generation children are expected to maintain similar values and beliefs as their parents, they also experience pressure to fit in with their school peers. Contrasting yet parallel expectations render them vulnerable to family and self-identity conflicts (Tummala-Narra et al., 2016; Ying \& Han, 2007). As such, many Asian youths struggle with connecting with their parents and non-Asian peers as they embody behaviour that is deemed desirable by both ( $\mathrm{Li}$, 2009). Other researchers have found that relative to their non-Asian counterparts, Asian youth 
experience higher levels of social isolation (Rhee et al., 2003) and anxiety, low self-esteem and poor academic performance (Tummala-Narra et al., 2016). Challenges to adolescents' selfdiscovery and identity construction have been ascribed to "traditional" Asian values such as familial cohesiveness and interdependence, which may constrain their capacity for social exploration and socialization (Kwak, 2010; Rhee et al., 2003). However, this perspective ignores the significant role of broader sociocultural contexts such as race, gender, and class-based hierarchies in mediating their cross-cultural growing up experiences and mental well-being.

\subsection{Acculturation/Enculturation and Young Asian Women's Mental Well-being}

The prominence of cultural competency in nursing and allied health literature implies that immigrant health behaviours and outcomes are "culturally" influenced (McGibbon \& Etowa, 2009). To examine this phenomenon, scholars refer to acculturation, which is one of the most widely researched culture-related variables for Asian individuals living in North America (Hwang \& Ting, 2008). Acculturation research on Asian individuals often operationalize this concept to define their level of socialization to the mainstream culture of their settlement country (Kim et al., 2009) and to quantify the extent they have adopted "Western" cultural traits (ViruellFuentes, Miranda, \& Abdulrahim, 2012). Conversely, Kim (2007) argues that the definition of acculturation as the adoption of mainstream culture may not be as relevant to some individuals born in the mainstream country, who are removed from the migration process, not fully immersed in their Asian heritage, and/or are not engaged in cultural maintenance. In response, the concept of enculturation has been adopted and defined as the retention or socialization to one's heritage norms (Kim et al., 2009; Yoon et al., 2013).

Although individuals vary in their responses, the acculturation/enculturation experience is significant to one's identity development (Hilario, Vo, Johnson, \& Saewyc, 2014) and sense of 
self, particularly if a person is a member of an ethnic minority group (Cheng, Carter, \& Lee, 2014). There is also significant evidence that considers the acculturation/enculturation process as a critical variable in the mental health outcomes and behaviours of Asian individuals and communities. To illustrate, some acculturation researchers suggest that Asian individuals with higher levels of acculturation report less psychological distress (Ruzek et al., 2011), less anxiety, higher self-esteem (Farver, Narang, \& Badha, 2002), positive mental health outcomes (Alamilla et al., 2017; Yoon et al., 2017), and are more likely to utilize mental health services (Shea \& Yeh, 2008). Additionally, some authors assert that 1.5 and 2.0 generation youth are developmentally susceptible to faster acculturation than their first generation parents. This is partly due to increased socialization to new sets of mainstream social norms through school, media, and peers (Kwak, 2010; Ying \& Han, 2007; Farver et al., 2002).

Evidence supporting the benefits of acculturation is vastly divergent and inconsistent. Some authors argue that a higher level of acculturation is associated with deleterious outcomes for Asian individuals such as increased stress, depressive symptoms (Choi et al., 2017), family conflict (Choi, Tan, Yasui, \& Hahm, 2016), and perceived discrimination (Tummala-Narra et al., 2016). When 1.5 and 2.0 generation youth adhere to "Western" behaviours, they appear more visible within the mainstream culture and vulnerable to Othering practices that render such behaviours incongruent with their Asian heritage (Choi et al., 2017; Ruzek et al., 2011). They may also experience the culminating effect of the negative portrayal of minorities, thus disrupting positive identity development. This makes them susceptible to mental distress associated with internalized racism and discrimination (Choi et al., 2017; Viruell-Fuentes et al., 2012). 
As young individuals begin to forge an independent identity, highly acculturated youth may face family conflict due to increasing discrepancy with their parents' cultural values and expectations (Kwak, 2010; Ying \& Han, 2007). For example, Vu and Rook (2012) found that highly acculturated Vietnamese American young adults were more likely to report being criticized by their parents and perceive familial conflict than their less acculturated counterparts. Similarly, highly acculturated Korean American youth were more likely to perceive their fathers as controlling and authoritative (Choi et al., 2017). Such findings support existing claims that higher levels of acculturation may be positively correlated with an increase in youth's negative perception of their parents' behaviours (Choi et al., 2017), perpetuating familial discord and deviation. These conflicts may accumulate over time and manifest into negative mental health outcomes for both parents and their children (Ying \& Han, 2007) and persist into late adolescence and young adulthood (Suinn, 2010).

Since acculturation has been the focus of most research efforts, the concept of enculturation has not been extensively researched. Nonetheless, existing enculturation literature presents a contrasting perspective that is inconsistent to our understanding of young Asian individuals' acculturation experiences. Several authors found enculturation to be positively associated with improved mental health outcomes (Alamilla et al., 2017), increased familial and cultural harmony (Choi et al., 2016), self-efficacy and self-esteem (Kim \& Omizo, 2010) among Asian individuals. Specifically, enculturation may foster positive psychological functioning (such as cognitive flexibility), which may buffer against acculturative stress and discrimination for Asian youth through increased self-esteem, social support and effective coping skills (Alamilla et al., 2017; Tummala-Narra et al., 2016). Additionally, highly enculturated Asian youth were more likely to perceive their family relations to be positive, and have a stronger bond 
with their parents (Choi et al., 2017). Enculturation may increase youth's appreciation and identification with their Asian heritage. Feeling a sense of pride and sharing positive feelings towards their co-ethnic peers and parents enhances relationships and positive identity development (Choi et al., 2016; Kim \& Omizo, 2010). It can be asserted that expectations of academic excellence among highly enculturated Asian youth are associated with increased academic motivation and achievement (Fuligni, Witkow, \& Garcia, 2005). These findings imply that young Asian youth's enculturation experiences may not be as detrimental to their identity development and mental health outcomes as portrayed in current literature.

\subsection{Mental Health Resiliencies}

Growing up as visible minority individuals in North America presents unique circumstances that may render young Asian women to the racialized-gendered Othering practices of dominant groups in society. However, it is important to recognize the salience of choice and resilience. Young Asian women are not passive recipients of both heritage and mainstream cultures. Rather, they actively create their own narratives as they navigate between external (i.e. norms defined by heritage/mainstream culture) and internal (i.e. active resistance to discrimination) influences (Li, 2009). Researchers suggest that a growing proportion of visible minority children endorse a stronger ethnic identity than their first generation parents. This suggests that they may deliberately choose to practice their heritage culture despite their growing up in the mainstream community (Choi et al., 2016). They demonstrate resilience by identifying social support and establishing connection with communities and peers from different ethnic backgrounds (Tummala-Narra et al., 2016). When some Asian adolescents experience marginalization, they join co-ethnic peers or create a subgroup to develop a safe space and support one another (Li, 2009). It is important to note, however, that individualist strategies of 
resilience of Asian youth (i.e. personal strengths, individual coping skills) are equally and/or strongly associated with positive mental health outcomes, compared to collectivistic forms of resilience (i.e. social support from family; Lee \& Ahn, 2011). However, in the absence of a critical perspective, these results support the well-documented claims about Asian values such as collectivism and familial piety.

To further illustrate this notion of choice, some researchers suggest that it is possible for Asian individuals to simultaneously maintain and/or adapt their heritage and mainstream cultures (Chae \& Foley, 2010). Other authors posit that simultaneous identification to two cultures may lead to identity conflict and mental distress as Asian youth reconcile two, potentially contrasting, cultural expectations (Stroink \& Lalonde, 2009). In order to negotiate two cultural identities, individuals have the tendency to construe a stark contrast between two cultures and distance themselves from one culture to participate in the other. This ongoing process of cultural reconciliation may lead to reduced identification with either group and thus, increase identity conflict and tension (Stroink \& Lalonde, 2009). What is missing from these discussions, however, is the role of power relations in mediating the contexts where racialized individuals "choose" between their heritage and mainstream identities. Furthermore, this review revealed the paucity of research exploring the perspectives of young Asian women and their mental health resiliencies. Current literature on Asian youth's mental health focus mostly on their problems and challenges, with little emphasis on their resilience and experiences of agency and the conditions (or constraints) that shape such outcomes.

\subsection{Gaps in Literature}

While researchers have advanced current knowledge on Asian individuals' acculturation/enculturation and mental well-being in North America, it presents an incomplete 
picture of the phenomena. Firstly, most of the relevant themes from the literature were yielded from American studies, which differ from Canada in immigration, political, and healthcare climates. Secondly, studies were primarily quantitative in nature, utilizing surveys and scales to measure and establish a relationship between acculturation/enculturation, generational status, and mental health outcomes among Asian individuals. There were no studies using a narrative approach. The quantitative nature of these studies does not offer a nuanced understanding of broader contexts and discourses that shaped the findings. Further, these results offer a dichotomous perspective that perpetuate racist stereotypes by ascribing mental health outcomes to "cultural" determinants.

Without contextualizing acculturation/enculturation experiences and mental health within the dominant discourses of their socio-cultural environments, we continue to perpetuate women's oppression and reinforce individual-focused recommendations. Lastly, studies included in this review lacked critical theoretical frameworks that could offer knowledge and important insight beyond acculturation/enculturation theories. Without the foregrounding of social justice theories (namely, critical race and critical feminist theories), we continue to ignore how larger structures of power relations, racism, and sexism simultaneously operate to produce inequities or opportunities that shape young Asian women's mental health outcomes. This thesis addresses several gaps in literature by incorporating critical narrative analysis (CNA), critical race theory (CRT), and critical feminist theory (CFT) in the exploration of young Asian women's growing up and mental health experiences (see Chapter 3).

\subsection{Summary}

In this chapter, I presented a review of existing evidence on young Asian women's racialized-gendered growing-up experiences, identity construction, and mental well-being. This 
included a critical analysis of current discourse and existing gaps in research on "Canadian" and "Asian" cultures and identities. While scholars have achieved theoretical advancements in exploring acculturation and enculturation experiences of young Asian women in North America, many inconsistencies exist. What remains critical is the acknowledgement and interrogation of how broader social structures and power relations operate within young Asian women's "acculturation" and "enculturation" experiences, which in turn shape young Asian women's identities and mental well-being. In Chapter 3, I describe the theoretical and methodological orientation used to address these gaps. 


\section{Chapter 3: Methodology}

\subsection{Introduction}

This chapter presents an overview of the theoretical framework and methodological approach for this study. A discussion of critical race and critical feminist theories and their relevance to acculturation and enculturation concepts is provided. This chapter also presents narrative research and critical narrative analysis as my methodological approach to explore my research questions.

\subsection{Theoretical Frameworks}

\subsubsection{Critical Race and Feminist Theories}

This narrative research drew on critical race and critical feminist theories to interpret and explore my research questions. Critical Theory aims to expose oppressive relationships within groups, challenge assumptions in which structures of race, class or gender are situated, and ultimately, empower individuals to transcend constraints imposed by dominant discourse (Creswell, 2013). Drawn from Critical Theory, CFT examines the ways in which patriarchal norms reinforce or undermine the oppression in women's economic, political, and social realities (Tyson, 2014). While women's stories, perspectives and voices are of central importance, CFT analyzes these in light of the social and historical environments in which they occur (Creswell, 2013). Additionally, CFT promotes research that is transformative, advocating for political and social action that challenges existing hegemonic gender ideologies (Creswell, 2013). When exploring Asian women's growing up experiences and perspectives, it is imperative to also examine their multiple cultural, gender, and power positioning, and how these locate them at various levels of oppression (Samuel, 2010). To this end, CRT was used as an orienting lens within the study. CRT recognizes race as a social construct and examines ways in which racism 
is embedded in social institutions, focusing on the stories of discrimination and racism from the perspective and experience of people of colour (Creswell, 2013).

A key tenet of CRT is to advocate against racial inequalities (Creswell, 2013) and societal claims of objectivity and neutrality (Teranishi, Behringer, Grey \& Parker, 2009). This research was conducted with CRT and CFT in the foreground of all aspects of the research process. These theories acknowledge the subjectivities and powerful positions of the research team and challenge them to reflect on their own assumptions and interpretations (Madison, 2005). Specifically, these theories underpinned the data collection and analysis processes, recognizing the participants' individual complex identities and contexts to ensure that the study did not further marginalize them (Creswell, 2013).

Racialized women face the expectations of meeting both hegemonic gender and racialized ethnic standards venerated across the mainstream and their heritage cultures (Pyke \& Johnson, 2003). This may enable or constrict their mental health. Results of the literature review showed that researchers frequently operationalize conventional acculturation theories. Very few used enculturation theories. Therefore, in the next subsection, I offer a critical analysis of current discourses of acculturation and enculturation theories while integrating CFT and CRT.

\subsubsection{Acculturation and Enculturation Theories}

Conceived in the field of anthropology, early theories conceptualized acculturation as a unidimensional/unidirectional process in which immigrants adapt and internalize the new, dominant mainstream culture (Gordon, 1964). This model suggests that identity and cultural changes occur on a single continuum. As individuals adjust to the mainstream culture, they inevitably discard their ties to their original (heritage) culture (Schwartz, Unger, Zamboanga, \& Szapocznik, 2010). Therefore, the only possible outcome for unidirectional acculturation is 
assimilation (Flannery, Reise, \& Yu, 2001). From the CRT and CFT perspective, this approach is pervasively oppressive and embodies much of the deliberate colonial ideology produced by Western norms (Ngo, 2008). When social and health disparities are explained as the "failure" of individuals or groups to acculturate, it perpetuates the notion of Otherness and conceal the invisible roles of unequal power, marginalization and oppression of Whiteness and racism (Ngo, 2008). To address criticisms of unidirectional acculturation, a bidimensional/bilinear approach was developed. Within this school of thought, Berry's $(1974,1980)$ bidimensional theory is considered one of the most prominent and influential theoretical frameworks evidenced by its widespread use in cross-cultural psychology (Miller, 2007). Therefore, it was explored and critiqued as the main frame of reference in this study.

Berry proposes two main dimensions underlying the acculturation process of immigrants: cultural adaptation and cultural maintenance. Cultural adaptation refers to the adaption of new values and practices from the mainstream culture, whereas cultural maintenance is the adherence to one's heritage culture (Berry, 1980). Berry suggests that individuals may be simultaneously oriented to the mainstream and heritage culture, resulting in four possible acculturation strategies: assimilation, marginalization, separation and integration. Assimilation occurs when the individual becomes "highly acculturated" to the mainstream culture, embodies the dominant culture, and no longer identifies with his/her heritage culture (Sam \& Berry, 2006). Marginalization is located on the other end of the spectrum, wherein neither the mainstream or heritage culture is maintained (Sam \& Berry, 2006). Due to the lack of participation in both cultures and its potentially isolating nature, this acculturation strategy has been associated with negative health outcomes (Ruzek et al., 2011). Separation occurs when the individual maintains a high level of adherence to their heritage culture and "low acculturation" to the mainstream 
culture (Sam \& Berry, 2006). Lastly, integration is present when the individual simultaneously participates and adheres to the mainstream and their heritage cultures (Sam \& Berry, 2006). This strategy proposes that it is possible for individuals to participate in both cultures without compromising their sense of cultural identity - a phenomenon commonly referred to as biculturalism (Berry, 1980; Kim \& Omizo, 2010).

Some researchers suggest that biculturalism is associated with favourable mental health outcomes, especially among young immigrants (Schwartz et al., 2010). It is important to note that Berry's theory implies the notion of choice among marginalized individuals and their ability to choose their acculturation strategy (Berry, 1997). In addition, acculturation changes are not limited to a "cultural" dimension; they may have psychological (emotional, cognitive) and sociocultural (practices and values identifying with specific cultural milieu) dimensions (Sam \& Berry, 2006).

While the concept of "acculturation" has dominated research on diaspora and migration, some authors argue that it may not comprehensively encompass the experience of individuals born into the dominant culture, some of whom may not be fully enculturated into their Asian culture (Kim et al., 2009). Kim (2007) proposes “enculturation” as a more representative term for this phenomenon and defines enculturation as the "process of (re)learning and maintaining the norms of the indigenous culture" (Kim et al., 2009, p. 27). Incorporating enculturation in research promotes an equal level of importance to the concept of learning and maintaining one's heritage norms as much as acculturation (Kim, 2007). Furthermore, this concept emphasizes human agency, i.e., individuals are not passive recipients of dominant "culture" as implied by traditional acculturation theories (Weinreich, 2009). Guided by CRT and CFT, I critically 
analyzed young Asian women's experience of acculturation and enculturation and their influence on their mental health outcomes.

Although Berry's bidimensional approach may be used to explain certain cultural adaptation phenomena, the lack of consideration of power relations and structural inequality in this approach make invisible the systemic barriers encountered by racialized immigrants and families throughout the integration process. This approach allows acculturation researchers to imply and justify "assimilation" as an acceptable and desired practice if the racialized Other wish to successfully integrate into the dominant Western culture (Viruell-Fuentes et al., 2012; Weinreich, 2009). Acculturation is frequently reduced to a binary process that ascribes mental health outcomes to one's acculturation strategy. For example, it is suggested that Asian individuals who are "highly acculturated" or "assimilated" are more likely to experience positive mental health compared to their highly enculturated counterparts (Yoon et al., 2017). This approach negates the multiplicity of historical, political, social, and economical domains that are inextricably interwoven in their lives, and how these forces influence the social integration of visible minorities (Viruell-Fuentes et al., 2012; Chirkov, 2009b). Another core criticism of bidimensional acculturation lies within its seemingly static and structural classification system (Yoon et al., 2017). It fosters a "one size fits all” approach that characterizes all migrants equally, regardless of their contexts or ethnic backgrounds (Schwartz et al., 2010). Without integrating theoretical frameworks such as CFT and CRT, acculturation studies may promote the myth of homogeneity among ethnic groups and consequently reinforce cultural stereotypes (Gupta et al., 2013).

A bidimensional acculturation approach also minimizes other factors of the acculturation experience. Everyday individual and collective experiences of racialization, gender 
discrimination, and oppression shape the (re)construction of multiple identities within their mainstream and heritage spaces (Bhatia \& Ram, 2009). Therefore, it can be argued that acculturation is a fluid process that evolves over time. It is not dictated by fixed measures such as generational status, native language, citizenship, or age of migration (Viruell-Fuentes et al., 2012). Throughout their lives, 1.5 and 2.0 young generation Asian women continuously interact with dominant discourses, navigating and negotiating circumstances that may be imposed on them based on their gender, race, and class. Their sense of identity and social integration will vary over time, which may include a false sense of self that is perpetuated by internalized racial and gender oppressions (Ngo, 2008). Hence, acculturation theory as defined by Berry may not provide a holistic explanation of young Asian women's growing up and mental health experiences.

In addition, a bidimensional acculturation approach detracts attention from the broader, structural factors to perpetuate the notion of "choice" and victim-blaming, suggesting that "acculturation outcomes" are determined by the acculturating individual rather than their interactions with the dominant structures and group. By implying that individuals choose to be assimilated, separated, marginalized or integrated, we assume horizontal positioning of both heritage and mainstream cultures (Bhatia \& Ram, 2009; Schwartz et al., 2010). This notion perpetuates claims of neutrality and equal opportunity and promotes the model minority myth frequently associated with Asian communities (Ngo, 2008). Without foregrounding acculturation within CRT and CFT, we risk obscuring the role of exclusionary immigration laws, inequitable labour policies, biased gender expectations, and social hierarchies that (re)produce oppressions among young Asian women. 
Nonetheless, it is possible that some visible minorities choose certain dimensions of their acculturation and enculturation narratives. We are not passive recipients of experience. In fact, I would argue that as human beings, we continuously engage in producing, perpetuating, resisting, and/or challenging dominant discourses that influence our actions. We have the capacity to exercise our agency (within constraints) as we engage in ongoing negotiations and construct our own interpretations of dominant discourse (Chirkov, 2009b). For example, immigrants who are fluent English-speakers may still choose to use their heritage language because of cultural pride and/or as a deliberate tool of resistance against racial discrimination (Viruell-Fuentes et al., 2012). Cultural diasporas are formed when immigrant communities cannot find themselves represented within the mainstream culture and in turn, provide sources of protection and belonging (Bhatia \& Ram, 2009). Acculturation, then, must involve some deliberate reflection and action on behalf of the individual during the process of meaning-making across heritage and mainstream communities; a "dynamic, back-and forth, play concurrently between structure and self” (Bhatia \& Ram, 2009, p. 147). The conditions produced by broader gender and racial landscapes as well as the young Asian women's agentic identities simultaneously shape their growing up experiences. Therefore, they cannot be analyzed independently. The integration of CRT and CFT to acculturation theories provides a more nuanced approach to exploring their growing up and mental health outcomes.

\subsection{Design and Method}

\subsubsection{Narrative Research}

Researchers use the terms "stories" and "narratives" synonymously, and will henceforth be referred to as such. Individuals construct narratives as a way of organizing interpretations of their past and present experiences and future realities. Embedded within these stories are personal 
reflections, judgements, and assessments that may represent our understanding of our lifeworld and how we claim our self-identities (Reissman, 1993). While narratives are linked to the subjective experience of the individual, they are also bound to meso- and macro- discourses that construct such experiences. Specifically, the stories young Asian women shared about their growing up and self-identities are inextricably shaped by racialized and gendered societal constructs.

Individuals share narratives regularly to perform a variety of functions such as providing information, communicating thoughts and feelings, or expressing actions and experiences (Holloway \& Freshwater, 2007a). We particularly narrativize experiences that present a "breach between ideal and real, self and society" (Reissman, 1993, p. 3) and thus, it is a means to make sense of self and reality. These narratives embody our interpretation of the experience. Although they may not be a total representation of the event, they offer pathways to broaden our understanding of certain phenomena. Reissman (1993) argues that narrators are socialized not to critically reflect on their experiences with racial and gender inequality. Therefore, in studying narratives, we do not only focus on its content, but also how it was shared. Considered as a whole, other narrative properties such as sequence, tone, context, and function may broaden our ability to understand lived experiences (Wells, 2011) and therefore, provide meaningful opportunities for individual and collective empowerment - a key principle of nursing practice.

To explore the growing up experiences and mental health of young Asian women in the broader context of culture, enculturation, and acculturation, I used the narrative approach. Narrative research gives prominence to individual subjectivity and identity, while facilitating a deeper understanding of experiences with marginalization and oppression (Holloway \& Freshwater, 2007b). This research approach is closely aligned with the study's theoretical lens, 
CRT and CFT, which were used to make sense of the participants' narratives. Narrative research does not seek to produce generalizable truths, but rather, it embraces the ontological belief that multiple realities exist and are unique to each individual (Creswell, 2013). This approach enables the researcher to explore how each participant constructs and performs their identities and femininities within her own sociocultural contexts. Ultimately, listening to and analyzing participants' stories invites a critical examination of their lived experiences, which may surface underlying and oppressive discourses. It is an approach that regards the participants as the experts with insightful experiences, urging researchers to look deeper and beyond normalized discourses in which such structures are embedded.

Narrative research also supports the epistemological assumption underlying all qualitative studies, which values the subjective interpretation of experience and knowledge of each individual (Creswell, 2013). It is within this approach that researchers can listen to gain deeper understanding of the experiences of acculturation and enculturation, and how 1.5 and 2.0 generation young Asian women construct their identities, growing up experiences, and mental health in Canada. It respects participants' subjectivities about "being” a young Asian woman, recognizing their agency and power to control their own story (Holloway \& Freshwater, 2007b).

\subsection{Recruitment and Study Participants}

The original CIV study team consisted of a nominated principal investigator (Dr.

Josephine P. Wong), one graduate research associate, one undergraduate coordinator and four undergraduate research assistants. Upon receiving approval from the Ryerson Research Ethics Board (REB 2012-259), the study team employed multiple strategies to recruit participants, including recruitment through networks of personal and community services organizations, student associations, utilizing both hard copy and electronic flyers. Recruitment flyers described 
the focus of the study (exploring young Asian women's growing up experiences, identities, sexual and emotional health) and encouraged interested participants to contact the coordinator.

Since the CIV study explored the phenomenon of acculturation and identity construction, 1.5 and 2.0 generation individuals were recruited. Participation criteria were: youth between the ages of 18 to 23, from East, South, and/or South East Asian backgrounds, born in Canada, or have lived in Canada for more than 7 years, residing in Toronto. A team of five young Asian women were hired as research assistants to facilitate the data collection process and engage in a deeper exploration of their narratives and the phenomena (Creswell, 2013). A total of 14 selfidentified young Asian women were recruited to take part in the study. Additional demographic data is outlined in Table 1.

Table 1: Participant socio-demographic data

\begin{tabular}{|l|l|l|}
\hline Ethnic Background & $\#$ & Ages \\
\hline Chinese & 3 & $21,22,22$ \\
\hline Filipino & 2 & 19,21 \\
\hline Indian & 2 & 21,23 \\
\hline Korean & 2 & 21,22 \\
\hline Pakistani & 1 & 19 \\
\hline Sri Lankan & 3 & $22,22,23$ \\
\hline Vietnamese & 1 & 18 \\
\hline Generational Status & \multicolumn{2}{|l}{} \\
\hline 1.5 Generation & 5 & \\
& & \\
\hline 2.0 (Second) Generation & 9 &
\end{tabular}

\subsection{Data Collection}

After obtaining informed consent, the participants were engaged in a series of three semistructured focus group interviews, each lasting up to 2.5 hours in length, and taking place at least one week apart. The participants were divided into three groups - Group $1(n=4)$, Group $2(n=6)$, and Group $3(n=4)$. The group interviews focused on their growing up experiences, identity construction, perspectives on culture, gender, sexuality, emotional health, and their individual 
and collective resiliencies. To protect the confidentiality and safety of the participants, questions about personal culture, self-identity, sexual values and practices were only asked within the preinterview surveys, which were completed in privacy.

Each focus group was facilitated by Dr. Wong and two research assistants (RA). In addition to the focus groups, nine participants volunteered to take part in individual in-depth interviews. These individual interviews were conducted by Dr. Wong. This secondary data analysis study drew on the data collected through focus groups and individual interviews.

During the data collection stage, of which I was an active co-facilitator, we used semistructured interview guides, comprised of open-ended questions and probes, to facilitate focused discussion across all participant groups. The open-ended nature of semi-structured interviews encouraged participants to share their perspectives and experiences (Creswell, 2013). Central to the interview was the flow of storytelling, facilitated by minimizing unnecessary questions (Holloway \& Freshwater, 2007b). This method was appropriate for the study because it enabled the study team to draw from the participants' rich stories to understand their acculturation and enculturation experiences. As the interviewers, we played an active role in co-creating the narratives through probing, clarifying, omitting, or restructuring questions but most importantly, through active listening (Holloway \& Freshwater, 2007b). The interview guide allowed for a semi-focused discussion and the study team engaged in flexible, critical listening to clarify or ask participants to elaborate on experiences that were not outlined in the guide.

All focus groups and individual interviews were conducted in a private meeting room at Ryerson University and audio-recorded. Digital audio recordings were transcribed verbatim, deidentified, and uploaded onto an encrypted, password-protected computer at Dr. Wong's office. Transcripts were checked for accuracy by two team members. The computer is located in a 
locked, secure office that is accessible only to Dr. Wong and CIV team members who had signed confidentiality agreements.

\subsection{Data Analysis and Interpretation}

For the purposes of this thesis, I engaged in secondary data analysis of the focus group and individual interviews and focused on the growing up experience and mental well-being of the participants. I used N-Vivo to manage the data. To develop a list of thematic codes for use with N-Vivo, I undertook a number of processes: (1) familiarized myself with participants' narratives by listening to the group and individual interviews and reading the transcripts; (2) produced a 2-page summary of each transcript to ensure the contexts of participants' narratives were not lost through coding (Wong, 2014); (3) developed a list of thematic codes with definitions; and (4) applied the thematic codes to transcripts using N-Vivo. The code book was reviewed and agreed by Dr. Wong and the Thesis Committee.

My data analysis and interpretation were informed by CNA as developed by Langdridge (2007). CNA combines critical discourse and narrative analysis in a mutually informing framework that aims to explore how personal narratives and institutional discourses influence one another (Souto-Manning, 2014; Chouliaraki \& Fairclough, 1999). On its own, narrative analysis is concerned with the human experience and how we espouse meaning and value to these experiences through language (Souto-Manning, 2014). On the other hand, critical discourse analysis examines discursive ideologies that have been normalized in everyday narratives over a period of time, and the maintenance of power differences through the use of language in institutional contexts (Souto-Manning, 2014).

CNA simultaneously and systematically considers the micro- and macro-contexts within which humans construct and present their narratives. Personal narratives are experienced and 
situated within social, power, and institutional discourses. As such, they must be critically analyzed and deconstructed within these contexts. CNA affords an exploration into how institutional discourses assert their power into the everyday experiences of the participants (Souto-Manning, 2014) - which are key tenets of CRT and CFT. CNA problematizes the neutralization of institutional discourse and shifts the power of discursive practice to increase individual agency and collective capacity for committed change (Souto-Manning, 2014). Asking questions, deconstructing and re-framing narratives within larger institutional discourses can foster critical meta-awareness and engage individuals in transformative social and political action against oppression (Freire, 1970). Thus, CNA can be a resource to unveil and challenge gendered and racial constructs imposed on young Asian women's narratives and encourage agency.

The six stages of Langdridge's (2007) CNA framework informed data analysis and interpretation. The stages include the following:

A critique of the illusions of the subject. This first stage urges the researcher to engage in reflexivity by reflecting on their personal values, experience, and background in relation to the topic of inquiry, and the impact these bear on conduct of the study (Langdridge, 2007). Langdridge (2007) suggests reading through the entire study transcript with openness to become familiar with the nuances and meanings of the issue(s). I engaged in reflexive writing to reflect on how I resonated with the data, including my personal beliefs and contextual experiences that may have shaped my perspectives as a 1.5 generation young Asian woman. This stage aims to elicit awareness of my own lifeworld, such that I also construct my narratives within sociocultural discourses that both allow and limit my own way of thinking and being (Langdridge, 2007). 
Identifying narratives, tone and rhetorical function. The second stage includes a review of the transcript to identify and delineate distinct narrative(s) in relation to the research aims (Langdridge, 2007). This also includes an examination of the tone and function of the stories. Identifying the tone may provide deeper insight into messages that may not be apparent in the content of the narrative. In addition, narratives and dialogue serve a function - to respond to other messages (Bakhtin, 1986 in Langdridge, 2007). Thus, I asked questions such as: What is the story being shared? What is the function of the story? What is the conversational context in which the story was produced?

Identities and identity work. The third stage explores the self-identities that participants construct and present through their narratives. I asked questions such as: Who is this participant? How does this narrative portray the participant in relation to what I know about her (age, ethnicity, and gender)? This stage intertwines with and complements the second stage.

Thematic priorities and relationships. The fourth stage requires the researcher to re-read the text to "identify the themes and relationships between the themes in the story" (Langdridge, 2007, p. 138). While deriving the major themes in the text, the broader contexts of the narrative(s) were maintained by referring back to the transcript summaries and notes. I engaged in inductive and deductive thinking to record and organize the themes according to (1) what the participants articulate, and (2) the research aims and theoretical framework of the study.

Destabilizing the narrative. The fifth stage includes the application of CRT and CFT as an integrated lens to review and interrogate the data in terms of participants' social positioning and contexts related to race, gender, age, class, and sexuality of the participants. 
Critical synthesis. The sixth and final stage presents the analytical synthesis of findings based on the CNA framework. I collaborated with my thesis supervisor and committee members to explore, dialogue, and question the data to reach the interpretations (Stacey et al., 2016).

Adopting CNA allowed me to analyze the participants' growing up and mental wellbeing narratives within the context of societal discourses of power, culture, acculturation, and enculturation. As I analyzed the data, I critically examined how dominant structures and institutions (that are frequently accepted as neutral and universal) function to oppress and marginalize those who do not "fit" into White Canadian and North American ideals. I also explored the social and historical contexts within which these narratives occur, and how they influence meaning-making and interpretation among the participants. The components of this approach complemented and encompassed the central principles of CRT and CFT, thus ensuring that the work was grounded in both theories.

\subsection{Establishing Rigour}

Evaluating the quality of narrative research cannot be reduced to traditional notions of reliability and validity. Narrativization serves a certain purpose and assumes a specific point of view, depending on the values and perspectives of the narrator (Reissman, 2008). Individuals are implicated in evolving social discourses and power relations and so, it is possible for the same narrative to be shared in different ways (Reissman, 2008). Since interpretive narrative research is interested in multiple was of knowing and depth of experience rather than one objective "truth", validation strategies must be reconceptualized into reliability (Mischler, 1990).

I followed Reissman's (2008) three suggested approaches for reliability in narrative research: (1) persuasiveness, (2) coherence, and (3) pragmatic use. Persuasiveness was strengthened through analysis of participants' narratives alongside theoretical frameworks of 
CRT and CFT. Multiple interpretations of the data were considered, analyzed, and documented in collaboration with my thesis committee. Coherence refers to the "thick" analysis of a text that occurs in a global, local, and thematic level (Agar \& Hobbs, 1982). This was ensured through continuous interrogation of participants' narratives in relation to their multiple identity positionings (global coherence), the narratives' structure in light of its intended role and effect (local coherence), and recurrent themes and underlying assumptions in regards to the topic of inquiry (thematic coherence; Reissman, 2008; Agar \& Hobbs, 1982). Pragmatic use refers to the extent to which a study can serve as a basis for future studies. While pragmatic use of this study (as defined by Reissman, 2008) cannot be ascertained due to the constant social construction and evolution of nursing research, this was maximized through transparently describing how the data was analyzed and interpreted through the development of an audit trail. Regular meetings were held with my thesis committee to dialogue about analytical themes and recorded in the form of meeting minutes.

I acknowledge that the findings I present in this thesis offer one of many ways that can be used to interpret the participants' narratives. My social positioning, personal orientation, and experiences shape my undertaking of this inquiry (Creswell \& Poth, 2018). Throughout the

process of this study, I engaged in reflexivity to facilitate dialogues between my "self-as-Krisel", and my "self-as-researcher". My reflexive process illuminated how my location shapes my understanding and interpretation of the narratives and in turn, strengthened my ability to ground my findings in the data rather than imposing my personal assumptions.

\subsection{Ethical Considerations}

As stated earlier, I was involved in the capacity of a research coordinator in the CIV research project, which received ethics approval from Ryerson's Research Ethics Board (REB 
2012-259). For this secondary analysis, I have also obtained approval (REB 2017-402) from Ryerson Research Ethics Board to conduct my analysis (see Appendix A). Since the data I used did not contain any identifiable information, no further documents were required. Data analysis was conducted as per the approved CIV protocol. I received access to the data upon the committee's approval of the thesis proposal. All data remained securely stored in a password protected computer system.

\subsection{Reflexivity and Situating the Self}

The relational nature of narrative research requires reflexivity at all points of the study. Reflexivity involves thoughtfulness and self-awareness from the researcher, consciousness of their own values, experiences, and reactions and awareness of their power and social positioning in the context of the researcher-participant relationship (Holloway \& Freshwater, 2007b). This thesis is shaped by my personal context and social positioning as a 1.5 generation young Asian immigrant, grounded in social justice principles. My individual background and experiences shaped my initial and ongoing intellectual interaction with the data and theoretical frameworks I employed. During the data collection phase of the CIV study, I strongly resonated with the participants' sentiments and narratives of their growing up experiences. My personal experience of racial and gender discrimination and my resilience as an Asian woman resonated with the participants' narratives, and this resonance fostered a sense of shared collectivity and connectedness. However, an inherent imbalance of power between the researcher and participants exists because I held the power to (re)present their stories (Muller \& Gubrium, 2016). Thus, I was also aware of my own social location and background as I took on the researcher's role to analyze their stories. 
Within the context of this secondary analysis study, I remained inextricably linked to the participants' narratives based on our similarity in age, language, and ethnic backgrounds at the time of the interviews. Critical scholarship calls researchers to be more honest and forthcoming in all aspects of the study. Rather than detaching my subjective perspectives in efforts to maintain conventional claims of "neutrality" in the scholarly voice, I acknowledged what I brought into the research process and how this impacted the work (Graham, Brown-Jeffy, Aronson, \& Stephens, 2011). I continued to practice reflexivity through writing and dialogue to ensure that the participants' narratives were privileged within the analysis (Langdridge, 2007), and to critically examine how my social positioning, values, and experience shaped my interpretation of their stories.

\subsection{Summary}

In this chapter, I described the theoretical frameworks and narrative approach that guided this study. I also outlined the methods for data collection, data analysis, and interpretation. Lastly, I explicitly presented my "self" in relation to the research and research participants. The following manuscript-style chapters present the findings based on the findings informed by the theoretical frameworks and CNA. 


\section{Chapter 4: Racialized-gendered Experiences and Mental Health Vulnerabilities of Young Asian Women}

\subsection{Introduction}

Canada is often recognized as a cultural mosaic - lauded as an example of rich diversity and inclusivity in global conversations. In 2016, one in five individuals in Canada self-identified as a visible minority, broadly defined as individuals who are "non-Caucasian in race or nonwhite in colour" (Statistics Canada, 2017), with over 48\% originating from South, East, and South-East Asian countries (Statistics Canada, 2017). Of these, over half are women who settle in large metropolitan cities. The largest share of visible minority women reside in Toronto, Ontario (Chui \& Maheux, 2011).

Mental health is a critical part of an individual's state of being that enables them to realize their potential, promotes their capacity to lead a fulfilling life, cope with everyday decisions and relationships, and contribute to their communities (World Health Organization, 2014). Mental health vulnerabilities refer to conditions that render some individuals, communities, and social groups susceptible to developing mental health challenges (World Health Organization, 2012). In recent years, it has been recognized that the mental health of racialized communities is vulnerable to the negative effects of systemic discrimination, racism, and social exclusion (Mental Health Commission of Canada, 2016; Paradies et al., 2015). In North America, women emigrated from Asia are least likely to self-report good mental health (Bushnik, 2016), use mental health services (Augsberger et al., 2015), and have higher rates of suicide (Lee et al., 2009) compared to women of other ethnic backgrounds.

Existing research on the mental health and mental health vulnerabilities of Asian women focuses mostly on first generation immigrants born outside of Canada or United States (Statistics 
Canada, 2018b). Little is known about the mental health experiences of the 1.5 generation (i.e., women immigrated as children or young adolescents and have lived in Canada for 7-10 years), and 2.0 generation (i.e., women born in Canada with at least one parent born outside of Canada; Statistics Canada, 2018b).

\subsection{Literature Review}

\subsubsection{Racism and Asian Mental Health Vulnerabilities}

Racism is a social, systemic, and institutionalized practice that privileges Whiteness, justifies the exclusion of those deemed inferior to this group and thus, perpetuates racial discrimination and inequality (Gee, Ro, Shariff-Marco, \& Chae, 2009). Whiteness is a socially and politically constructed ideology that confers unequal power and privilege based on skin colour and justifies hierarchical categorization and oppression of racialized peoples (Frye, 1983). There is substantial research evidence that indicates significant impact of racism on the mental health vulnerabilities of racialized people. In particular, chronic exposures to racism may proliferate biopsychological consequences through the dysregulation of neurobiological and cognitive functions (Berger \& Sarnyai, 2015). Increased exposure to racism (such as discrimination, historical trauma) release neurohormones that compromise the immune system's efficiency and increase risk to illnesses and disease (Williams \& Mohammed, 2013; Sue, 2010). Further, racism may produce harmful emotional (i.e. feelings of loss, humiliation, anger) and cognitive (i.e. self-stereotyping, hypervigilance, fatigue) responses (Williams \& Mohammed, 2013; Sue, 2010). A meta-analysis of 23 studies revealed that racial discrimination was significantly correlated to depression, anxiety, and greater overall psychological distress for Asian Americans (Lee \& Ahn, 2011). 
Racial oppression and stereotypes perpetuated by the dominant group can also be internalized by racialized people and communities, in inter- or intra-group contexts (Bivens, 2005; Pyke \& Dang, 2003). Years of oppression and subjugation may promote beliefs and ideas that portray their own ethnic groups as inferior to dominant Whiteness (David \& Derthick, 2013). Internalized beliefs and systemic discourses may manifest through automatic deprecating attitudes, beliefs and behaviours towards themselves and others from their own ethnic groups, and lead to negative mental health consequences (David \& Derthick, 2013). One manifestation of internalized racism in Asian communities is the concept of colonial mentality. Originally conceptualized to explore the consequence of colonization among Filipino-Americans, colonial mentality refers to the "automatic and uncritical rejection of anything Filipino, and an automatic and uncritical preference for anything American" (David \& Okazaki, 2006, p. 241). Colonial mentality promotes the "self" denigration of one's culture and in turn, upholds and reinforces Whiteness.

Although enacted racism, institutionalized discrimination, and internalized racism may manifest differently in various contexts, their impact remains a critical determinant of mental health vulnerabilities for Asian individuals and communities (Garcia, David, \& Mapaye, 2018; Liu \& Suyemoto, 2016; Syed \& Juan, 2012; Cokley, Hall-Clark \& Hicks, 2011; Pyke \& Dang, 2003). Despite this evidence, the racialized-gendered mental health experiences of young Asian women remain largely overlooked in Canadian research. The limited literature on Asian Canadians' mental health tends to offer inadequate explanations that ascribe Asian mental health vulnerabilities to "cultural determinants" isolated from mainstream "Canadian" society, and attributed to "cultural characteristics" such as familial obligation (Vu \& Rook, 2012), emotional restraint (Lipsicas \& Makinen, 2010), academic success (Tummala-Narra et al., 2016), and 
conformation to norms to maintain social harmony (Shea \& Yeh, 2008). Further, contemporary Western dominant discourses portray Asians as a "model minority", a uniformly successful and well-adjusted ethnic group who, unlike their other racialized counterparts, have overcome challenges related to racism (Tummala-Narra et al., 2016; Ho, 2014). These discourses perpetuate ethnic division and structurally reinforce stereotypes and racialized expectations. They also present an over-generalized narrative of Asian experiences, negating how systemic racism and socio-historical contexts construct "the" Asian culture to reinforce a subtle form of Othering.

\subsubsection{Sexism and Asian Women's Mental Health Vulnerabilities}

Gender discrimination, or sexism, is the use of social power to systematically subordinate women and deny access to rights, resources, and representation on the basis of their gender (Sue, 2010; McGibbon \& Etowa, 2009). This social practice continues to produce nuanced contexts for women's mental health vulnerabilities. For instance, sexism is directly correlated with poor mental health outcomes for women (Borrell et al., 2011). Power relations based on gender influence women's access to power, opportunities, and resources, which are intricately linked to mental health determinants such as income, social inclusion, sense of safety, and self-esteem (Hill \& Needham, 2013; Canadian Mental Health Association, 2008).

Research on Asian women's mental health experiences in the Canadian context are limited. This is especially concerning since Asian women in Canada are least likely to report good mental health compared to women of other ethnic backgrounds (Bushnik, 2016). Further, existing literature ascribes Asian women's poor mental health to exposure to pervasive racial stereotypes that exoticize, objectify, and sexualize Asian women. These stereotypes may be internalized and further jeopardize their mental health vulnerabilities (Yoon et al., 2017). 
Some studies suggest that Asian women who strongly identify with their ethno-cultural identities are more likely to experience depression and anxiety compared to their non-Asian counterparts. However, these studies tend to have a limited and often problematic definition of cultures. For example, Leu et al. (2011) define "traditional" cultural values as familial harmony, subservience, and modesty and imply that Asian women bear the expectation of maintaining cultural continuity regardless of the socio-environmental context. The authors fail to differentiate between "cultures" and "patriarchal practices" within cultural communities. Furthermore, these discourses of culture perpetuate the stereotypes of Asian cultures.

To understand how racialized-sexism affects Asian women's mental health vulnerabilities, critical research on the impact of broader institutions of patriarchy, discrimination, and colonialism is needed (Hewitt, 1991). However, with a few exceptions, (i.e. Mukkamala \& Suyemoto, 2018; Sue, Bucceri, Lin, Nadal, \& Torino, 2007; Pyke \& Johnson, 2003), current literature on Asian women's health tends to contextualize their experiences through a single lens of either culture, racism, or sexism. There is a paucity of research that explore how the complex intersection of race, gender, class and other markers of difference, shape the experiences and pathways of resilience specific to Asian women's mental health.

\subsubsection{Generational Status and Mental Health Vulnerabilities of Asian women}

Recent research shows that generational status is found to be correlated to mental health outcomes for Asian populations (Alamilla et al., 2017). First generation Asian women are more likely to have lower rates of mental health disorders compared to their 2.0 generation counterparts (Takeuchi et al., 2007). Limited literature suggests that 1.5 and 2.0 generation Asian women experience unique mental health stressors as they construct their racialized-gendered identities in the competing expectations and contexts of their ethnic and the mainstream 
"Canadian" culture (Rajiva, 2006). Some of these stressors include having to deal with pressures to conform to competing social norms and contrasting messages from their parents, communities, and the dominant White settler society (Tummala-Narra et al., 2016; Suarez-Orozco \& Qin, 2006). Specifically, Asian youth experience increased social isolation (Rhee et al., 2003), anxiety levels, and lower self-esteem compared to their non-Asian counterparts (Tumala-Narra et al., 2016).

Less is known about 1.5 and 2.0 generation Asian women's mental health resiliencies, how they cope and thrive within their mainstream and ethno-cultural contexts. As 2.0 generation individuals represent $17.7 \%$ of the Canadian population and are projected to grow steadily (Statistics Canada, 2017), the dearth of research on their mental health vulnerabilities and resiliencies is a concern. This research aims to narrow existing gaps in North American literature by further exploring the mental health vulnerabilities of 1.5 and 2.0 generation Asian women, while critically situating their narratives within the interdependent contexts of racializedgendered, systematic patriarchy, colonialism, and class-based hierarchies.

\subsection{Methodology}

This study used a narrative research approach to explore the racialized-gendered experiences of young Asian women. Narrative research systematically explores how individuals interpret their worldviews and the broader socio-historical contexts that shape these narratives. Explicit in this approach is the assumption that narratives reflect our understanding, perspectives, judgments, and reflections about our life worlds and self-identities (Reissman, 1993). At the same time, our narratives are also informed by dominant and resistant discourses (re)produced through power relations in society. In particular, growing-up stories shared by the young Asian women are inextricably shaped by discourses of race, gender, class, and migration. As we 
explored the participants' experiences, we were also concerned with how they interpreted and ascribed meaning to them within larger systemic structures (Reissman, 1993), and how these influenced their mental health vulnerabilities.

This approach is aligned with the study's theoretical frameworks of intersectionality, critical race and feminist theories (CRT and CFT). CRT and CFT analyzes the oppressive, culturally and historically contingent power relationships that undermine racialized women's realities (Creswell, 2013). Intersectionality interrogates how systems of race, gender, and class mutually produce conditions of oppression or resistance for social groups (Cole, 2009). Particularly, the concept of racialized-sexism is especially important to the study. Racializedsexism (or gendered-racism) refer to the simultaneous experience of racism through gender, and experience of gender through racism (Essed, 1990). Although it is important to note that racialized-sexism produce varied contexts for different groups of racialized women, this framework is a critical entry point into a nuanced discussion of how racism shape discourses of Asian femininity (Pyke \& Johnson, 2003) and how manifestations of gender are shaped by experiences of racism (Matthews, 2002). Without nuanced consideration, we risk oversimplifying Asian women's racialized-gendered experiences by assuming they share similar contexts with Asian men and White women (Mukkamala \& Suyemoto, 2018), further reinforcing oppression and systemic Whiteness.

\subsubsection{Recruitment}

Prior to outreach and recruitment, the research team has received approval from the Ryerson University Research Ethics Board (REB). Participants within the Greater Toronto Area were recruited in-person or online through electronic flyers, networks of personal and/or community-based organizations, student associations, and on university campus. Recruitment 
materials outlined the topic of inquiry (growing up experiences and cultural identities of young Asian women) and encouraged interested participants to share their narratives in a small focus group setting by contacting the project coordinator through email or phone. Participation criteria included: youth aged 18 to 23, identified as East, South, and/or South East Asian, who were either born in Canada or have immigrated to Canada for more than seven years and settled in Toronto.

\subsubsection{Participants}

A total of 14 self-identified young Asian women between the ages 18 to 23 years old participated in the study. The participants originated from seven different countries within the Asian continent. Nine participants identified as 2.0 generation individuals born in Canada to at least one first generation parent. Five participants identified as 1.5 generation individuals born in their heritage countries and immigrated to Canada ten years prior to joining the study. Most of the participants ( $\mathrm{n}=11)$ obtained their primary education in Canada. At the time of the study, the participants were either attending high school $(n=1)$, college or university $(n=8)$, working in their field $(\mathrm{n}=2)$, or graduated and were applying to law school $(\mathrm{n}=1)$. The average income of participants' neighbourhoods varied from low $(\mathrm{n}=1)$, middle-low $(\mathrm{n}=2)$, middle $(\mathrm{n}=6)$, and high $(\mathrm{n}=2)$, with one participant who lived on university campus.

\subsubsection{Data Collection}

All participants provided written informed consent. They were divided into three groups (Group $1 \mathrm{n}=4$; Group $2 \mathrm{n}=6$; Group $3 \mathrm{n}=4$ ) based on their availabilities, and invited to take part in a series of three semi-structured group interviews that occurred about one week apart. Twelve of the 14 participants remained in the study and took part in all three group interviews; two did not return due to time constraints. The semi-structured group interviews were dialogical in nature 
and engaged participants in critical reflection. The first group interview focused on the growing up experiences of the participants at home and at school; the second group interview explored the participants' racialized and gendered experiences in constructing their identities; and the third group interview invited participants to express their vision of an equitable social world for Asian and other racialized young women.

The group interviews were facilitated by at least two team members. Each group interview lasted about 2.5 hours. In addition, nine participants chose to take part in a semi-structured individual interview two weeks after the group interviews were completed. Individual interviews were conducted to gain deeper insight into participants' growing up experiences. All participants received the study information, a copy of their signed consent form, and a list of relevant resources and community organizations for mental health support. They also received a \$20 CAD honorarium and two transportation fares as a token of appreciation of their time and contributions. With the permission of the participants, all interviews were audio recorded.

\subsubsection{Data Analysis}

The audio recorded interviews were de-identified and transcribed verbatim. The transcripts were reviewed by at least two team members. Critical Narrative Analysis (CNA) was used to analyze and interpret the data. CNA merges narrative analysis and critical discourse in a mutually-informing framework that contextualizes micro-and macro- systems in which we experience, interpret, and present our narratives (Souto-Manning, 2014). N-Vivo 11 was used to organize the data. Study themes were inductively derived through: (1) repeated listening to the interviews and reading of transcripts to become familiar with the narratives; (2) producing a 2page summary of each transcript to ensure that the contexts and nuances of the participants' narratives were not lost through disaggregation and coding of the data (Wong, 2014); (3) 
developing a codebook with themes and definitions reviewed and agreed by at least 2 team members ; and (4) detailed line by line coding of the transcripts and reaggregating the data into distinct themes. All authors engaged in critical discussions on the thematic data analysis and the application of intersectionality, CRT, and CFT in data interpretations.

\subsection{Results}

Analysis of the participants' narratives revealed four key themes: racialized and gendered growing-up experiences, experiences of enacted racism and sexism, perceptions of familial expectations, and coping, resistance and mental health. All names in this section are pseudonyms generated by the study team to protect participants' identity.

\subsubsection{Racialized and gendered growing-up experiences}

In sharing their growing up narratives, the young Asian women shared how their growing up experiences were racialized and gendered within the mainstream "Canadian" society. One of the recurrent themes emerged from the participants' stories was bodily abjection and subjugation. For instance, Yun-Hee talked about how the mainstream media affected her perceptions of society's bodily expectations on young women:

"I had eating problems as well. I read a lot of teen magazines, like Teen People and Seventeen, and the advertisements or even the healthy sections they had would be about workouts. I did this thing where I would eat a piece of fruit for breakfast and would throw up my lunch every day. By dinner time I would be so hungry and I'd have to eat because of my family. I was going through puberty and my body was changing. But I thought any weight gain was bad. That was the message I got from reading those magazines," (YunHee, 22, Korean, 1.5 generation). 
Some participants grappled with insecurities about physical attributes that they believed deviated from the norm. Their sense of insecurity was compounded by perceived discrimination:

"People wouldn't know just by looking at me that I have hypothyroidism, but seeing that I'm larger than an average Canadian, they would judge me... On top of that, being brown, so you look different... So I think at the point where my body accumulated with my personality, which I started to doubt. At points like that, with my appearance, when they all accumulated into bad stuff, that's when I felt quite disempowered," (Sabrina, 21, Indian, 1.5 generation).

Most of the participants indicated that they had received strong messages about the desirable "norms" and White standard of beauty. At the same time, when they tried to fit into these norms, they experienced a different set of challenges, as Hanh-Ly described in her frustrations:

"For me, around the age when I was starting to use make-up, you would see all these things in magazines on how to do eye make-up, but that's for white girls with big eyes. I would be with my friends who looked so good with the make-up they tried on, and it just didn't work for me. So I just gave up on that," (18, Vietnamese, 2.0 generation).

Growing up within the context of dominant Whiteness, both as an ideology and practice, some participants shared that they received confusing messages about their body, particularly about their skin colour:

“There's White people in my school, in my elementary. They'd be like, 'Oh my gosh, I wish I was your colour!' Then there are Asians that are like, when we grew up and were in high school, that's when I started being really light, they were like, 'Oh my gosh, I really want to be your colour!' It was really weird," (Rubylyn, 21, Filipino, 2.0 generation). 
For some participants, the confusing messages about skin colour were reinforced by adult women in their family, who discouraged them from spending time outdoors in the sun and provided them with skin-lightening products or "healthy skin food." However, some participants also recalled their parents' speaking out against their relatives who encouraged them to use skin lightening products, or encouraged them to play sports and enjoy the outdoors.

\subsubsection{Experiences of Enacted Racism and Sexism}

In addition to the powerful messages of Whiteness in society, many participants spoke about their experiences of racism at the interpersonal level. For instance, Yun-Hee shared, "One time at school, people said my lunch bag smelled like Chinatown. I cried at school and then my parents went to talk to teacher," (22, Korean 1.5 generation). She went on to share that this type of marginalization continued in university. Despite her proficiency in English, one of the teaching assistants assumed that she was an ESL (English as a second language) student because of her non-Anglo name and suggested that she needed to seek help at the writing centre.

Other participants shared their experiences of racism that were much more implicit. Rubylyn (21, Filipino, 2.0 generation) recalled that her elementary school teacher discouraged her parents from teaching her how to speak Tagalog as a way to ensure her acculturation. Dalila encountered strangers asking her incredulous questions:

"I've had like weirdos come up to me before and be like 'Oh my god, where are you from?' 'Sri Lanka' 'Where is that?'... I'm not this object. Don't objectify me... I get so offended. It gets under my skin,"(23, Tamil, 2.0 generation).

Min-Jung, an architecture student in university, spoke about gender biases in her field:

"If a construction mentor comes to our school to talk about a project, then he'll automatically gravitate towards the males... It's very hard as a female construction worker 
to get a job. There is that stereotype about women being weaker than males," (21, Korean, 2.0 generation).

In addition to their own personal experiences of racism and sexism, some participants spoke of the challenges of witnessing their families' experience of marginalization post-migration. Sabrina described her parents' life back in India:

"My mom was a secretary at a hospital, and my dad had like this high position... Looking back he feels that's such a great moment in his life... then when we came here it was like... we weren't in the same kind of level economically," (21, Indian, 1.5 generation). Sabrina suggested that the decline in social status experienced by her family during migration and settlement had contributed to her father's mental health problems.

\subsubsection{Perceptions of Familial Expectations}

In the group interviews, participants spoke about their gendered experiences within their families and "cultural" communities. Some of them perceived their families' expectations on them to be restrictive or gender-biased, particularly in terms of curfews, sleep-overs, fashion, hobbies, or romantic relationships, in comparison to their non-Asian or Asian male counterparts. “They (males) just get different privileges in general... you notice the male cousins or whatever were allowed to stay out longer, or go farther up a tree when they are climbing it or go farther down the block, like all these different things that kind of make it seem like we are unequal," (Meniza, 19, Filipino, 1.5 generation).

The imposed rule that "women must be protected" resonated among many participants. They recalled dominant discourses on emphasized femininity, domesticity, family-centeredness, sexual abstinence, and/or expectations of marriage. 
"In my family... my dad is the breadwinner and my mom stopped working. She was just as educated but stopped working to raise my siblings and I. So she didn't work for twentyfour years. And I think in my mind that's something that's supposed to be a normal thing," (Kelly, 22, Chinese, 2.0 generation).

"I grew up in a pretty Filipino family. They are all Catholic so you know there is this expectation... you are supposed to save your special gift (virginity) for your special someone on your wedding day," (Meniza, 19, Filipino, 1.5 generation).

Some of the young women felt pressured to thrive in school in order to be a role model for younger siblings, gain acceptance from their parents, or "build themselves up" to become a desirable future wife. The general perception among most participants was the implicit directive to fulfill the aspirations their families had imposed on them.

It is also important to note that not all participants perceived or experienced gender biases in their families. Some of them recognized that their parents' expectations were shaped by experiences of hardship, racism and discrimination. In reflecting on her parents' migration experience, Rubylyn gained insight towards the stress and financial hardship they faced, which influenced the pressure to exceed in school:

"The security that comes from having all this education which means you have all these opportunities and there's no way that you can't have a job. And if you have a job, you can support your family, you can support yourself, you can make sure you can get all that you need. All of your needs will be fulfilled. You can pay for this, for your food, your shelter, clothing. So you'll be good. If you have education you can go a step up from all these needs. You can get better things. So I guess they want that security for you, I guess. And they left their life in another country so you could get more," (21, Filipino, 2.0 generation). 
This understanding fostered mutual respect and empathy towards her parents. It also encouraged her to become more open and communicative with them, which she identified as a source of empowerment.

Other participants referred to the support and freedom they had received from their families and communities, as Cecilia recalled:

"My parents have always wanted me to do whatever I wanted to do... they weren't very pushy about grades. When I applied to university... one of the schools I applied to was a theatre school and any other Asian parents would have been completely appalled that I was doing that but they weren't... they've always told me that I have freedom to make my own choices and stuff like that," (22, Chinese, 2.0 generation).

Other participants recalled their parents providing a counter discourse that encouraged them to pursue their own career and independence. Faye suggested that her fathers' experience of postmigration financial hardship influenced this parenting style:

"I think my father wants me to more independent. As much as he wants me to have somebody to trust and depend on, at the same time, in case anything happened, he wants me to be able to be independent and like you know, like being strong on my own," (21, Chinese, 2.0 generation).

Kameela spoke about her parents' openness in supporting her decision on how she expressed her outward symbolic of being a Muslim young woman:

"A little after I came to Canada, I started wearing the hijab which really identified me. I felt like I stuck out in a crowd, but also it gave me a strong identity... I eventually did take it off... at the beginning of grade twelve... People were like, 'Didn't your parents say anything to you about that?' And I was like, 'No, it was my choice to wear it at the 
beginning'. So, no my parents didn't say anything, they supported what I did, whatever I did," (19, Pakistani, 1.5 generation).

The participants' narratives suggested that their growing-up experiences at home and in their communities were shaped and defined by the larger social forces of sexism, racism, and practices of Whiteness in society.

\subsubsection{Coping, Resistance, and Mental Health}

The narratives of the participants suggested that their mental health vulnerabilities were intricately connected to their racialized-gendered experiences. One major theme was related to the sense of non-belonging or being in the margin within the mainstream "Canadian" context, which had shaped their vulnerability to poor mental health:

"I get really paranoid when somebody doesn't pay as much attention to me as I think they should. If a professor comes and they don't look at me while they're talking to me, I automatically think it's because I'm Asian, like she doesn't want to talk to me because I'm Asian," (Min-Jung, 21, Korean, 2.0 generation).

Another recurring theme centred on the persistent and cumulative effects of racialized-sexism and sense of compounded subordination by Whiteness and systematic patriarchy:

"I just want to add that a lot of times talking about this can be tiring. I'm tired of being a minority in a lot of ways, whether it being a woman, or an Asian person, or an environmentalist. There's this feeling that I get sometimes that I'll never be in the dominant group and knowing that is just tiring," (Yun-Hee, 22, Korean, 1.5 generation). Many participants shared that regular encounters with comments such as "where are you from?" or "tell me more about your (Asian) country" made them feel "foreign", excluded, and offended. Hanh-Ly described feelings of tension and frustration when she had to deal with such comments: 
"Sometimes I'll pump out a lecture and educate that person, but often times it's exhausting and I don't want to do that. And also because then I'd become an annoying personpreachy and overbearing. (Group laughter). So I just let things slide... I'm often put in these situations and I don't quite know how to tread the water," (18, Vietnamese, 2.0 generation).

Other participants shared responses wherein they reluctantly ceded to dominant sexist discourses. Min-Jung: "In my year, there are girls who stick together and do all their projects together but they sort of seek help from males by using their femininity to get help... Facilitator: How do you feel about that? Min-Jung: I've sort of accepted it. I guess that's one thing about women in society is that they use their sexuality to get things. And they think that it works. And honestly, I've tried to do it and it does work. I regret it after I do it because they see you as a sexual object afterwards. So you don't want to rely on that. But when push comes to shove, I'm going to do whatever it takes (to succeed)," (21, Korean, 2.0 generation).

Despite these conditions, participants offered narratives of resilience and resistance against imposed boundaries within their mainstream and heritage communities. Strategies such as joining extra-curricular groups, moving out for university, or being selective with disclosing information to their parents were employed by some of the participants to navigate their racialized-gendered everyday experiences, and build their own supportive networks, conclaves, and resources. Many openly rejected racist stereotypes that were imposed on Asian women: "Asian women are supposed to be more docile or more submissive, pale... very petite. And I don't really think I'm any of those things... so I think that came from my dad, who's like, 'play sports!',' (Kelly, 22, Chinese, 2.0 generation). 
Others engaged in sports as a protest against dominant gendered norms: "I played rugby... I beat up guys all the time... I don’t see myself as very feminine,” (Kameela, 19, Pakistani, 1.5 generation). They shared their aspirations of self-reliance and empowerment to overcome the socially imposed boundaries:

"The breadwinner is supposed to a) make more money, b) it's supposed to be the male, the woman is supposed to raise the kids. So I think that's where my empowerment comes from because I don't want to be like that. I want to be the breadwinner," (Kelly, 22, Chinese, 2.0 generation).

It is important to note that despite their strong desire to resist the dominant norms, some participants experienced constraints that limited their efforts. For example, one participant shared how the lack of resources or safe space made it challenging to deal with her depression:

"I would always like to tackle it from the source, but the source is where you live right. Honestly if I had the means, I could have left the house and live somewhere else for a while," (Faye, 21, Chinese, 2.0 generation).

For others, having economic resources did not guarantee the freedom to resist or overcome the dominant normative rules and stereotypes in their own way. Yun-Hee shared her unique struggles with math even though her parents had the financial resources to provide her with a math tutor:

“Looking back, I think I didn't do so well in calculus in grade 12 because I didn't want to fit into that stereotype of Asians being good in math. Maybe that's why I pretended I needed help in math. I don't know if I was pretending or if I didn't try hard enough. But I think I just didn't want to fit into that stereotype,” (22, Korean, 1.5 generation). 
Despite these struggles, many participants agreed that having access to education gave them opportunities to learn and challenge the dominant discourse. Dalila shared how taking a course stirred her own journey of social justice advocacy:

"I took an equity course, Intro to Equity, and my world just changed. My perspective on life, it was like my world changing moment for me. It just opened up a whole new chapter for me, I saw things so differently and so that became a major (in university)," (Dalila, 23, Tamil, 2.0 generation).

Some participants also credited their parents, partners, family members, friends, and community agencies whom served as a source of strength and confidence.

\subsection{Discussion}

Our study sought to address the existing gaps in nursing research on 1.5 and 2.0 generation young Asian women's mental health vulnerabilities by examining their racialized and gendered growing-up experiences in a White settler society such as Canada. Contrary to majority of existing research, the mental health vulnerabilities of young Asian women were not primarily shaped by "cultural" determinants such as emotional restraint, familial obligation, or academic success. Rather, data analysis revealed how sexism, racism, and Whiteness intersect to (re)produce complex experiences of marginalization specific to their socially constructed "subordinated" positions as racialized women. These imposed constructs shaped the way the participants experienced, interpreted, and performed their identities across their mainstream and ethnocultural contexts, which in turn affected their mental health vulnerabilities and outcomes.

Many of the participants referred to bodily abjections in regards to their skin colour, body size, and eye shape. The participants were encouraged to achieve a fair complexion through the use of skin lightening products or staying out of the sun. Although these messages came from 
women in their families or communities, we argue that this internalized colorism and racism is underpinned by Whiteness and a long history of colonialism around the world. Globalized Eurocentric (White) paradigms of beauty produce expectations and standards that perpetuate internalized inferiority, particularly among racialized women. The preference for light skin was a consequence of European colonialism in South and Southeast Asian countries (Ryabov, 2016). Colonialism established colour-based hierarchies that idealized Whiteness as the standard of beauty and constructed colourism, wherein dark skin was associated with unattractiveness and savagery (Chen, Yarnal, Chick, \& Jablonski, 2018). Colourism perpetuated the myth of White superiority and legitimized the subjugation of racialized and coloured peoples. Furthermore, the colonial legacy of Eurocentric supremacy and Whiteness, expressed in having light skin, is reinforced by the deep-rooted classism represented by specific markers of socially defined beauty, superiority, and refinement (Kenway \& Bullen, 2011). The cultural significance of having pale skin as a marker of privilege, desirability, and social status, elucidated by the participants' narratives, demonstrate the ongoing transnational effects of colonialism, classism, and colorism.

The participants' narratives indicated that disciplining of the participants' bodies was not limited to skin tones but also extended to other bodily ideals of Whiteness such as being blonde, thin, tall, and have big round eyes. Global capitalism and patriarchy continue to profit from subjugating racialized women's bodies to unrealistic standards of perfect beauty through commercialized enterprises of cosmetic, fashion, and aesthetic products (Alapack, 2011). Through the lens of intersectionality, these powerful normative messages shaped many young Asian women's sense of needing to alter or enhance their bodies and looks to conform or belong to the dominant environment. At the same time, as these young Asian women attempted to 
achieve the normative standard of Whiteness, they encountered a different set of challenges, that is, the beauty products in North America and Western societies are developed for White women and images of racialized women are often absent. Some participants experienced this underrepresentation as a form of environmental microaggression, that is, the "numerous demeaning and threatening social, educational, political, or economic cues that are communicated individually, institutionally, or societally to marginalized groups" (Sue, 2010, p. 25). Environmental microaggressions may lead to psychological distress when racialized young women accept dominant media images of Whiteness as the reality (Reddy-Best, Kane, Harmon, \& Gagliardi, 2018). In the context of this study, some participants struggled with their body image during puberty as they tried to fit into the normative images in mainstream teen magazines as they were constantly reminded that they were different and not measuring up to the norms. Others had to deal with the imposed 'shame' because their lunch did not fit with mainstream standard food. Furthermore, participants felt stressed and resentful towards the perpetuating stereotypes of Asian women being portrayed as exotic, hypersexual, or submissive. As reflected in other research, exposure to racial stereotypes and/or race-related bullying is associated with greater levels of body dissatisfaction among Asian women (Brady et al., 2017) and increased vulnerability to compromised mental health (Sue, 2010). For the participants in this study, this discourse modified eating practices and fostered bodily insecurities, which can have a harmful impact on mental health. Dominant gendered expectations that emphasize Western hierarchies of beauty intersect with racialized sexual objectification to produce nuanced mental health vulnerabilities for young Asian women (Brady et al., 2017).

Our findings also indicate the powerful prevalence of racial microaggressions in the young Asian women's growing up experiences. Racial microaggressions are automatic mechanisms of 
oppression that operate below the level of consciousness of individuals, promoting hostile or derogatory ideas and behaviours towards racialized individuals or groups (Sue, 2010). The impact of participants' experiences of racial microaggressions were also strengthened by discourses of patriarchy and sexism. Sexist microaggressions emerged in the participants' accounts as actual or perceived dismissal of their accomplishments, devalued contributions in academic and professional settings, sexual objectification, and denied equal access and opportunity to succeed in comparison to their male counterparts (Sue, 2010).

It is important to note that microaggressions as manifestations of racism, sexism, and patriarchy are subtle, invisible, and indirect. The "subtle" and ambiguous nature of microaggressions (racial, sexist, or environmental) may lead to internal struggles of self-doubt among Asian young women, who have to constantly question or wonder if these encounters are discriminatory or not (Sue, Capodilupo, \& Holder 2008). While the intent of the perpetrators could not be ascertained, many participants bore the emotional and cognitive labour of navigating and interpreting microaggressions in their mainstream and ethnocultural environments. They responded to microaggressions in multiple ways: from hypervigilance (constant state of questioning and anticipation of prejudicial encounters), forced compliance (conforming to White/patriarchal standards), to feeling angry, disempowered, or helpless. This emotional and mental labour is energy depleting and exhausting, and chronic exposures can lead to harmful psychological consequences and diminish quality of life (Sue, 2010).

Other participants responded to microaggressions in a manner that is misaligned with their own personal values and as such, potentially compromise their well-being. For example, one participant described using her sexuality to "get ahead" in her male-dominated profession, despite expressing her resistance against this same practice. Guided by CFT, our analysis 
suggests that the power of patriarchy is deeply interwoven into their interpersonal and institutional contexts. Although some participants voiced resistance against sexist practices in their personal and professional lives, they were implicated in upholding patriarchy as a way to compete and succeed in the White male-dominant spheres of school and work. This highlights the power of structural and systemic discrimination and the subtle, unconscious, yet persistent ways young Asian women are subjugated in preserving such inequities.

As European colonialism continued to promote White superiority throughout Asian regions, indigenous cultures were simultaneously degraded and dehumanized (Nadal, 2009). Chronic and persistent forms of racial oppression shape how the oppressed groups think about themselves and their co-ethnics, imposing racial categories that deny their right to positive selfidentity and the internalization of inferiority (Pyke \& Dang, 2003). Existing literature on Asian women's mental health vulnerabilities often negate the significant influence of these dominant norms and discourses on how participants perceived and interpreted the "cultural" expectations of their families and ethnic communities. For example, most of the participants described awareness and resistance against gendered-biases within their own ethnocultural communities. These accounts offered some insight into how these experiences were perceived as sexist by some young Asian women. However, these experiences were not conceptualized as racialized sexism structured Whiteness and racial inequity, but internalized as inferiority of the racialized communities.

Influenced by Whiteness in Canadian society, many participants perceived their parents as overly strict and their familial relationships as emotionally restrained, conformed, academically demanding, and success driven. These perceptions reflect a form of internalized racism, which perpetuate stereotypes of Asian families and Asian "cultures" (David, 2011). Internalized racism 
functions as a double-edged sword that reinforces White supremacy as racialized individuals reject their own ethnocultural communities in the hope of becoming "accepted" into the mainstream White society and share the associated privilege (Pyke \& Dang, 2003). For example, some participants shared instances when their parents, whom they described as "not your typical Asians", gave them freedom and autonomy in various aspects of family, school, and relationships. Their discourse of "non-typical" Asian parents implied that there is a generalizable standard Asian culture that imposes restrictions and oppresses young Asian women. Although most of the participants did not deliberately and overtly deny their Asian heritage, internalized racism seemed to have hooked them in such that Whiteness had become their benchmark in measuring every aspects of their life. For instance, the practice of sleepovers, which is common in the mainstream White "Canadian" culture, was used by participants as the benchmark to measure "Asian" parental control and familial restrictions. Internalized racism also perpetuates the fusion of using the idea of "culture" to explain oppressions. It takes attention away from identifying and addressing the real intersecting oppressions of racism, sexism, and patriarchy. It produces psychological distress that negatively affect Asian young women's self-concepts and well-being (Gupta, Syzmanski, \& Leong, 2011; David \& Okazaki, 2006;).

Under the lens of intersectionality, our findings also offer insight on the nuanced experience of young Asian women as they navigate the complex intersection between their gender, race, and class. For instance, some participants shared that their experiences with imposed racial stereotypes (i.e. "model minority", academic astuteness) were confounded with sexist stereotypes (i.e. exoticization, submissiveness). Within their encounters with the "model minority" myth, wherein they were assumed to be intelligent and hardworking, they also 
experienced less opportunities in their mainstream and ethnocultural contexts because of their identities as women (expectation of staying at home and raise families).

As participants shared their experiences of Whiteness through racialized-sexism in the mainstream society and throughout their heritage contexts at home, they also described a range of cognitive, emotional, and behavioural responses. Some of these responses may be considered as strategies of resilience or resistance, depending on the perceived nature of the event, social context, and/or resources available to the individual. Resilience is generally operationalized as a defense mechanism; one's attribute or capacity to thrive when faced with adversity (Davydov, Stewart, Ritchie, \& Chaudieu, 2010). By this definition, participants' narratives reflected some form of resilience when experiencing racialized-sexism. For instance, participants referred to rejecting racialized-gendered stereotypes by taking up sports, being outgoing, or requiring additional "tutoring" for math among other strategies. Although the young women voiced resistance against racist or sexist encounters, they still consciously or unconsciously reinforced these racialized-gendered stereotypes (Mukkamala \& Suyemoto, 2018). Specifically, their "rejection" of stereotypes of Asian women were associated with responses that were aligned with what is largely considered to be normative practices of White patriarchal mainstream culture. The rejection of racialized-gendered constructs still involved some aspect of rejection and conscious juxtaposition of their self-identities, thereby reinforcing Whiteness (Pyke \& Johnson, 2003). Their conditions of resistance were still dictated and implicated against larger, invisible structures of oppression. Racialized-sexism perpetuated internalized stereotypes and negatively influenced their mental health vulnerabilities, thus facilitating an uptake of behaviour that may be misaligned with their own personal values and further perpetuating discrimination. 
Other participants recognized their own internalized gendered expectations and shared their experience in resisting hegemonic discourses. For example, one participant became more engaged in her ethnic community, which strengthened her critical understanding of social justice, equity, and sense of self. Others drew on their own motivations and support from parents to pursue their career aspirations despite the existing challenges. Some participants shared how their parents, partners, and ethnocultural communities fostered a sense of self-reliance and encouraged independence. These narratives indicate how resilience is not merely an individual attribute, but is often contingent on situational conditions (i.e. interpersonal relationships, cultural and historical context, access to resources) that may be beyond their control (Davydov et al., 2010). However, the participants' experiences showed that Asian young women do have "agency" within "constraints" influenced by dominant structures of race, gender, and class.

Other participants referred to the theme of generational opportunity in the context of global migration. Witnessing the challenges and pressures faced by their parents during migration fostered a sense of suffering by association; such experiences of discrimination affected them and became internalized as their own (Viruell-Fuentes, 2007). However, this experience strengthened their understanding of their parents' perspectives, and became a source of empowerment and motivation. It highlights the diversity and heterogeneity within young Asian women and their families often neglected in current literature.

\subsection{Strengths and Limitations}

While our study makes important contributions to nursing literature on young Asian women's mental health vulnerabilities, some considerations must be kept in mind. Asians represent a heterogeneous group of individuals from diverse historical, socio-cultural, political, and economic backgrounds. The exploratory nature of a study with 14 participants, not inclusive 
of all Asian ethnicities, means that the findings may not adequately be generalizable to the target population. In addition, the study was conducted in Toronto, Canada - where $37.4 \%$ of those who immigrate to Canada choose to settle (Statistics Canada, 2018a). Our findings may not be relevant to young Asian women who reside in other provinces and face different challenges with inclusion and settlement.

All of the participants were attending or graduated from high school and university, thus limiting educational and social class diversity in the sample. Further, although focus group methodology may offer safe space to dialogue between participants and researchers, it may also influence participants' responses. While every effort was made to group participants close in age-range to minimize power differential, we were unable to successfully enact this due to participants' schedules.

To our knowledge, this is the first study that explored 1.5 and 2.0 generation young Asian women's mental health vulnerabilities in the context of intersectionality, CRT, and CFT. The findings illustrate that racialized-gendered experiences of young Asian women cannot be analyzed without critical exploration of power relations and hegemonic discourse; to do so would reproduce false culture-bound explanations that perpetuate subordination and oppression of Asian women. Findings from this study could also be applied to other young Asian women in similar demographic contexts.

\subsection{Implications for Nursing Practice}

To ensure safe and ethical care, nurses and health care professionals must interrogate discourses of power relations and social inequities, within micro- and macro- contexts, that shape the mental health vulnerabilities for young Asian women. Nurses must engage in ongoing critical reflection to recognize their own positions of power, assumptions about Asian and other 
racialized individuals and communities, and limitations in their own understandings about culture in order to avoid perpetuation of racialized-sexism, microaggressions, and internalized oppression in their practice.

On a broader level, our study supports the need for mental health initiatives that consider and address racism, discrimination, marginalization, and power relations that construct the everyday growing up experiences of young Asian women and other racialized youth.

Conventional models of "culturally competent care" frameworks fail to interrogate how power relations perpetuate the Othering of racialized communities and oversimplify the notion of culture (McGibbon \& Etowa, 2009). Mental health promotion interventions must go beyond "cultural" determinants and address the social contexts and everyday experiences of discrimination that shape the mental health vulnerabilities of young Asian women in Canada (Hilario, Oliffe, Wong, Browne, \& Johnson, 2018). Creating access and opportunities for meaningful engagement and dialogue wherein young Asian women (and other racialized youth) may share experiences and support one another is critical to promote mental health. Youthdriven interventions (i.e. peer counselling), that recognize the heterogeneity of their growing-up experiences and draw on their resilience, can foster individual and collective empowerment and action to support overall health and wellness.

\subsection{Conclusion}

This study contributes to the limited literature on the mental health vulnerabilities of 1.5 and 2.0 generation young Asian women by exploring their nuanced growing up experiences within both the mainstream "Canadian" and ethnocultural contexts. Applying intersectionality, CRT, and CFT revealed that contrary to current discourse, young Asian women's mental health vulnerabilities are not merely shaped by "cultural" determinants. Our findings reveal the 
complex interactions between Whiteness, power relations, and systemic inequality, and how they construct racialized-gendered contexts that shape young Asian women's mental health experiences. Although these racialized-gendered contexts produced constraints that increased their vulnerability to harmful mental health outcomes, it is critical to recognize that these women have their own strengths and strategies of resistance to cope, protect, and promote their own mental well-being. 


\section{Chapter 5: "Not Canadian Enough": Young Asian Women's Identity Construction and Mental Well-being}

\subsection{Introduction}

Navigating multiple sociocultural worlds can be challenging for adolescents, especially if they belong to ethno-racial minority communities. Although individual experiences vary across individuals, most racialized adolescents face nuanced contexts whereby the construction of their identities is straddled across broader constructs of culture, race, gender, and power relations. Research suggests that 1.5 generation and 2.0 generation individuals experience greater mental health challenges compared to their 1.0 generation counterparts (Islam et al., 2014; SuarezOrozco \& Qin, 2006). Specifically, their mental health status and resilience is associated with their experiences of having to simultaneously navigate and negotiate the norms and expectations of their "heritage" and "mainstream" Canadian sociocultural demands (Lee et al., 2009). These experiences have significant implications to their identity constructions and mental health outcomes (Vaghela \& Ueno, 2017) and therefore, merit a deeper and meaningful exploration.

Asian individuals are the fastest growing population in Canada (Statistics Canada, 2018b), and over half of them are women living in large metropolitan cities such as Toronto, Ontario (Chui \& Maheux, 2011). To explore how 1.5 and 2.0 generation young Asian women navigate their heritage and mainstream environments, researchers often rely on Berry's bidimensional acculturation theory. Acculturation refers to the changes that occur following continuous contact between individuals and groups from different cultural backgrounds (Sam \& Berry, 2006). However, dominant research on the acculturation experiences of Asian people in diaspora often negate the role of structural influences, such as power relationships and systemic inequity, in mediating ones" "acculturation" processes and outcomes (Chirkov, 2009a). Furthermore, these 
studies seldom consider young Asian women's racialized and gendered social positions and related everyday encounters that shape their "acculturation" experiences, identity construction, and mental health outcomes. Without incorporating a critical perspective, research that applies acculturation theories in simplistic ways can potentially pathologize young Asian women, perpetuate stereotypes (Ngo, 2008), promote victim-blaming, and limit mental health care to individual-level interventions (Viruell-Fuentes, 2007).

Although 1.5 and 2.0 generation young Asian women in Canada are projected to grow steadily (Statistics Canada, 2018b), there is a lack of research on their mental health experiences. Moreover, the paucity of critically-grounded research on their acculturation, enculturation, and mental health experiences is concerning. Most acculturation and enculturation studies ignore how unequal power relations across "dominant" and "subordinated" social contexts shape individuals' ability to negotiate, reconcile, and (re)construct their identities and mental wellbeing. There is a pressing need for research that critically examines acculturation within the context of asymmetric power relationships to inform effective and inclusive mental health programs and strategies to promote mental health equity. This study contributes to addressing the current research gaps by exploring the ways 1.5 and 2.0 generation young Asian women navigate their "acculturation" and "enculturation" experiences that shape their identity construction and mental health vulnerabilities.

\subsection{Literature Review}

\subsubsection{Dominant discourses on "Canadian" and "Asian" cultures}

Culture is a construct comprised of salient social systems (visible artifacts, behaviours, beliefs, values) through which individuals and communities organize and interpret their social worlds (Rudmin, 2009; McGibbon \& Etowa, 2009). Multi-dimensional in nature, culture can 
shift, interact, and change when people interact with one another (McGibbon \& Etowa, 2009).

Thus, culture informs individuals' social practice, and is known to have significant influence on their identities and health outcomes, especially for racialized groups (Levesque \& Li, 2014; McGibbon \& Etowa, 2009). It is important to note that the term "culture" is often used synonymously with the concepts of "race" and "ethnicity", leading to an oversimplified and monolithic understanding of "culture" as group traits and practices (Kumas-Tan et al., 2007). This understanding reinforces the discourses of "natural" traits and "traditional" cultures to reinforce stereotypes, negating the role of broader institutional systems in shaping such discourses (Duffy, 2001). Therefore, it is critical to interrogate current conceptualizations of "Canadian" and "Asian" cultures that provide the contexts wherein young 1.5 and 2.0 Asian women negotiate and navigate their identities and mental health.

Since changes to the long-standing exclusionary Canadian immigration policies were introduced in 1960s, Canada has admitted more racialized immigrants. The increased diverse population has been used to symbolize a "multicultural" society (Grant, 2016) and is boasted internationally as a key characteristic of "Canadian" society, reinforcing the discourse that Canada is a safe, welcoming, pluralistic, fair and democratic country (Lee \& Hebert, 2006). Little attention has been paid to the socioeconomic and political realities faced by Indigenous peoples and racialized groups, including income inequality, food insecurity, and access to meaningful employment and education (McGibbon \& Etowa, 2009). Simultaneously, the dominant Canadian culture has been constructed based on Western worldviews and colonial practices that construct personal traits, such as being individualistic, adventurous, daring, outspoken (Stroink \& Lalonde, 2009), sexually progressive (Suarez-Orozco \& Qin, 2006) and liberal (Li, 2009), as desirable and superior. Hockey, a cultural symbol central in the Canadian 
narrative, has been used to promote nationalism and a sense of communal belonging among citizens (Watson, 2017), which is not accessible to all Canadians. Outdoor wilderness activities such as snowboarding, skating, camping, and family cottaging are also promoted as key features of Canadian culture, and their symbolic significance continues to be passed on to future generations (Stevens, 2013). The ascription of these traits, beliefs, and practices as distinctly "Canadian" glosses over the critical issues of colonialism, Western dominance and White privilege, and perpetuates social exclusion of the Other, who do not have access to these cultural and symbolic capital, or are not able to find meaning in these symbols. As more 1.5 and 2.0 generation individuals self-report "Canadian" as their ethnic identity (Lee \& Edmonston, 2010), it is critical to interrogate the concept of culture through the lenses of colonization, Whiteness, and unequal power relations.

While young Asian women are exposed to diverse cultures in Canada, their growing up experiences and identity construction are primarily connected to their immediate environment, shaped by their interactions with their parents, families and relatives $(\mathrm{Li}, 2009)$ but also in the context of the ever present dominant White Canadian culture. Western scholars often view "Asian" parenting as authoritative and over-involved, characterized by high parental pressures for their children to perform "culturally" desirable behaviours (Choi et al., 2017) such as: achieving academic excellence (Tummala-Narra et al., 2016; Yoon et al., 2017), maintaining emotional restraint (Lipsicas \& Makinen, 2010), familial and filial subservience (Vu \& Rook, 2012), social conformation to preserve harmony (Shea \& Yeh, 2008), and retention of Asian heritage (Rhee et al., 2003). Furthermore, the worldwide practice of patriarchy is often negated in discussions about "Asian" cultures. As such, Asian communities are portrayed to be gender restricting and patriarchal, (re)producing the stereotypes that Asian women are expected to 
maintain cultural continuity, chastity, subservience, and domestic practices (Chang et al., 2017), while males are encouraged to pursue education and career aspirations (Vu \& Rook, 2012). These discourses negate that patriarchy exists in all cultures and societies and are not natural attributes of any particular culture.

Existing literature also suggests that young Asian women's mental health vulnerabilities are frequently attributed to influences of "cultural" and "gendered" norms that emphasize emotional restraint to avoid being burdensome (Alamilla et al., 2017) and drawing negative attention to their family (Shea \& Yeh, 2008). Within this literature, intra-group variability across Asian cultures is often minimized (Fung \& Wong, 2007) and racist stereotypes about Asian individuals and communities are perpetuated. Relatively few studies recognize how the so-called "Asian" cultural traits are implicated within systematic and institutionalized power relations based on racism, sexism, and classism. Without this perspective, we continue to benchmark young Asian women's experiences against dominant White "Canadian" cultures to reinforce an oppressive practice that reproduces social inequality and mental health vulnerabilities.

\subsubsection{Current Conceptualization of Acculturation and Enculturation}

Since Redfield, Linton, and Herskovits (1936) first defined acculturation as "those phenomena which result when groups of individuals from different cultures come into continuous first-hand contact, with subsequent changes in the original cultural patterns of either or both groups" (p. 149), acculturation theories have evolved to capture bidimensional changes that occur between the mainstream (defined as dominant) and the heritage (defined as ethnic) society (Sam \& Berry, 2006). Although variations in the definition and operationalization of acculturation remain elusive and inconsistent across the literature, many researchers consider the concept a key variable in the mental health practices and outcomes of racialized individuals (Nap 
et al., 2014; Yoon et al., 2013). Of these conceptualizations, John Berry’s bidimensional acculturation theory (1980) is the most cited and prominent (Zhang \& Moradi, 2012). Berry suggests that it is plausible for an individual to be simultaneously oriented to their mainstream and heritage culture, and changes may occur along two independent dimensions (Berry, 1980). As such, there are four possible acculturation outcomes: integration (maintaining both mainstream and heritage culture), assimilation (maintaining the mainstream culture while rejecting/avoiding heritage culture), separation (maintaining heritage culture while rejecting/avoiding mainstream culture), and marginalization (rejecting/avoiding both heritage and mainstream culture) (Berry, 1980). While this dominant theory offers multiple outcomes, theorists primarily focus on how non-European immigrants "acculturate" towards the mainstream Westernized culture in a unilinear manner (Viruell-Fuentes et al., 2012; Ngo, 2008). Some researchers argue that "acculturation" may not comprehensively capture the experiences of Asian individuals born into the mainstream culture (i.e. 2.0 generation), some of whom are not fully oriented into their Asian heritage (Kim et al., 2009). Therefore, the concept of "enculturation" was used to refer to the process of learning or retaining the norms, values, and behaviours of the heritage culture (Kim et al., 2009). Enculturation promotes an equal level of emphasis on learning and retaining heritage norms as much as "acculturating" (Kim, 2007) and is a critical concept in research of identity construction.

Despite evidence that experiences of acculturation and enculturation shape mental health (Yoon et al., 2013), identity development (Hilario et al., 2014), and sense of self for racialized and ethnic minority individuals (Cheng et al., 2014), research in these two areas remain limited and results are inconsistent for Asian populations. For instance, some studies report that Asians in diaspora who are more "acculturated" report lower levels of psychological distress (Ruzek et 
al., 2011), anxiety (Farver et al., 2002), acculturative stress (Miller et al., 2011), and higher levels of positive self-esteem (Farver, et al., 2002), better mental health outcomes (Alamilla et al., 2017), and use of mental health services (Shea \& Yeh, 2008). Conversely, other studies suggest that higher levels of acculturation may lead to deleterious outcomes such as increased stress (Choi et al., 2017), family conflict (Choi et al., 2016), and perceived discrimination (Tummala-Narra et al., 2016). One explanation for the positive outcomes could be that by adopting and maintaining the norms and values of the mainstream culture, 1.5 and 2.0 generation Asian individuals are more likely to feel a sense of belonging (Choi et al., 2017) and better able to avoid feelings of exclusion from their mainstream peers (Tummala-Narra et al., 2016). However, this same practice may render them vulnerable to Othering, as their racialized differences are visible in the mainstream contexts and considered "not" Canadian, and their behaviours are perceived as misaligned with their Asian heritage (Choi et al., 2017; Ruzek et al., 2011). These findings suggest a need for further exploration of the acculturation experiences of 1.5 and 2.0 generation Asians in diaspora.

On the other hand, existing research posit that enculturation is beneficial to the mental health of Asian youth by promoting positive self-concept (Kim \& Omizo, 2010), sense of cultural identity and belonging (Alamila et al., 2017), familial harmony (Choi et al., 2016), access to heritage and familial resources (Tummala-Narra et al., 2016), and buffering against stress and discrimination (Alamilla et al., 2017; Tummala-Narra et al., 2016). By maintaining their Asian heritage, they may feel more supported in their ethno-cultural communities and more confident in their ability to navigate the demands of the mainstream culture (Kim \& Omizo, 2010). 
Enculturation may also foster appreciation and identification with their Asian heritage; promoting ethnic pride, and the perception of a stronger bond with their parents and co-ethnic peers (Choi et al., 2016; Kim \& Omizo, 2010). These findings suggest that young Asian individuals' enculturation experiences are not as deleterious to their mental health and identity development. This encourages a deeper exploration of acculturation scholarship that potentially problematizes individual's association and identification with their heritage culture.

Furthermore, acculturation and enculturation studies were primarily quantitative in nature, which utilize standardized scales to measure "acculturation outcomes" without accounting for the socio-political and historical contexts. These studies use "hypothetico-deductive" approaches to discover probabilistic associations between individuals' "acculturation" and mental health outcomes (Chirkov, 2009b, p. 178). Without contextualizing acculturation and enculturation experiences under broader societal discourses, we perpetuate the assumption that racialized individuals possess the "free will" to determine their outcomes in the mainstream society, and undermine the Othering processes that produce experiences of discrimination and social exclusion (Ngo, 2008) and shape identity construction.

\subsubsection{Identity Construction and Mental Health of Young 1.5 and 2.0 Asian Women}

The intersection of racism and sexism produce nuanced contexts that shape the mental health and identities of young Asian women as they experience racialized-sexism (or genderedracism; Essed, 1990). Racist and sexist stereotypes that portray them as exotic, inevitably smart, foreign, submissive, or passive perpetuate mental health vulnerabilities and feelings of exclusion, anger, and stress (Mukkamala \& Suyemoto, 2018; Sue et al., 2007). Furthermore, there is evidence to suggest a positive association between generational status (in the context of migration and nativity) and poor mental health outcomes for Asians in diaspora (Alamilla et al., 
2017). Specifically, 1.5 and 2.0 generation Asian individuals experience greater mental health challenges compared to their 1.0 generation counterparts (Islam et al., 2014; Lee et al., 2009). These individuals immigrate at a young age (1.5 generation) or are born in the mainstream country (2.0 generation) and construct their identities in unique sociocultural contexts, whereby they negotiate between contrasting yet parallel influences from their heritage and the mainstream cultures (Islam et al., 2014; Wu et al., 2012). For instance, although most 1.5 and 2.0 generation children are expected to have similar values and practices as their parents, they face external pressures to fit in with their mainstream peers. Adolescence is a period of time when young people assert independence, learn different social roles and rules, and experience peer pressure and competition for popularity (Li, 2009). Crossing cultural boundaries may render them vulnerable to mental health stresses and identity tensions (Tummala-Narra et al., 2016; Ying \& Han, 2007) as they adopt certain behaviours in order to "belong" and fulfill perceived expectations from both cultures.

Some authors argue that gender matters as Asian women are pressured to bear additional responsibility of maintaining their heritage culture more than their male counterparts (Leu et al., 2011). Additionally, many 1.5 and 2.0 generation racialized women grow up in Othering environments, where they learn about their difference within the mainstream culture (Rajiva, 2006). These spaces of White dominance and patriarchy impose and reinforce their "inferior" status as racialized women at a young age, perpetuating mental health stresses associated with chronic experiences of oppression, prejudice, and racial discrimination (Sue et al., 2007). Given that experiences of acculturation and enculturation produce long-term impacts on identity development (Goodenow \& Espin, 1993) and mental health for Asian youth (Hwang \& Ting, 2008; Miller et al., 2011), there is a critical need to explore the growing up experiences 
of 1.5 and 2.0 generation Asian women in the context of power relations manifested as racialized-sexism. To this end, this manuscript-style chapter explores the following research questions: 1) How do the dominant discourses of "Canadian identity" and "culture" shape the intertwined social identities and growing up experiences of young 1.5 and 2.0 generation young Asian women? and 2) How do young 1.5 and 2.0 generation Asian women's growing up (acculturation/enculturation) experiences affect their mental health?

\subsection{Methodology}

This narrative research drew on CFT and CRT to explore the growing up experiences of young Asian women within the broader concepts of culture, enculturation, and acculturation. Narrative research centralizes individual subjectivity; how we understand and organize our past and present experiences that are shaped primarily by micro-, meso-, and macro discourses (Reissman, 1993). Within an integrated lens of CFT and CRT, a narrative approach offers an insight into how young Asian women interpret their interactions within their heritage and mainstream contexts largely shaped by power relations, social inequity, and racialized-sexism (Holloway \& Freshwater, 2007b). While young Asian women's narratives are not the absolute representation of their experiences, they facilitate a deeper understanding of their pathways to identity construction and how these processes affect their mental health. In addition, concepts of acculturation and enculturation support our critical analysis of the often taken-for-granted notion of "culture". However, we have gone beyond applying these concepts to describe how individuals in diasporic communities adopt mainstream cultural practices or maintain their heritage cultural practices, to situate acculturation and enculturation within the broader contexts of power relations based on racism, sexism, and classism. 


\subsubsection{Recruitment}

Young women living in the Greater Toronto Area in Canada were invited to participate in our study. Ethics approval was obtained from Ryerson University. Participation inclusion criteria were: aged 18 to 23, self-identified as a woman from East, Southeast, or South Asian background, born in Canada or have lived in Canada for at least seven years or more, living in the Greater Toronto Area. Purposive sampling was used to recruit participants. Recruitment strategies included in-person outreach, posting of flyers at community organizations and student associations, and distributing e-flyers to existing networks serving youth. Flyers described the topic of inquiry (young Asian women's growing up experiences) and encouraged participants interested in sharing their experiences to contact the lead author through email or phone.

\subsubsection{Participants}

The study sample consisted of 14 self-identified young Asian women between the ages of 18 to 23 . Of all participants, nine were born in Canada to at least one first generation parent. The study participants self-identified with seven Asian ethnic backgrounds. Thirteen participants completed elementary education in Canada. During the time of the study, participants were attending high school $(n=1)$, college or university $(n=10)$, working in professional settings $(n=2)$, or graduated and applying to law school $(\mathrm{n}=1)$.

\subsubsection{Data collection}

Data collection involved focus group and individual interviews. Participants were divided into three groups: Group $1(\mathrm{n}=4)$, Group $2(\mathrm{n}=6)$, and Group $3(\mathrm{n}=4)$, and attended three semistructured focus groups at least one week apart. Each focus group interview was facilitated by the first two authors and two research assistants, and lasted approximately 2.5 hours each in a private meeting room at Ryerson University. Twelve of the 14 participants took part in all three 
focus groups. Two participants did not return after the first focus group due to scheduling conflicts. Individual interviews were held with a subset of nine consenting participants, which allowed for a richer and deeper exploration of participants' acculturation and enculturation narratives.

Informed written consent were obtained from all participants prior to all interviews. The focus group and individual interviews were semi-structured and dialogical in nature, engaging participants in critical reflection of: 1) their growing up experiences at home and at school, 2) how racialized and gendered experiences informed their identity construction, and 3) their voices

of resilience and vision for an equitable world for young Asian women. Participants were given a signed copy of their informed consent forms, list of relevant community and health organizations and resources, an honorarium of $\$ 20 \mathrm{CAD}$ and two public transit fares in appreciation of their time and contributions. All interviews were audio-recorded and transcribed verbatim by the research team.

\subsubsection{Data analysis}

We used an interpretive thematic approach to analyze the data. Drawing on Critical Narrative Analysis (CNA), we explored how individuals' identities and narratives are shaped by their lived experiences, which are inseparable from the social institutions and systems, and how systematic discourses are, in turn, shaped by individual narratives (Souto-Manning, 2014). Themes were inductively (shared by participants) and deductively (guided by theoretical framework) identified through repeated listening of the interviews and reading of de-identified transcripts to gain familiarity and a broad understanding of the participants' narratives (Patton, 2002). A codebook of key themes and subthemes with definitions was developed based on team discussion. N-Vivo 11 was used to aid line-by-line coding of key themes that were specific to: 
the research questions, concepts and ideas articulated by the participants, and theoretical concepts relevant to this study. Coding was reviewed and agreed upon by at least two team members. The first author also produced a 2-page comprehensive summary of each transcript to ensure that the contexts and nuances of the focus groups were not lost through the process of disaggregation and coding of data (Wong, 2014). All authors collaborated in reviewing the analyzed data and application of theoretical frameworks in data interpretations. Participants' names were replaced with pseudonyms to protect their identities.

\subsection{Results}

Analysis of the participants' narratives suggested that their understanding of and responses to the current discourses of "Asian" and "Canadian" cultures were primarily shaped by dominant stereotypes and perceived values of their heritage and mainstream culture. These perceptions (along with racialized-sexism) influenced the way they negotiated their situational identities in response to the complex social contexts they found themselves in. For most participants, their experiences of constructing and negotiating situational identities often produced tensions, conflicts, and an avoidance to stress, which resulted in mental health responses that varied from disempowerment to self-pride.

\subsubsection{Being "Asian" in the "Canadian" Context: Whiteness as benchmarking}

To gain a deeper understanding of young Asian women's “acculturation" experiences, it is critical to interrogate their cross-cultural contexts, primarily defined by their perception of what constitutes "Asian" in relation to the "Canadian" culture. Participants often drew parallels by benchmarking it to discourses of what it meant to be "Canadian". Being "Asian" was defined in contrast to the seemingly more 'liberal' norms of the "Canadian" culture, which some found to be more appealing compared to their 'rigid' heritage culture: 
"I feel like Western culture is so much more forgiving because Asian culture... it has all these expectations... you're supposed to know how to cook the food, how to treat your elders, table manners, etc. Western culture is so much more broad... I feel more comfortable there because I don't have to fit into all these rules," (Min-Jung, 21, Korean, 1.5 generation).

Participants also defined the "Asian" culture by what it was not; portraying a "grass is greener when you're Canadian" narrative: "Western culture is more individualistic than traditional Asian culture...you're allowed to create your own identity... it's more progressive in a lot of ways," (Yun-Hee, 22, Korean, 2.0 generation).

Participants described the "traditional" Asian culture in a manner that reinforced current stereotypes such as emphasis on academic success, financial stability, familial obligation, and emotional restraint. Kelly considered herself more "Canadian" than Asian by claiming that such stereotypes were not applicable to her: "I think I integrated pretty well into the Canadian culture. I identify really well with the North American lifestyle... I think a lot of the stereotypical Asian family dynamics...is not really relevant in my family," (22, Chinese, 2.0 generation).

Some shared growing-up expectations they perceived to be associated with both their racialized and gendered positioning: behaving politely, pursuing long-term romantic relationships, and being “conservative” as emphasized by Kameela here: “you can’t be too expressive of who you are... you have to wear a covering, clothes, not anything flaunting, anything that can attract the wrong kind of attention... all the time growing up, I'd wear conservative clothes," (19, Pakistani, 1.5 generation). 


\subsubsection{Being "Fresh-off-the-boat" or "Whitewashed" Asians}

In a similar vein, being "Canadian" is characterized by someone who "goes against the stereotypical Asian: being very docile, not speaking loud, not speaking back to anybody," (MinJung, 21, Korean, 1.5 generation). By pursuing a career in fashion, Meniza described herself as the "black sheep" in her Filipino family:

"I feel like I don't fit in really like when I hang out with my family... I'm already in fashion, like the most un-Asian thing to go into. It's like pretty different and I guess, just not sticking to conventions and being more out there," (Meniza, 19, Filipino, 1.5 generation)

Some participants attributed being "Canadian" to participating in activities (such as sleepovers, cottages, camping, snow sports) that they did not experience previously by virtue of growing-up in their "Asian" families.

Interestingly, some participants recognized the diversity among Asian people based on their perceived "acculturative" locations. For instance, a distinct differentiation was made between "FOBs" (fresh-off-the-boat) and or "whitewashed" Asians. The latter term was used to describe those who "acculturated" by adapting the perceived norms and behaviours of the mainstream culture such as, "speaking up in class and say what they want to say... they were advanced, even in the sexual world... they would hang out after school together, go to their boyfriend or girlfriend's houses" (Min-Jung, 21, Korean, 1.5).

The term "FOBs" was also used by participants to socially categorize their co-ethnic peers based on their own social locations. Specifically, "FOB" referred to those who embodied certain ethnic identifiers (as defined by the participants and the dominant discourse): speaking English with an accent, speaking their heritage language with other co-ethnic peers, eating ethnic 
food in social spaces, or engaging in behaviours associated with those who retain their heritage cultural practices (such as listening to Asian music or frequenting local Asian enclaves).

These discourses influenced the extent to which participants identified with a particular nationality. Participants who self-identified as "Canadian" regarded being "whitewashed" as a more desirable status. While they did not explicitly reject or ridicule "FOBs", they constantly reiterated their perceived distance from their heritage culture, and thus, alluded to a direct juxtaposition from "FOBs". Yun-Hee explains: "I didn't associate with (FOBs) at the time because I was so uncomfortable with being Asian. So because I didn't want to eat in the cafeteria and be part of the Asian crowd, the Korean friends that I do have are the whitewashed Korean," (22, Korean, 1.5 generation). When asked why she calls herself "whitewashed", Faye shared her discomfort in identifying with her heritage culture:

“I can't do anything Asian. I can't speak Chinese, so that kind of cuts me off right away...I think my mom said this once, that Asians kind of expect the girls to be a lot more introverted, polite, and...I'm none of those things. So I just didn't fit in well there... I am just nothing like that," (21, Chinese, 2.0 generation).

Faye's narrative reiterates the aforementioned benchmarking practices through which some young Asian women interpret their "whitewashed" identities as juxtapositions to their "Asian" heritage. In addition, it also highlights the centrality of external behaviours in characterizing an individual's "culture" (i.e., speaking a foreign language, fashion, listening to 'ethnic' music), which limits its' boundaries and negates other facets. 


\subsection{3 "Not that Asian": Negotiating "Acculturation" and Identities}

Participants' narratives suggest that their identity construction and acculturation experiences were conditioned by multiple social processes, whereby they simultaneously reconstructed and deconstructed their "selves" in response to their perceived demands from their heritage and mainstream contexts. When asked about their growing up experiences in the mainstream "Canadian" context, some participants shared their exposure to being "Othered" at a young age, and these experiences pushed them towards adopting certain behaviours to fit in with their mainstream peers:

"When I brought my lunchbox to school and people made fun of what I was eating, it made me more Westernized... I really was not proud of my identity or culture at that time...I really focused a lot on trying to fit in by looking at mainstream music, mainstream pop culture, like Western things," (Yun-Hee, 22, Korean, 1.5 generation).

This pervasive Othering of their "Asian" identity made some participants feel ashamed and rejected their heritage culture in pursuit of their "Canadian" identities. In social contexts wherein young Asian women are visibly different from their mainstream peers, participants shared their experiences of racial microaggressions ${ }^{15}$ :

"Everyone in school assumed I was good at math and all asked me for like my calculator... There was this one dude who was Chinese who was in my class, he was better at math than the teacher... Because of that, other people started assuming I would be good at that," (Faye, 21, Chinese, 2.0 generation).

\footnotetext{
${ }^{15}$ Racial microaggressions are brief and everyday exchanges that communicate derogatory racial insults towards people of colour (Sue, 2010). Whether intended or not, they are often "subtle" insults that are dismissed as innocent or innocuous with detrimental physical, emotional, and mental effects (Sue, 2010).
} 
In this and other narratives, the young women shared experiences of "cultural imposition ${ }^{16 "}$ " that heightened their awareness of being the Asian "Other". Despite self-identifying as being more "Canadian" than "Asian", most participants encountered exchanges that reinforced their tacit knowledge that they were racially different, as Yun-Hee shared: "If a friend introduces me to another friend, they'll say, 'she's Korean but not that Korean,' they mean it as a compliment. 'Not that Korean' in that I don't have an accent, so I guess that's my power," (22, Korean, 1.5 generation). Faye described another experience of cultural imposition:

"In the end people have always been like, 'Oh you're Chinese, you know, just don't forget that you're Chinese.' Or, 'Oh you're Asian, what part of Asia are you from?' So I'm always constantly reminded that I'm Asian...I'm definitely much more Canadian than I am Asian," (21, Chinese, 2.0 generation).

Other participants' narratives also illustrated that racist stereotypes were perceived as negative and degrading, which led those who considered themselves more "Canadian" to avoid exhibiting behaviours that may identify them as "Asian". Amita explained why she refused to get out of the car to go into a store after attending a wedding and wearing Indian clothes:

“I didn't want to attract attention because it's different. I still feel the same way today...I don't feel comfortable in cultural clothing because it's different. I guess it's because of the negative stereotypes associated with being Indian, like they all smell or they all like curry...And I know I'm still kind of visible, but if I don't wear cultural clothing, it will be less predominant," (Amita, 23, Indian, 2.0 generation).

16 "Cultural imposition" refers to the practice of imposing beliefs, values, and assumptions upon another individual (Leininger, 1991). 
In this account, Amita expressed awareness of her identity as an Indian woman and her perception that minimizing her visibility as an ethnic minority is a prerequisite for social inclusion in the "Canadian" mainstream.

On the contrary, some participants had turned their encounters with racial prejudice into inspiration to connect with their Asian heritage to challenge dominant stereotypes. Kameela wore a hijab as her strategy of resistance and to dispel stereotypes:

“I wanted to show people I'm Muslim, I'm Pakistani... you cannot associate me with a stereotype and a general idea of what you think a culture is because in every culture and every country there are certain people... that do certain things that unfortunately end up identifying that community... and I wanted to show people that, that's not who I am, that's not what most of the people in my country or community are... I wanted to be an ambassador," (19, Pakistani, 1.5 generation).

\subsubsection{Creating In-between Cultures and Identities}

Some participants shared that growing up in primarily White social spaces influenced their personal preference of "Canadian" music, fashion, and movies, thus helping them "blend in" with their mainstream White peers. Despite this, however, some participants recognized the limits of their ability to truly "blend in" with the mainstream "Canadian" culture. Min-Jung, who considered herself "whitewashed", shared an experience that questioned her sense of belonging:

"I've been dating my boyfriend...He's white... We were at a bar with a live band playing all this really white music that I didn't know and people were like, 'how do you not know this person?'... Everyone was singing along and I was just like, 
I don't know this. I can't learn this type of stuff. I don't know it the way they do," (21, Korean, 2.0 generation)

Some participants perceived that their ability to associate with the mainstream "Canadian" culture was limited by their heritage "culture" that enforced gendered expectations:

"Because of the way my culture is... my father is more protective of us. So hanging out with friends and participating in extra-curricular activities wasn't something that came easy to me... Everything was a lot more strict. For my family, that sense of safety only came from people who were of my (ethnic) background... I guess I've been really introverted opposed to extroverted because I've been really confined. (Fathima, 22, Sri Lankan, 1.5 generation).

For others, their "Canadian" identity construction was influenced simultaneously by the negative connotations associated with their "Asian" heritage, as well as lack of ethnic representation and celebration in the mainstream culture:

"In elementary school, I got the idea that to be Indian was a minority...I got the sense that those were negative... You don't get to see the South Asian culture celebrated. Until I saw Bollywood when I was in grade 3 or 6, I didn't get to see it, so I didn't have that positive reinforcement," (Sabrina, 21, Indian, 1.5 generation).

Sabrina's narrative illustrated how dominant discourse and media shaped perceptions about their heritage culture, while watching Bollywood also provided access to connect with her Indian culture. However, for Amita, this experience fostered a sense of misalignment and heightened an awareness of her positioning "in-between" her heritage and mainstream spaces:

"I started watching Indian films... just noting how it's kind of different from 
like, the Canadian culture...so like the next day when I go to school, it's like a different culture... The culture that you have at home is different...from other people's culture...you notice that difference," (23, Indian, 2.0 generation)

Despite identifying as a "Canadian", Meniza's narrative refers to this "in-between" positioning and illustrates how socio-environmental contexts foster different situational identities. She spoke about her interaction with her mainstream "Canadian" peers:

"You kind of learn to walk like them, be like them...because you hang out so much with them. By the end of the day... you come back to your home, your family, and even though you learn to do this, you kind of have to revert back to your mannerisms at home" (19, Filipino, 1.5 generation).

Many participants connected with their "Asian" culture through language, music, and food as primarily taught by their parents or grandparents. However, for Kelly, finding comfort in this "inbetween" space was challenged as she felt that her 1.5 generation parents could have tried "harder" to teach her about her Chinese culture. She describes this conflict here: "I absolutely love the Asian culture... I'm kind of upset my parents didn't try a little harder to do that...They didn't send me to Chinese school," (22, Chinese, 2.0 generation). On the other hand, some participants also shared a sense of cultural pride and belonging that was fostered by their families' unconditional love and acceptance:

"I was taught just to be myself. I'm just proud of being Asian... I feel like I fit in with my family. I can just say whatever I want and they'll accept me. If I marry a white man, they'll still love me and be happy for me (Hanh-Ly, 18, Vietnamese, 2.0 generation). 
In this narrative, Hanh-Ly did not associate being "Asian" with "traditional" ethnic identifiers of language, music, or food. Rather, her cultural pride and identity stemmed from her family's acceptance and support.

Some participants internalized the perceived values of their "Asian" heritage culture like academic success. For instance, some young women shared on how their academic expectations were shaped by their heritage communities: "There are the respected professions: lawyers, nurses, doctors... I've really internalized that ideology. I've studied environmental studies and now I'm going to take my LSATs. It's hard to say if I am really interested in law or if I want to get respect from my community" (Yun-Hee, 22, Korean, 1.5 generation). This in-between identity was crafted to balance fusion of the perceived desires of her heritage community with her own personal interests.

Others reflected on how navigating their academic paths was influenced by their parents' post-migration challenges, to some extent. Rubylyn recounts: "For us here, we have everything right at our finger tips. It's just a matter of holding onto it, or grabbing it or reaching for it. So I

think like for my dad... it's more like, 'security, security.' You need something so you can live," (21, Filipino, 2.0 generation). While she acknowledged her parents' perspective about financial stability, Rubylyn continued to pursue her own educational aspirations based on her own interests. This experience led her to an in-between place: she shared that her decision to go into event planning had initially led to familial conflicts but that was resolved with improved communication with and increased support from her parents.

\subsubsection{Constructing Multiple Situational "Selves" and Mental Well-being}

Participants' narratives suggest that their identity construction and mental well-being are largely shaped by power relations within and across their heritage and mainstream contexts. 
Constructing and performing in-between identities often required the parallel construction of multiple situational "selves", which involves exhibiting certain attributes and/or performing certain identities to achieve a certain outcome. Most participants spoke about the strategies they had used to navigate and reconcile their personal interests, the norms and demands of their heritage and mainstream Canadian cultures, and everything in between. When it came to developing a situational self, Amita explained:

"It depends on who you're with, but also depends on the environment that you're in. If you're with people who are in your culture, like we are now, we consider ourselves to be more Asian. But if we were to go back to our friends from other cultures, we would adapt to that scenario/environment and interact in a more Westernly manner," (23, Indian, 2.0 generation).

Amita's narrative illustrated the complex ways young Asian women negotiate and express their identity. For some participants who considered themselves as "Canadian", their "Asian" selves were something to compartmentalize and accessed only within their heritage settings: "I package like my Asian cultural identity thing into a neat little package and put it on the side for now," (Min-Jung, 21, Korean, 2.0 generation). This strategy has led to identity tension and psychological conflicts when these young Asian women had to lead a double-life that required them to put either their "Canadian" self or their "Asian" self in closets, as Meniza described: "I stick out so much as a Canadian...my parents raised me with the values of like modesty and female roles and all that stuff...I'm like no, I don't want to get married, I don't want to have kids, like I don't care... I'm much more outspoken than that. I have to repress that...like there's like a whole other side (to me)," (19, Filipino, 1.5 generation). 
Balancing multiple situational identities created confusion surrounding their "true" selves. On the occasion in which participants would perform being "Canadian" to fit in with their mainstream peers, they also experience inner conflicts and tension. They felt a sense of belonging with the "in-group", while simultaneously feeling guilty and stressed for engaging in certain behaviours that their parents may perceive as 'undesirable'. Many participants spoke about the tremendous mental energy required to navigate complex situations and determine which identity can help them meet their desired outcome:

"There is a way that I would act at home, which is kind of different from the way I would at school, which is different from the way I would act at work...I adapt to my environment depending on the expectations of me... then after all that happening in a day, I don't exactly know which one of them is me... the time that I am by myself, I am just totally exhausted by all the roles and acting that I've done the entire day," (Kameela, 19, Pakistani, 1.5 generation)

Despite the conscious effort and labour spent on trying to fit in and meet the expectations in multiple contexts, participants still experienced challenges that made certain "identities" inaccessible.

Repeated encounters of cultural imposition from their mainstream peers made some young Asian women feel Othered and disempowered. The expectation for them to be a "cultural ambassador" not only made some participants feel uncomfortable, but also reminded them they were not considered "Canadian".

"The expectation of people is that you're an ambassador of your culture. And I'm so uncomfortable... I feel like I don't know a lot about Korean culture or history...It would bug me when I would go over to friend's houses for dinner 
and their parents would ask me to tell them about my culture... I don't know anything," (Yun-Hee, 22, Korean, 1.5 generation).

However, it was also possible to feel out of place within their own ethnic community. Dalila shared this experience while engaging with her university's Tamil Students Group:

"A lot of my friends all of a sudden were Tamil... But then I stuck out within, because I didn't listen to the music... I wasn't comfortable speaking Tamil with friends... For some reason I'd feel uncomfortable,” (23, Tamil, 2.0 generation). Dalila's narrative demonstrates that despite her efforts in connecting with her heritage culture, social contexts and conditions continue to affect individuals' sense of belonging.

For some participants, experiences of cultural imposition provided them an opportunity to advocate for their heritage community. When Dalila experienced prejudice from a bus driver in relation to the political climate of Sri Lanka, the country she migrated from, she turned this negative experience into resistance:

"People would make assumptions or assertions about your beliefs or what you represented based on something that they read in the news... and that's when I started to understand how other people saw me... I started to become more involved because I felt this pressure of like, 'This is my label, okay let me do something proactive with it.' So I started to raise awareness or try to talk about humanitarian issues," (23, Tamil, 2.0 generation).

Most participants shared how these encounters of Othering contributed to their mental health vulnerabilities and/or resilience. Some young women perceived these encounters as opportunities to reconceptualize their relationships to the mainstream and heritage worlds and reconstruct their identities in their own way: 
"I think I had the advantage of kind of being able to see like, from both spheres, which is kind of a good thing about being raised in the West but having a little bit of an idea of what it's like in the East. Because you kind of get to see like how both of them function... and you try to take the best of it - best of both worlds, to the best of my ability anyway," (Faye, 21, Chinese, 2.0 generation).

Locating herself in the "in-between" world enabled Faye to develop a deeper, more nuanced understanding about her social positioning and how to blend certain elements to construct her identity. For other participants, this reconciliation between cultures fostered a critical selfreflection on the complex, multi-dimensional, yet restricting nature of labels:

"I do identify as Tamil. Do I identify as Canadian? Yes, but like it's a weird dynamic for me... What makes someone Canadian? Is it like those stereotypical like, 'Oh I watch hockey, and I like maple syrup.' '... It becomes problematic... like, who's considered as is and isn't?... I do acknowledge this is where I was born, and this is my ethnic background, and this is my heritage and all of that, so I do identify with that. But I feel like, once we start boxing ourselves into this, like, am I supposed to represent this?... So there are certain times that we might want to identify with it for whatever reason. But there are other times that we just say, 'No, because I don't want you to judge me and label me,' (Dalila, 23, Tamil, 2.0 generation).

While Dalila's narrative highlighted some of the tension involved in selecting and performing certain identities, she also referred to the fluidity and flexibility of the whole process. She demonstrated agency and resistance despite the constraints imposed on her by broader social contexts. 


\subsection{Discussion}

The following section offers a critical examination of Berry's bidimensional acculturation theory in light of young Asian women's experiences of identity construction and mental wellbeing within their heritage and mainstream contexts. Berry's bidimensional approach assumes that adopting a new (mainstream) culture does not lead to losing one's heritage culture since the change occurs on two independent dimensions (Berry, 1980). Our analysis revealed the insufficiency and incomplete nature of this framework in explicating the ongoing negotiation, navigation, and (re)construction of participants' identities, which were contingent on the broader discourses of asymmetric power relations.

The bidimensional framework promotes a reductionistic approach to analyzing the participants' experiences. For instance, participants who adopted "Canadian” identities and behaviours (i.e. identifying as "whitewashed") and resisted their heritage culture, would be considered "assimilated" as per this bidimensional approach (Berry, 1980). However, in the mainstream contexts where being "Asian" is not perceived as desirable, participants experienced both external and internal pressure to take on and perform their "Canadian" identities.

Participants, who have internalized racism and White supremacy, tried to cope with prejudice and racist microaggressions by distancing themselves from their "Asian" cultures and their coethnic "FOBs". This adaptive response served as a self-preserving mechanism to maintain their sense of belonging and identification with their White "Canadian" peers and minimize their experiences of being Othered. However, in doing so, they were also implicated in the act of Othering towards their heritage communities and newcomers.

In the same vein, the bidimensional framework would place participants who maintained their heritage engagement and pride while resisting the mainstream culture into the category of 
"separation" (Berry, 1980). Although this alludes to a notion of agentic choice on the participant's behalf, this simplistic categorization downplays the role of broader socio-cultural and political structures that (re)produce sites of oppression for racialized groups and warrant such acts of "resistance". Our finding suggests that existing acculturation theories are inadequate in explicating acculturation as processes and outcomes that are largely determined through power relations and existing social hierarchies. The adoption of "Canadian" and rejection of "Asian" identities (and vice versa) are intertwined with internalized oppressions, thus facilitating a false sense of self that may be perceived as "assimilation" or "separation".

Participants" interactions with and interpretations of the dominant discourses on "Asian" culture influenced the way they (re)constructed their identities within different social contexts. Their understanding of "traditional" Asian cultures was primarily shaped by mainstream discourses that define "Asian-ness" in negative and derogatory terms (i.e., as perpetual foreigners, non-progressive, and inferior; Sue et al., 2007) and "Asian" femininity as uniformly docile and submissive (Mukkemala \& Suyemoto, 2018). In the absence of critical interrogation, these unchallenged biases can substantiate research findings that associate increased "acculturation" (or assimilation) to the mainstream culture with improved mental health outcomes (Alamilla et al., 2017). This homogenizing and oppressive discourse normalizes dominant Whiteness as the status quo and promotes mainstream identification as worthy goals to achieve (Ngo, 2008). This discourse reinforces White normalcy and superiority of the "Canadian" identity above others. Subsequently, a static and stereotypical definition of "Asian" essentializes and pathologizes this group; justifying acculturation-based explanations that ascribe poor mental health outcomes to the seemingly "restrictive" and "old-fashioned Asian culture" 
(Viruell-Fuentes, 2011; Rudmin, 2009), negating the inequitable resource distribution that compromise Asian women's mental health .

Whether unintentional or not, the bidimensional acculturation framework reinforces unchallenged assumptions on the "inferiority" and "problems" of racialized minority groups, while simultaneously normalizes Whiteness as the benchmark. This practice subordinates Asian women and communities to a system that benefits from gender and racial oppressions and conceal the socio-political and historical contexts that perpetuate these oppressions (Gee et al., 2009). To illustrate, some participants experienced racialized-sexism through perceived pressures from their mainstream and heritage contexts to conform with notions of "Asian femininity" (i.e. being domestic and sexually submissive). As such, "Asian” women are depicted as subordinated beings in comparison to White women, who are perceived to be monolithically assertive, independent, and self-confident (Pyke \& Johnson, 2003). This construction of hegemonic White femininity reinforces the myth of "Canadian" ideals that promote selfsufficiency, autonomy and gender equality (Wong \& Tsang, 2004). Participants who internalized this discourse considered race as the basis of their understanding of femininity, and gender as a racialized feature (Pyke \& Johnson, 2003). Despite most participants' open resistance to gender oppression, many internalized this racialized-gendered construction of Asian femininity and distanced themselves from their heritage by claiming that they are outliers or the "black sheep" of their family. This perception of the "Asian" culture perpetuates misconceptions about women's roles and expectations in the Western culture, while negating the dominance of patriarchal structures maintained in most contemporary institutions or the rich diversity within their own ethnic communities (Vu \& Rook, 2012). 
Central to Berry's acculturation theory is the assumption that mainstream and heritage cultures share equal status and power (Bhatia \& Ram, 2009). However, our findings do not support this claim. When considered alongside the more 'liberal' norms and values of the mainstream "Canadian" culture, most participants frequently perceived their "Asian" culture to be 'rigid' and 'old-fashioned'. For instance, participants' parents, whose own experiences of racism, social exclusion, and/or financial instability shaped the way they interacted with their children, were portrayed as 'restrictive and authoritative'. Thus, we argue that participants were more compelled and motivated to identify with the mainstream culture due to its promising benefits and opportunities for material and social capital, individualism, and freedom from gendered constraints (Pyke \& Johnson, 2003).

Because some participants shared the desire for these same values, they self-identified as "white-washed", "Westernized", or "Canadian", which they perceived to be distinctly separate from their "Asian" identities. Ironically, as participants expressed preference to "Canadian" values due to its promise of freedom associated with individualism and independence, they also engaged in conforming their identities to stereotypes of White women and implicated themselves in sustaining Asian subordination and racial oppression. Identifying with hegemonic mainstream discourse by resisting "traditional Asian" behaviours and norms alludes to some notion of empowerment and agency in constructing their own identities (Pyke \& Johnson, 2003). However, this is done at the expense of denying their heritage, reinforcing internalized racism and perpetuating Asian stereotypes that harm the Asian communities.

In addition, repeated experiences of racial microaggression, cultural domination, Othering and lack of Asian representation and "celebration" in mainstream contexts made it more challenging for some participants to align with their heritage culture. We do not suggest that all 
of the young women rejected their "Asian" backgrounds; indeed, there were some who expressed cultural pride and advocated against racialized-sexist stereotypes (we expand on this later in the section). Our analysis, however, illustrates that the social construction of the "dominant" mainstream and "subordinate" heritage cultures influenced the motivation and degree to which participants' aligned or distanced their identities to such. Bidimensional acculturation theory largely undermines asymmetric power relations that shape individuals' sense of belonging or difference. It diverts attention away from the meso- and macro-level structures that impinge on individual-level "outcomes" (Viruell-Fuentes et al., 2012). When constructing their identities, participants do not only consider, 'do I belong?' but also question, 'do we belong?', referring to social division and racialization imposed on their heritage groups. Once again, we are not generalizing claims that young Asian women's identities are entirely contingent on their racialized-gendered positions and experiences of marginalization. Rather, we suggest that socially constructed subordinations add a layer of complexity to the process of identity construction and these processes contribute to stress.

The presumption that power relations are horizontal across all racial and ethnic groups supports the myth of "free will" (i.e. that individuals possess when choosing their "acculturation outcomes"), and construction of identities (Schwartz et al., 2010; Ngo; 2008). Indeed, most participants felt that they had to choose between their heritage and mainstream White Canadian cultures since simultaneous alignment to both led to identity conflict and tension. However, participants' narratives demonstrate that achieving higher levels of White "Canadian" acculturation is not always achievable, and can lead to a false sense of identity and belonging. Their "free will" is constrained by asymmetric power relations and structurally reinforced social, gender, and racial inequities. Even as they neutralize their "Asian" identities and claim to be 
integrated into the mainstream culture, they continue to be met with experiences of Othering that force them to acknowledge that they do not fully belong and question their perceived proximity to the "Canadian" identity (Bhatia \& Ram, 2009). These experiences also involve processes of learning about and interpreting their ascribed racialized status in the Canadian social fabric and influence the way they negotiate and construct their cross-cultural identities (Viruell-Fuentes, 2007). As participants shared, experiences of Othering in the form of incredulous questions (i.e., 'what part of Asia are you from?'), racial discrimination, microaggressions, and imposition of the role of cultural ambassadors consumed them emotionally and psychologically, and potentially increased their vulnerability to compromised mental health.

Regardless of how they self-identified, the participants' narratives reflected their complex "in-between" positioning primarily shaped by their heritage or mainstream culture. This positioning goes beyond existing acculturation research, which offers dichotomous perspectives that pose "Canadian" and "Asian" cultures in oppositional categories without accounting for the socio-political and historical power relations (Hogwarth, Wagner, Magnussen, \& Sammut, 2014). Furthermore, acculturation theories portray identities as stable and fixed, rather than situational and context dependent (Chirkov, 2009b). Our findings reveal that young Asian women's identity construction involved an ongoing process of interpretation, de-construction, and (re)construction across cultural boundaries, often dictated by social relations based on race, gender, class, or religion. Some grappled with the "in-between" space that imposed assumptions such as, "not Asian enough" or "not Canadian enough". Therefore, we problematize simplistic acculturation discourse that portray identities and "acculturation outcomes" as static and developed in isolation of their socio-political and historical contexts. 
As young Asian women employed situational selves to reconcile their worlds, they experienced mental health vulnerabilities that led to identity tensions, conflicts, exhaustion, and in some cases, a sense of empowerment. Nonetheless, young Asian women are not passive recipients of these mental health vulnerabilities. Within their constraints, participants exercised a certain degree of agency when constructing their identities to achieve certain outcomes or determine how to manage experiences of oppression (Sundar, 2008). While some young women responded to Othering experiences through heritage distancing and rejection, others employed strategies to actively resist socio-culturally constructed hierarchies. Tapping into social resources within their families and ethnic communities provided some young Asian women access to conditions that fostered positive heritage connections. More importantly, these conditions supported the participants' ability to create unique cross-cultural spaces and a sense of self that is not contingent on any socially constricted "identity".

\subsection{Study Limitations}

While the findings of this study contribute to research on young Asian women's identity construction and mental health vulnerabilities by interrogating discourses of culture and acculturation, there are some limitations to consider. First, "Asians" represent a heterogeneous ethnic community comprised of multiple sub-groups diverse in socio-cultural, political, economic, and historical backgrounds. The exploratory nature of this study limits the number of participants. Our 14 participants were not inclusive of all Asian sub-groups. There were also more self-identified East Asian individuals (namely, Chinese and Korean) than those of South or South-East Asian backgrounds. However, as an exploratory qualitative study, our goal was not to provide generalizations about young Asian women's identities and mental well-being, but to 
offer new understanding to challenge the dominant discourses in existing literature on acculturation and enculturation.

Second, although multiple recruitment strategies were employed to be inclusive of young Asian women from all educational backgrounds, all of our participants were attending or graduated from secondary and post-secondary school. Future recruitment strategies need to be diversified to include participants with different educational levels.

Third, the original CIV study focused on cultures, identity construction, and sexuality of young Asian women, and not explicitly on their mental health or related vulnerabilities. This focus limited the breadth and depth of narratives specific to mental health. However, participants' narratives on their growing-up experiences and identity construction suggested that their experiences of being Othered and the demands to occupy in-between spaces had significant impact on their mental and emotional health.

Lastly, this study took place in the White settler society of Canada, specifically in a large metropolitan city where $37.4 \%$ of immigrants choose to settle (Statistics Canada, 2018c). Thus, the applicability of our findings to other young Asian women living in rural settings or smaller Canadian cities may be limited. However, the strength of this study and the methodology used rests on its capability to draw out unique participant interactions and dialogue within this group context, while fostering an in-depth understanding of their growing-up experiences (Daley, 2013).

It is beyond the scope of this study to explore within and across sub-group differences among young Asian women's experiences of acculturation and identity construction. Nonetheless, findings may contribute to the deconstruction of culture and acculturation and shed 
a critical light when exploring their experiences navigating cross-cultural identity construction and mental health vulnerabilities.

\subsection{Implications for Nursing Practice}

Transformative and ethical nursing practice must be built on a solid understanding of social justice and power relations beyond exercising self-awareness of one's own beliefs, values and attitudes. To do this, nurses and health care professionals must engage in ongoing critical reflexivity to unpack their current knowledge of power relations and the intersecting impact of race, gender, and class in shaping identities and health outcomes (McGibbon \& Etowa, 2009). This reflexive practice must also occur within existing nursing education, which are replete with “culturally-competent" frameworks that seldom interrogate structural inequity (Duffy, 2001). Without this critical perspective, we risk essentializing culture and identity, providing care that is based on oppressive stereotypes, ascribing mental health outcomes to individuals" "culture" and “acculturation strategies", putting blame on individuals with mental health challenges, and reinforcing individual-level health interventions that negate structural determinants of health (Viruell-Fuentes, 2007). Our findings challenge these assumptions by making visible that young Asian women's mental health vulnerabilities are shaped by unequal power relations based on gender, racial, and class locations. As such, mental health policy and practice must consider broader systemic contexts that shape the individual and collective experiences of Asian and other racialized women in diaspora. More research is also needed to examine strategies of resilience and resistance used by racialized women to overcome systemic exclusion as they construct their identities. 


\subsection{Conclusion}

Young Asian women's identity construction and "acculturation" and "enculturation" outcomes are embedded in complex power relations within their heritage and mainstream contexts. Our findings offer conceptual alternatives by suggesting that dominant discourses of “Asian” and "Canadian” cultures shape ones' access, ability, and motivation to achieve certain "acculturation outcomes". Conventional discourses of "acculturation" and "culture" undermine the role of structural inequities and Othering processes in shaping young Asian women's mental well-being. The application of acculturation theories in research can benefit from the integration of structural analysis using critical race and critical feminist theories. 


\section{Chapter 6: Conclusion}

\subsection{Introduction}

In this final chapter, I present an overview of the key insights gained from this study. I also summarize limitations to consider when interpreting these insights. Lastly, I draw on these findings to offer implications for nursing practice, education, and research in the field of “culture", "acculturation" and young Asian women's mental well-being.

\subsection{Overview of Key Insights}

This thesis sought to expand the limited research on 1.5 and 2.0 generation young Asian women's growing up experiences and mental well-being in Canada. Specifically, we explored how participants made sense of their experiences within the context of racialized-sexism and unchallenged discourses of culture, acculturation, and enculturation. Their narratives highlighted how they negotiated, constructed, and performed their identities as they navigated cross-cultural spaces and asymmetric power relations.

The findings presented in Chapter 4 challenge prevailing assumptions about the influence of "Asian" cultural determinants in shaping the mental health vulnerabilities and well-being of young Asian women. Our analysis revealed how structural forces of racialized-sexism and Whiteness reinforced their socially constructed positions as "subordinated" racialized women. This imposed location (re)produced stereotypes of "Asian femininity" and experiences of microaggressions and internalized oppression, shaping their mental health vulnerabilities. In addition, their parents' own experiences of marginalization and social exclusion produced a sense of associated suffering, which either manifested as feelings of empowerment or reinforced internalized oppression. As highlighted in Chapter 2, this critical perspective remains nascent 
from dominant discourses that ascribe young Asian women's negative mental health outcomes to their "heritage cultural problems".

The findings presented in Chapter 5 build on the insights gained in Chapter 4. In the same vein, we critically analyzed assumptions perpetuated by dominant bidimensional acculturation theories on the identity construction and mental well-being of young Asian women. The participants' narratives revealed that identity construction involved ongoing negotiation and (re)construction within cross-cultural contexts, historically dictated by asymmetric power relations and Whiteness. Repeated experiences of Othering and dominant discourses of "Asian" and "Canadian" cultures influenced young Asian women's interactions with and interpretations of their identities. Their narratives also reveal that while they made attempts to "fit in" with their mainstream contexts, their locations as racialized-gendered Others made this outcome temporary or unachievable. Furthermore, the analysis revealed how some participants were rendered to "inbetween" spaces, wherein they were ascribed as "too Asian to be Canadian" and "too Canadian to be Asian”. Within these growing up contexts, young Asian women employed situational selves to adapt and reconcile their worlds, which led to mental health vulnerabilities and feelings of tension, conflict, or empowerment.

Overall, the application of CRT, CFT, and CNA throughout the study generated critical insights on the unique experiences of being a 1.5 and 2.0 generation young Asian woman in Canada. Not only did it reveal the influence of broader social inequalities on their identity construction and mental well-being, but also how it shaped their responses of resilience and resistance. Young Asian women's resilience and resistance was facilitated by access to certain conditions and resources. While some participants expressed heritage pride and rejected racialized-gender inequality, there were others whose strategies of resistance remained 
implicated in perpetuating negative Asian stereotypes. The use of CRT, CFT, and CNA highlights the powerful, yet unconscious, ways racialized communities become 'hooked' in upholding Whiteness and (re)producing asymmetric power relations.

\subsection{Research Significance}

This thesis offers an important theoretical and conceptual alternative to current discourses of "culture", "enculturation", and "acculturation". Grounded in critical qualitative methods, this research highlights the importance of understanding and explicating the racialized-gendered experiences of young Asian women by challenging perpetual, oppressive dichotomies.

Our research findings are relevant to nurses and healthcare professionals. We expand the predominantly marginalizing and incomplete scholarship of "cultural competency" in healthcare settings by explicating how asymmetric power relations shape "cultures", which in turn influence identity construction and mental well-being. By explicitly interrogating taken-for-granted concepts of Whiteness, asymmetric power relations, microaggressions, and internalized oppression, we challenge assumptions about "Asian" and "Canadian" cultures, which is often the basis of "culturally competent" frameworks. Our findings contribute to the scholarship on "cultural competency" and "acculturation/enculturation" by providing a critical approach that enable nurses to recognize the structural determinants of mental health among racialized women in diasporic communities.

\subsection{Limitations of the Study}

Specific study limitations were described in Chapters 4 and 5. The following section offers overarching limitations to be considered when interpreting the data. First, while every effort was made to engage a diverse group of young Asian women, the small sample size of our exploratory study limited our ability to engage young Asian women from all walks of life and ethnicity. Our 
intent is not to conflate the narratives of young Asian women to undermine their diverse social, historical, cultural, and economic backgrounds. Further, qualitative research does not aim to produce empirically generalizable claims. Instead, we sought to provide an in-depth and focused analysis on the nature of young Asian women's growing up experiences in one metropolitan Canadian city. Particularized knowledge gained from critical analysis of acculturation and enculturation theories in the context of young Asian women's experiences of gendered-racism, identity construction, and mental well-being can be used to inform future research.

Second, information on participant demographics and study setting has been provided for readers to compare and assess the social contexts in relation to other topics of inquiry. Future research that engages a larger sample of Asian women from different socioeconomic, educational, cultural, and religious backgrounds is needed to generate deeper insights on how these layers of identity would yield a different perspective on the topic. In light of the unique experiences and cultural diversity among this heterogeneous population, future research that focuses on one specific Asian sub-group (i.e. Vietnamese, Filipino, Indian) to capture their salient socio-historical, political, and cultural variations is also critical (Zhang \& Moradi, 2012).

Lastly, the study's narrative approach in exploring young Asian women's growing up experiences is both its strength and limitation. It has generated rich materials and critical insights on the young Asian women's experiences of racialized-sexism, acculturation, and enculturation in light of discourses of Whiteness and power relations. However, the qualitative approach limits the reach of a larger group of participants. Future studies can benefit from using mixed methods, wherein validated scales on acculturation and enculturation, based on individual as well as structural determinants, are used in conjunction with a narrative approach to generate a more 
comprehensive understanding on identity construction, mental health vulnerabilities, and resilience.

\subsection{Implications}

Specific implications were described in Chapters 4 and 5. In the following sub-sections, I draw on our key insights to offer implications for nursing practice, education, and future research. As a whole, findings point to the importance of aligning with and championing critical social justice in nursing education, practice, and research. Critical social justice attends to examinations about the production and maintenance of systemic power structures (Anderson et al., 2009). Using critical theories and CNA to explore young Asian women's identity construction and mental well-being challenged the otherwise taken-for-granted discourses about “culture", “acculturation”, and “enculturation”. This approach revealed how young Asian women's identity construction are negotiated and contested by issues of racialized-sexism, colonialism, Whiteness, and power relations across their mainstream and heritage contexts.

Similarly, critical social justice nursing is about bringing the invisible to the fore, which includes learning about and interrogating socio-political and historical contexts that are explicitly linked to institutionalized oppression, power relations, and health inequities (McGibbon, Mulaudzi, Didham, Barton, \& Sochan, 2014). Beyond this, critical social justice nursing requires nurses to engage this knowledge to open up space for racialized and marginalized voices in meaningful ways to promote health equity. It asks the question: How do we draw on the racialized-gendered experiences of young Asian women and the broader discourses that shape them in developing inclusive and holistic mental health programs? Although social justice is an ethical foundation for nurses in Canada and worldwide (Canadian Nurses Association, 2010), it remains a promising ideal that is difficult to operationalize in nursing curricula and practice 
(Mohammed, Cooke, Ezeonwu, \& Stevens, 2014; Anderson et al., 2009). The insights of this study to offer critical social justice-based implications for nursing education, practice, and research.

\subsubsection{Implications for Nursing Education}

I start this sub-section with implications for nursing education because I believe it is a vehicle to develop a solid foundation of critical social justice nursing. Nursing's historical reductionistic and empiricist foundations undermine the role of structural power relations in perpetuating health inequities (McGibbon et al., 2014). More than ever, nurses are called to provide "culturally competent" care for their clients. Specifically, prevailing discourses of "culture" and "cultural competency" in nursing curricula essentialize "culture" as fixed "characteristics" of certain individuals and communities (Duffy, 2001). These discourses also focus on cultural "differences", in ways that privilege White dominant middle- and upper-middle class cultures, which perpetuate stereotypes as generalizable ascriptions for "culture-bound" outcomes such as mental health problems (Wong \& Tsang, 2004) and draws attention away from deeper structures (such as institutional power relations) that shape "cultures" and mental health outcomes in the first place (Metzl \& Hansen, 2014).

If nurses are to practice critical social justice, they must recognize the limitations of the concept of "cultural competency" and its" function to perpetuate the myth of White neutrality and social inequality for racialized and marginalized communities. We must also interrogate the role of knowledge production and knowledge producers in privileging Whiteness and colonized ways of knowing (McGibbon et al., 2014). The integration of critical theories into nursing education can help students and nurses identify how structural violence, in the form of racialized-sexism, Whiteness, and unequal power relations are mutually implicated in 
perpetuating negative identity construction and mental health vulnerabilities for young Asian and other racialized women.

Nursing educators are charged with the task of reorienting nursing pedagogy by integrating critical social justice theories (such as critical race and feminist theories, anti-oppression, intersectionality, etc.) early in their curricula, not limited to community health courses (Hilario, Browne, \& McFaden, 2017). An early commitment to critical social justice nursing can provide students with a strong foundation of systems-level, upstream thinking that interrogates the "causes of the cause" of health outcomes (McGibbon et al., 2014). It fosters critical approach to examine prevailing "cultural competency" frameworks; shifting away from ascribing individual's health outcomes to "cultures" but instead, recognizing the role of power inequities in shaping such outcomes. There is an evolving body of knowledge on 'structural competency' that may be helpful in supporting nurses to recognize the downstream implications of upstream contexts and social inequities (Metzl \& Hansen, 2014). This approach is not intended to replace foundational nursing courses; rather, it is meant to compliment the curricula to promote an inclusive and more holistic approach to nursing praxis.

Providing the protected time and safe space for students to explore not only pathological contexts, but also sociocultural, economic, historical, and political issues that shape the health of individuals and communities is imperative (Mohammed et al., 2014). One way to foster students' critical consciousness is to normalize classroom dialogues about how power, racialized-sexism, and oppression function to privilege or disadvantage certain social groups (Sue, 2010). These dialogues must not emphasize and essentialize differences but rather, focus on the impact of social inequities on the collective nature of health outcomes. In order to do this, educators must 
also practice critical reflexivity to confront their own assumptions and acknowledge the inherent privilege associated with their positionings.

\subsubsection{Implications for Nursing Practice}

In alignment with critical social justice, I believe that systemic issues require systemic shifts. Sustainable changes are made possible only when asymmetric power relations are acknowledged and disrupted at micro-, meso-, and macro- levels. While I recognize that there are many barriers to addressing this challenge, there are many entry points for nurses and healthcare providers to integrate critical social justice into all realms of their praxis. The importance of practicing reflexivity has been highlighted in Chapters 4 and 5. Reflexive practice involves a critical exploration of our social locations in the context of our own identities, lifeworlds, and social determinants of health. This enables us to examine our potential roles in the creation and maintenance of power imbalances within the nurse-client/nurse-community relationship (McGibbon \& Etowa, 2009). It also calls us to acknowledge our own feelings of powerlessness without projecting it onto our clients or colleagues. Engaging in reflexive practice can support nurses' commitment to health equity by taking action to actively resist, acknowledge, or speak up against any form of oppression (McGibbon et al., 2014). Central to this practice is recognizing whose perspectives are being privileged and marginalized, and how we can use our position to restructure these relations in all practice contexts.

Critical social justice should not only be conceptualized as a vague, abstract theory (Anderson et al., 2009). Existing research promotes causative and prescriptive associations between acculturation and enculturation and the mental health outcomes of Asian individuals and communities. These unchallenged discourses reproduce socially constructed stereotypes about Asians in diaspora, reinforce the onus of "culture" on individual-level mental health outcomes, 
and ignore the broader contexts within which they are shaped. Without critical social justice, nurses can potentially reproduce asymmetric power relations, social exclusion, and discrimination in their everyday interactions with clients and colleagues (Anderson et al., 2009). Engaging in critical social justice practice asks: Where do I get my knowledge? Who benefits from this perspective? Whose perspectives are missing? How do these assumptions inform my nursing practice? For instance, researchers ascribe the negative mental health outcomes of Asian individuals and communities to their "Asian" values, which is also benchmarked to "Canadian" values. In the absence of critical social justice, such discourses remain unchallenged; undermining the role of internalized oppression, colonialism, microaggressions, and racializedsexism in shaping such mental health outcomes. More importantly, sustainable transformative change is made possible when we engage others. Ongoing dialogue, de- and re-construction of knowledge, and evaluation must also occur on a group level to promote collective action and accountability for equitable health practices and policies (McGibbon \& Etowa, 2009).

Ultimately, we need to provide structures and opportunities for meaningful involvement, self-care, self-awareness, and dialogue to optimize young Asian women's mental well-being and identity construction. Increasing awareness of and coordination across community organizations or programs that promote young racialized women's health and sense of self can facilitate mutual empowerment, a sense of belonging, and strengthen relationships.

\subsubsection{Implications for Future Research}

According to Reissman (2008), "any methodological standpoint is, by definition, partial, incomplete, and historically contingent," (p. 70). The use of narrative approach in this study provided us with deeper insights about how young Asian women navigated their "cultures", 
identities, and mental well-being. Our particular findings represent one set of possibilities among many others; a starting point of ongoing exploration and not the end.

Some suggestions for future research have been highlighted in Chapters 4 and 5 and the limitation section. Our exploratory study is limited in its ability to address the experiences of Asian sub-groups and young Asian men. Asians are a diverse population with unique sociocultural, historical, political, and economic contexts. While there is also great within-group diversity, research that is focused on one Asian ethnic community (i.e. Filipinos, Sri Lankan, Korean, etc.) may be helpful in to explicating their unique identity construction and mental health experiences in Canada. Furthermore, there is also limited studies that focused on the acculturation/enculturation experiences of Asian men. Our findings indicate that the identity construction and mental health of Asian women are interwoven with the experiences of their interaction with men in their communities. Thus, there is an urgent need to critically explore how Asian men negotiate their identities and mental well-being within the context broader social constructs and power relations.

Our insights also reveal that existing approaches to acculturation/enculturation research remain incomplete and inadequate in exploring the complexity of this experience for Asian individuals. It is beyond the scope of this study to provide an exhaustive list of recommendations to improve acculturation research; other researchers have taken this endeavour (see Chirkov, 2009a, Chirkov 2009b, Weinreich, 2009). However, our analysis revealed that future acculturation/enculturation research should not be isolated from broader social constructs and the socio-political and historical contexts in which it occurs. Our identity construction and mental health experiences are not abstracted from our heritage and mainstream communities. Prevailing acculturation discourses that focus individual-level "acculturation outcomes" undermine this 
reality and perpetuate undue emphasis on individual-level interventions. Future acculturation research must be deliberately grounded on critical social justice theories to centralize discourses of racialized-sexism, agency, power relations, and Whiteness.

Perhaps the most important research implication of this study is the importance of providing young Asian women a safe and non-judgmental space for meaningful engagement and dialogue. The participants shared how the focus group challenged their internalized stereotypes, fostered critical thinking, and promoted a sense of belonging and mutual respect among their coethnic peers. Future studies about young racialized women's identity construction and mental health should incorporate dialogical focus-group methodology into community participatory action research. This approach fosters critical dialogue to interrogate structural discourses of power and social inequality and mobilize collective strengths to develop meaningful initiatives for social equity (Khanlou \& Peter, 2005).

\subsection{Conclusion}

Through the lens of critical theories, this study contributes to a nuanced exploration of how socially constructed discourses and power relations shape young Asian women's racializedgendered experiences and mental well-being. It also interrogates prevailing acculturation, enculturation theories, which predominantly obscure the role of dominant discourses in (re)producing systems of oppression. Although structural contexts reinforced their "subordinated" positionings as racialized young women, they drew on various strategies of resilience and resistance to negotiate their "cultural" identities. I strongly believe that nurses play a critical role in challenging oppressive ideologies that impact the health of racialized and marginalized communities. Critical social justice principles must be intentionally brought to the center of our work to promote health equity and meaningful involvement of clients and 
communities, not only to enhance construction of positive cultural identities, but also to disrupt oppressive social structures. 


\section{Appendix A: Ryerson University Research Ethics Board Approval Letter}

Ryerson

University

\section{Ethics Application - No REB Review Required \\ 1 message}

rebchair@ryerson.ca <rebchair@ryerson.ca>

Thu, Dec 14, 2017 at 4:25 PM

To: maria.abulencia@ryerson.ca, jph.wong@ryerson.ca

Re: REB 2017-402 Exploration of young Asian women's acculturation experiences and their mental health

Dear Maria Krisel Abulencia,

The Research Ethics Board has determined that your protocol does not require its review.

Thank you for your application for ethics review for the above noted study. In your protocol you have indicated that you will be conducting research involving only secondary use of de-identified data, and the results will not generate identifiable information. Based on this information, the project falls under Article 2.4 of the TCPS2 (the federal guidelines governing research ethics) and does not require REB review. Should your proposed methodology change, please reapply to the REB for ethics review and approval. Good luck with the project.

If you have any questions regarding your submission or the review process, please do not hesitate to get in touch with the Research Ethics Board (contact information below).

Record respecting or associated with a research ethics application submitted to Ryerson University.

NOTE: This email account (rebchair@ryerson.ca) is monitored by multiple individuals. If you wish to contact a specific member of the Research Ethics Board, please do so directly.

Yours sincerely,

Dr. Patrizia Albanese, PhD

Chair, Ryerson University Research Ethics Board

(416)979-5000 ext. 6526

palbanes@soc.ryerson.ca

rebchair@ryerson.ca

http://www.ryerson.ca/research

Dr. Asher Alkoby, LL.B., PhD

Vice-Chair, Ryerson Research Ethics Board

(416)979-5000 ext. 2491

aalkoby@ryerson.ca

http://www.ryerson.ca/research

Toni Fletcher, MA

Research Ethics Co-Ordinator

(416)979-5000 ext. 7112

toni.fletcher@ryerson.ca

Zakiya Atcha, MSW

Research Ethics Co-Ordinator

(416)979-5000 ext. 4841

zakiya.atcha@ryerson.ca 


\section{References}

Abe-Kim, J., Takeuchi, D. T., Hong, S., Zane, N., Sue, N., Spencer, M.S., Appel, H., Nicdao, E. \& Alegria, M. (2007). Use of mental health-related services among immigrant and USborn Asian Americans: Results from the national Latino and Asian American study. American Journal of Public Health, 97(1), 91-98.

Abouguendia, M. \& Noels, K. (2001). General and acculturation-related daily hassles and psychological adjustment in first and second-generation South Asian immigrants to Canada. International Journal of Psychology, 36(3), 163-173. doi:

$10.1080 / 00207590042000137$.

Agar, M. \& Hobbs, J. R. (1982). Interpreting discourse: Coherence and the analysis of ethnographic interviews. Discourse Processes, 5, 1-32.

Alamilla, S. G., Kim, B. S., Walker, T., \& Sisson, F. R. (2017). Acculturation, enculturation, perceived racism and psychological symptoms among Asian American college students. Journal of Multicultural Counseling and Development, 45(1), 37-65. doi: 10.1002/jmcd. 12062

Alapack, R. J. (2011). Under siege: A woman's beauty and health. The Humanistic Psychologist, 39, 366-374. doi: 10.1080/08873267.2011.620853

Anderson, J. M., Rodney, P., Reimer-Kirkham, S., Browne, A. J., Khan, K. B., \& Lynam, J. (2009). Inequities in health and healthcare viewed through the ethical lens of critical social justice: Contextual knowledge for the global priorities ahead. Advances in Nursing Science, $32(4), 282-294$.

Augsberger, A., Yeung, A., Dougher, M., \& Hahm, H.C. (2015). Factors influencing the underutilization of mental health services among Asian American women with a history of 
depression and suicide. BMC Health Services Research, 15. doi: 10.1186/s12913-015$1191-7$

Bakan, A. B., \& Stasiulis, D. K. (1995). Making the match: Domestic placement agencies and the racialization of women's household work. Signs: Journal of Women and Culture in Society, 20(2), 303-335.

Berger, M. \& Sarnyai, Z. (2015). "More than skin deep": Stress neurobiology and mental health consequences of racial discrimination. Stress, 18(1), 1-10. doi: $10.3109 / 10253890.2014 .989204$

Bernstein, K.S., Park, S., Shin, J., Cho, S., \& Park, Y. (2011). Acculturation, discrimination, and depressive symptoms among Korean immigrants in New York City. Community Mental Health Journal, 47(1), 24-34. doi: 10.1007/s10597-009-9261-0

Berry, J.W. (1974). Psychological aspects of cultural pluralism: Unity and identity reconsidered. Topics in Culture Learning, 2, 17-22.

Berry, J. W. (1980). Acculturation as varieties of adaptation. In A. M. Padilla (Ed.), Acculturation: Theory, models, and some new findings (pp. 9-25). Boulder, CO: Westview Press.

Berry, J. W. (1997). Immigration, acculturation and adaptation. Applied Psychology: An International Review, 46(1), 5-68.

Bhatia, S. \& Ram, A. (2009). Theorizing identity in transnational and diaspora cultures: A critical approach to acculturation. International Journal of Intercultural Relations, 33, 140149. doi:10.1016/j.ijintrel.2008.12.009 
Bivens, D. (2005). What is Internalized Racism? In M. Potapchuk, S. Leiderman, D. Bivens, \& B. Major, Flipping the script: White privilege and community building (pp. 43-52). Retrieved from http://www.racialequitytools.org/resourcefiles/potapchuk1.pdf.

Bodislav, D. A., Bran, F., \& Iovitu, M. (2015). A review of the globalization process. Review of International Comparative Management, 16(4), 471-478.

Borrell, C., Artazcoz, L., Gil-Gonzalez, D., Perez, K., Vives-Cases, C., \& Rohlfs, I. (2011). Determinants of perceived sexism and their role on the association of sexism with mental health. Women \& Health, 51, 583-603. doi: 10.1080/03630242.2011.608416

Brady, J.L., Kaya, A., Iwamoto, D., Park, A., Fox, L., \& Moorhead, M. (2017). Asian American women's body image experiences: A qualitative intersectionality study. Psychology of Women Quarterly, 41(4), 479-496. doi: 10.1177/0361684317725311

Bushnik, T. (2016). Women in Canada: A gender-based statistical report. The health of girls and women. Retrieved from: http://www.statcan.gc.ca/pub/89-503-x/2015001/article/14324eng.pdf.

Calliste, A. \& Dei, G. J. S. (2000). Anti-racist feminism: Critical race and gender studies. Halifax, NS: Fernwood

Canadian Mental Health Association (2008). Mental health promotion in Ontario: A call to action. Retrieved from: http://ontario.cmha.ca/documents/mental-health-promotion-inontario-a-call-to-action/

Canadian Nurses Association. (2010). Social Justice: A Means to An End, An End In Itself. Retrieved from: http://www.cna-aiic.ca/sitecore\%20modules/web/ /media/cna/pagecontent/pdf-en/social_justice_2010_e.pdf 
Chae, M. H., \& Foley, P. F. (2010). Relationship of ethnic identity, acculturation, and psychological well-being among Chinese, Japanese and Korean Americans. Journal of Counseling \& Development, 88(4), 466-476. doi: 10.1002/j.1556-6678.2010.tb00047.x

Chang, T. K., Wong, Y. J., Liu, T., \& Tran, K. (2017). Asian American female college students' subjective femininity conceptualizations: Using a mixed-methods approach. Journal of Multicultural Counseling and Development, 45, 260-275. doi: 10.1002/jmcd.12077

Chen, A. W., Kazanjian, A., \& Wong, H. (2009). Why do Chinese Canadians not consult mental health services: Health status, language, or culture? Transcultural Psychiatry, 46(4), 623641. doi: $10.1177 / 1363461509351374$

Chen, H., Yarnal, C., Chick, G., \& Jablonski, N. (2018). Egg white or sun-kissed: A crosscultural exploration of skin color and women's leisure behavior. Sex Roles, 78(3-4), 255271. doi: $10.1007 / \mathrm{s} 11199-017-0785-4$

Cheng, P., Carter, R. T., \& Lee, D. Y. (2014). The relationship between racial identity status attitudes and acculturation among Chinese and Korean Americans: A criterion profile analysis. Journal of Multicultural Counseling and Development, 43, 97-108. doi: $10.1002 / j .2161-1912.2015 .00067 . x$

Chirkov, V. (2009a). Critical psychology of acculturation: What do we study and how do we study it, when we investigate acculturation? International Journal of Intercultural Relations, 33, 94-105. doi:10.1016/j.ijintrel.2008.12.004

Chirkov, V. (2009b). Summary of the criticism and of the potential ways to improved acculturation psychology. International Journal of Intercultural Relations, 33, 199-180. doi: $10.1016 /$ j.ijintrel.2009.03.005 
Choi, Y., Tan, K. P. H., Yasui, M., \& Hahm, H. C. (2016). Advancing understanding of acculturation for adolescents of Asian immigrants: Person-oriented analysis of acculturation strategy among Korean American youth. Journal of Youth and Adolescence, 45, 1380-1395. doi: 10.1007/s10964-016-0496-0

Choi, Y., Kim, T. Y., Pekelnicky, D. D., Kim, K., \& Kim, Y. S. (2017). Impact of youth cultural orientation on perception of family process and development among Korean Americans. Cultural Diversity and Ethnic Minority Psychology, 23(2), 244-257. doi: $10.1037 / \mathrm{cdp} 0000093$

Chouliaraki, L., \& Fairclough, N. (1999). Discourse in late modernity: Rethinking critical discourse analysis. Edinburgh: Edinburgh University Press.

Chui, T. \& Maheux, H. (2011). Visible Minority Women. Women in Canada: A Gender-based Statistical Report. Retrieved from: http://www.statcan.gc.ca/pub/89-503X/2010001/article/11527-eng.pdf

Chui, T. (2015). Immigrant Women. Women in Canada: A Gender-based Statistical Report. Retrieved from: http://www.statcan.gc.ca/pub/89-503-x/2010001/article/11528eng.htm\#a4a

Cokley, K., Hall-Clark, B., \& Hicks, D. (2011). Ethnic minority-majority status and mental health: The mediating role of perceived discrimination. Journal of Mental Health Counseling, 33(3), 243-263

Cole, E. R. (2009). Intersectionality and research in psychology. American Psychologist, 64(3), 170-180. doi: 10.1037/a0014564

Creswell, J. W. (2013). Qualitative inquiry and research design: Choosing among five approaches $\left(3^{\text {rd }}\right.$ ed.). Thousand Oaks, CA: Sage. 
Creswell, J. W. \& Poth, C. N. (2018). Qualitative inquiry and research design: Choosing among five approaches $\left(4^{\text {th }}\right.$ ed.) Thousand Oaks, CA: Sage.

Daley, A. M. (2013). Adolescent-friendly remedies for the challenges of focus group research. Western Journal of Nursing Research, 35(8), 1043-1059. doi: 10.1177/0193945913483881

David, E. J. R. (2011). Filipino-American postcolonial psychology: Oppression, colonial mentality, and decolonization. Bloomington, IN: AuthorHouse

David, E. J. R. \& Okazaki, S. (2006). The colonial mentality scale (CMS) for Filipino Americans: Scale construction and psychological implications. Journal of Counseling Psychology, 53(2), 241-252. doi: 10.1037/0022-0167.53.2.241

David, E. J. R. \& Derthick, A. O. (2013) What is internalized oppression, and so what? In E. J. R. David (Ed.), Internalized oppression: The psychology of marginalized groups. (pp. 130). New York, NY: Springer

Davydov, D. M., Stewart, R., Ritchie, K., \& Chaudieu, I. (2010). Resilience and mental health. Clinical Psychology Review, 30, 479-495. doi: 10.1016/j.cpr.2010.03.003

Derr, A. S. (2015). Mental health service use among immigrants in the United States: A systematic review. Psychiatric Services, 67(3), 265-274. doi: 10.1176/appi.ps.201500004

Dhariwal, A. \& Connolly, J. (2013). Experiences of homeland and diaspora south Asian youth: Westernizing processes of media and friends. Journal of Research on Adolescence, 23(1), 45-56. doi: 10.1111/j.1532-7795.2012.00803.x

Duffy, M. E. (2001). A critique of cultural education in nursing. Journal of Advanced Nursing, $36(4), 487-495$.

Essed, P. (1990). Everyday Racism: Reports from women in two cultures. Claremont, CA: Hunter House. 
Farver, J. A., Narang, S. K., \& Bhadha, B. R. (2002). East meets west: Ethnic identity, acculturation and conflict in Asian Indian families. Journal of Family Psychology, 16(3), 338-350. doi: 10.1037//0893-3200.16.3.338

Fernando, S. \& Rinaldi, J. (2017). Seeking equity: Disrupting a history of exclusionary immigration frameworks. Canadian Ethnic Studies, 49(3), 7-26. doi:

10.1353/ces.2017.0019

Flannery, W. P., Reise, S. P., \& Yu, J. (2001). An empirical comparison of acculturation models. Personality and Social Psychology Bulletin, 27, 1035-1045.

Ford, C. L. \& Airhihenbuwa, C. O. (2010). Critical race theory, race equity, and public health: Toward antiracism praxis. American Journal of Public Health, 100(S1), 30-35.

Freire, P. (1970). Pedagogy of the oppressed. New York, NY: Continuum

Frye, M. (1983). On being white: Thinking toward a feminist understanding of race and race supremacy. In T. Burg (Ed.), Politics of reality: Essays in feminist theory. New York, NY: Crossing Press.

Fuligni, Al., Witkow, M., \& Garcia, C. (2005). Ethnic identity and the academic adjustment of adolescents from Mexican, Chinese and European backgrounds. Developmental Psychology, 41, 799-811.

Fung, K. \& Wong, Y. R. (2007). Factors influencing attitudes towards seeking professional help among east and southeast Asian immigrant and refugee women. International Journal of Social Psychiatry, 53(3), 216-231. doi: 10.1177/0020764006074541

Garcia, G.M., David, E. J. R., \& Mapaye, J.C. (2018). Internalized racial oppression as a moderator of the relationship between experiences of racial discrimination and mental 
distress among Asians and Pacific Islanders. Asian American Journal of Psychology, Advance online publication. http://dx.doi.org/10.1037/aap0000124

Gartner, M., Kiang, L., \& Supple, A. (2014). Prospective links between ethnic socialization, ethnic and American identity, and well-being among Asian-American adolescents. Journal of Youth Adolescence, 43, 1715-1727. doi: 10.1007/s10964-013-0044-0

Gee, G.C., Ro, A., Shariff-Marco, S., \& Chae, D. (2009). Racial discrimination and health among Asian Americans: Evidence, assessment, and directions for future research. Epidemiologic Reviews, 31, 130-151. doi: 10.1093/epirev/mxp009

Goodenow, C. \& Espin, O. (1993). Identity choices in immigrant adolescent females. Journal of Adolescence, 28(109), 173-184.

Gordon, M. M. (1964). Assimilation in American life: The role of race, religion, and national origins. New York: Oxford University Press

Graham, L., Brown-Jeffy, S., Aronson, R., \& Stephens, C. (2011). Critical race theory as theoretical framework and analysis tool for population health research. Critical Public Health, 21(1), 81-93. doi: 10.1080/09581596.2010.493173

Grant, P. R. (2016). “Canadian, Eh?” An examination of the multidimensional structure and functions of the multidimensional structure and functions of the national identity of immigrants and of those raised in Canada. Canadian Ethnic Studies, 48(1), 45-75.

Gupta, A., Szymanski, D. M., \& Leong, F. T. L. (2011). The "model minority myth": Internalized racialism of positive stereotypes as correlates of psychological distress, and attitudes toward help-seeking. Asian American Journal of Psychology, 2(2), 101-114. doi: $10.1037 / \mathrm{a} 0024183$ 
Gupta, A., Leong, F., Valentine, J. \& Canada, D. (2013). A meta-analytic study: The relationship between acculturation and depression among Asian Americans. American Journal of Orthopsychiatry, 83(23), 372-385. doi; 10.1111/ajop.12018.

Hall, S. (1997). Representation: Cultural representations and signifying practices. Thousand Oaks, CA: Sage.

Hewitt, M. (1991). Woman, nature and power: Emancipatory themes in critical theory and feminist theology. Studies in Religion, 20(3), 267-279.

Hilario, C., Vo, D., Johnson, J., \& Saewyc, E. (2014). Acculturation, gender and mental health of Southeast Asian immigrant youth in Canada. Journal of Immigrant Minority Health, 16, 1121-1129. doi: 10.1007/s10903-014-9978-X

Hilario, C. T., Browne, A. J., \& McFaden, A. (2017). The influence of democratic racism in nursing inquiry. Nursing Inquiry, 25(1), 1-7. doi: 10.1111/nin.12213

Hilario, C. T., Oliffe, J. L., Wong, J. P., Browne, A. J., \& Johnson, J. L. (2018). “Just as Canadian as anyone else"? Experiences of second-class citizenship and the mental health of young immigrant and refugee men in Canada. American Journal of Men's Mental Health, 12(2), 210-220. doi: 10.1177/1557988317743384

Hill, T. D. \& Needham, B. L. (2013). Rethinking gender and mental health: A critical analysis of three propositions. Social Science and Medicine, 92, 83-91. doi:

10.1016/j.socscimed.2013.05.025

Ho, R. (2014). Do all Asians look alike?: Asian Canadians as model minorities. Studies on Asia $4(2), 78-107$. 
Hogwarth, C., Wagner, W., Magnussen, N., \& Sammut, G. (2014). "Its only other people who make me feel black": Acculturation identity, and agency in a multicultural community. Political Psychology, 35(1), 81-95. doi: 10.1 111/pops.1 2020

Holloway, I. \& Freshwater, D. (2007a). Vulnerable story telling: Narrative research in nursing. Journal of Research in Nursing, 12(6), 703-711. doi: 10.1177/17449871084669.

Holloway, I. \& Freshwater, D. (2007b). Narrative research in nursing. Oxford: Blackwell.

Hudon, T. (2015). Immigrant Women. Women in Canada: A Gender-based Statistical Report. Retrieved from http://www.statcan.gc.ca/pub/89-503-x/2015001/article/14217-eng.pdf.

Hwang, W. \& Ting, J. Y. (2008). Disaggregating the effects of acculturation and acculturative stress on the mental health of Asian Americans. Cultural Diversity and Ethnic Minority Psychology, 14(2), 147-154.

Islam, F., Khanlou, N., \& Tamim, H. (2014). South Asian populations in Canada: migration and mental health. BMC Psychiatry, 14(154), 1-13. doi: 10.1186/1471-244X-14-154

Kalibatseva, Z., Leong, F. T. L., Ham, E. H., Lannert, B. K., \& Chen, Y. (2017). Loss of face, intergenerational family conflict, and depression among Asian American and European American college students. Asian American Journal of Psychology, 8(2), 126-133. doi: 10.1037/aap0000067

Kenway, J. \& Bullen, E. (2011). Skin pedagogies and abject bodies. Sport, Education and Society, 16(3), 279-294. doi: 1080/13573322.2011.565961

Khan, M., Kobayashi, K., Lee, S. M., \& Vang, Z. (2015). (In)Visible minorities in Canadian health data and research. Population Change and Lifecourse Strategic Knowledge Cluster Discussion Paper Series, Vol. 3: Iss. 1, Article 5. 
Khan, M. \& Cottrell, M. (2017). Oh Canada, whose home and native land? Negotiating multicultural, Aboriginal and Canadian identity narratives. Education Matters, 5(1), 1-34.

Khanlou, N. \& Peter, E. (2005). Participatory action research: Considerations for ethical review. Social Science \& Medicine, 60, 2333-2340. doi: 10.1016/j.socscimed.2004.10.004

Kim, B. S. K., Brenner, B. R., Liang, C. T. \& Asay, P. A. (2003). A qualitative study of adaptation experiences of 1.5-generation Asian Americans. Cultural Diversity and Ethnic Minority Psychology, 9(2), 156-170. doi: 10.1037/1099-9809.9.2.156

Kim, B. S. K. (2007). Acculturation and enculturation. In F. Leong, A. Inman, A. Ebreo, L. Yang, L. Kinoshita, \& M. Fu (Eds.), Handbook of Asian American psychology, (2 ${ }^{\text {nd }}$ ed.) Racial and Ethnic Minority Psychology (REMP) Series (pp. 141-158). Thousand Oaks: CA: Sage

Kim, B. S. K., Ahn, A. J., \& Lam, N. A. (2009). Theories and research on acculturation and enculturation experiences among Asian American Families. In N. Trinh, Y.C. Cho, F. G. Lu, \& K. M. Sanders (Eds.), Handbook of mental health and acculturation in Asian American families (pp. 25-43). New York, NY: Humana Press

Kim, B. S. K. \& Omizo, M. M. (2010). Behavioral enculturation and acculturation, psychological functioning, and help-seeking attitudes among Asian American adolescents. Asian American Journal of Psychology, 1(3), 175-185. doi: 10.1037/a0021125

Kivel, P. (1996). Uprooting racism: How white people can work for racial justice. Gabriola Island, BC: New Society Press.

Kumas-Tan, Z., Beagan, B., Loppie, C., MacLeod, A., \& Frank, B. (2007). Measures of cultural competence: Examining hidden assumptions. Academic Medicine, 82(6), 548-557. 
Kwak, K. (2010). Self-development and relationships through acculturation. Culture \& Psychology, 16(3), 365-381. doi: 10.1177/135406X10371141.

Langdridge, D. (2007). Phenomenological psychology: Theory, research and method. Harlow, UK: Pearson.

Lee, D. L. \& Ahn, S. (2011). Racial discrimination and Asian mental health: A meta-analysis. The Counseling Psychologist, 39(3), 463-489. doi: 10.1177/0011000010381791

Lee, J. W. \& Hebert, Y. M. (2006). The meaning of being Canadian: A comparison between youth of immigrant and non-immigration origins. Canadian Journal of Education, 29(2), 497-520.

Lee, S., Juon, H., Martinez, G., Hsu, C., Robinson, E.S., Bawa, J., \& Ma, G. (2009). Model minority at risk: Expressed needs of mental health by Asian American young adults. Journal of Community Health, 34, 144-152. doi: 10.1007/s10900-008-9137-1

Lee, S. M. \& Edmonston, B. (2010). “Canadian” as national ethnic origin: Trends and implications. Canadian Ethnic Studies, 41(3), 77-108. doi: 10.1353/ces.2010.0040

Leininger, M. (1991). Becoming aware of types of health practitioners and cultural imposition. Journal of Transcultural Nursing, 2(2), 32-39. doi: 10.1177/104365969100200205

Leu, J., Walton, E., \& Takeuchi, D. (2011). Conceptualizing acculturation: Gender, family, and community reception influences on Asian immigrant mental health. American Journal of Community Psychology, 48(3-4), 168-180. doi: 10.1007/s10464-010-9360-7

Levesque, A. \& Li, H. (2014). The relationship between culture, health conceptions, and health practices. Journal of Cross-Cultural Psychology, 45(4), 628-645. doi: $10.1177 / 0022022113519855$ 
Li, J. (2009). Forging the future between two different worlds: Recent Chinese immigrant adolescents tell their cross-cultural experiences. Journal of Adolescent Research, 24(4), 477-504. doi: $10.1177 / 0743558409336750$

Lipsicas, C. B. \& Makinen, I. L. (2010). Immigration and suicidality in the young. Canadian Journal of Psychiatry, 55(5), 273-281.

Liu, C. M. \& Suyemoto, K. L. (2016). The effects of racism-related stress on Asian Americans: Anxiety and depression among different generational statuses. Asian American Journal of Psychology, 7(2), 137-146. doi: 10.1037/aap0000046

Macgregor, R. M. (2003). I am Canadian: National identity in beer commercials. The Journal of Popular Culture, 37(2), 276-286.

Madison, D. S. (2005). Critical ethnography: Methods, ethics and performance. Thousand Oaks, CA: Sage.

Matthews, J. (2002). An ambiguous juncture: Racism and the formation of Asian femininity. Australian Feminist Studies, 17(38), 207-219. doi: 10.1080/0816464022014797

McGibbon, E. A. \& Etowa, J. B. (2009). Anti-racist health care practice. Toronto, ON: Canadian Scholars' Press Inc.

McGibbon, E., Mulaudzi, F. M., Didham, P. Barton, S., \& Sochan, A. (2014). Toward decolonizing nursing: The colonization of nursing and strategies for increasing the counternarrative. Nursing Inquiry, 21(3), 179-191. doi: 10.1111/nin.12042

Mental Health Commission of Canada. (2016). Advancing the Mental Health Strategy for Canada: A Framework for Action (2017-2022), Ottawa, ON: Mental Health Commission of Canada. 
Metzl, J. M. \& Hansen, H. (2014). Structural competency: Theorizing a new medical engagement with stigma and inequality. Social Science \& Medicine, 103, 126-133. doi: 10.1016/j.socscimed.2013.06.032

Miller, M. J. (2007). A bilinear multidimensional measurement model of Asian acculturation and enculturation: Implications for counseling interventions, Journal of Counseling Psychology, 54(2), 118-131. doi: 10.1037/0022-0167.54.2.118

Miller, M. J., Yang, M., Kayi, H., Choi, N. \& Lim, R. (2011). Acculturation, enculturation, and Asian American college students' mental health and attitudes toward seeking professional psychological help. Journal of Counseling Psychology, 58(3), 346-357. doi: $10.1037 / \mathrm{a} 0023636$

Minister of Justice. (2014). Canada Multiculturalism Act. Retrieved from Justice Laws website: http://laws-lois.justice.gc.ca/PDF/C-18.7.pdf.

Mischler, E. G. (1990). Validation in inquiry-guided research: The role of exemplars in narrative studies. Harvard Educational Review, 60(4), 415-442.

Mohammed, S. A., Cooke, C. L., Ezeonwu, M., \& Stevens C. A. (2014). Sowing the seeds of change: Social justice praxis in undergraduate nursing education. Journal of Nursing Education, 53(9), 488-493. doi: 10.3928/01484834-20140805-03

Mukkamala, S. \& Suyemoto, K.L. (2018). Racialized sexism/Sexualized racism: A multimethod study of intersectional experiences of discrimination for Asian American women. Asian American Journal of Psychology, 9(1), 32-46. doi: 10.1037/aap0000104

Muller, A. E., \& Gubrium, E. (2016). Researcher linguistic vulnerability: A note on methodological implications. Qualitative Health Research, 26(1), 141-144. doi: $10.1177 / 1049732315613312$ 
Nadal, K. L. (2009). Colonialism: Societal and psychological impacts on Asian Americans and Pacific Islanders. In N. Tewari \& A. Alvarez (Eds.), Asian American psychology: Current perspectives (pp. 153-172). New York, NY: Psychology Press.

Nap, A., van Loon, A., Peen, J. van Schaik, D., Beekman, A. \& Dekker, J. (2014). The influence of acculturation on mental health and specialized mental healthcare for non-western migrants. International Journal of Social Psychiatry, 61(6), 530-538. Doi: $10.1177 / 0020764014561307$

Ngo, V. (2008). A critical examination of acculturation theories. Critical Social Work, 9(1). Retrieved from http://www1.uwindsor.ca/criticalsocialwork/a-critical-examination-ofacculturation-theories.

Paradies, Y., Ben, J., Denson, N., Elias, A., Priest, N., Pieterse, A., Gupta, A., Kelaher, M., \& Gee, G. (2015). Racism as a determinant of health: A systematic review and meta-analysis. PLoS One, 10(9), 1-48. doi: 10.1371/journal.pone.0138511

Patton, M. Q. (2002). Qualitative research \& evaluation methods (3rd ed.). Thousand Oaks, CA: SAGE Publications.

Pyke, K. \& Dang, T. (2003). "FOB" and "Whitewashed": Identity and internalized racism among second generation Asian Americans, Qualitative Sociology, 26(2), 147-172.

Pyke, K. \& Johnson, D. (2003). Asian American women and racialized femininities: "Doing” gender across cultural worlds. Gender \& Society, 17(1), 33-53. doi: $10.1177 / 0891243202238977$.

Rajiva, M. (2006). Brown girls, white worlds: Adolescence and the making of racialized selves. The Canadian Review of Sociology and Anthropology, 43(2), 165-183. 
Reddy-Best, K. L., Kane, L., Harmon, J., \& Gagliardi, N.R. (2018). Critical perspectives on fashion textbooks: Representations of race, gender, and body. International Journal of Fashion Design, Technology, and Education, 11(1), 63-75. doi:

$10.1080 / 17543266.2017 .1299226$

Redfield, R., Linton, R., \& Herskovitz, M. J. (1936). Memorandum for the study of acculturation. American Anthropologist, 38, 149-152.

Reissman, C. K. (1993). Narrative analysis. Newbury Park, CA: Sage.

Rhee, S., Chang, J., \& Rhee, J. (2003). Acculturation, communication patterns and self-esteem among Asian and Caucasian American adolescents. Adolescence, 38(152), 749-768.

Roy, J. (2008). Legalized racism. Toronto: Canadian Race Relations Foundation. Retrieved from http://www.crrf-fcrr.ca/images/stories/pdf/ePubFaShLegRac.pdf.

Rudmin, F. (2009). Constructs, measurements, and models of acculturation and acculturative stress. International Journal of Intercultural Relations, 33, 106-123. doi: 10.1016/j.ijintrel.2008.12.001

Ruzek, N. A., Nguyen, D. Q., \& Herzog, D. C. (2011). Acculturation, enculturation, psychological distress and help-seeking preferences among Asian American college students. Asian American Journal of Psychology, 2(3), 181-196. doi: 10.1037/a0024302

Ryabov, I. (2016). Colorism and educational outcomes of Asian Americans: Evidence from the National Longitudinal Study of Adolescent Health. Social Psychology of Education, 19(2), 202-324. doi: 10.1007/s11218-015-9327-5

Salas-Wright, C. P., Kagotho, N., \& Vaughan, M. G. (2014). Mood, anxiety, and personality disorders among first and second-generation immigrants to the United States. Psychiatry Research, 220(3), 1028-1036. doi: 10.1016/j.psychres.2014.08.045 
Sam, D. L. \& Berry, J. W. (2006). The Cambridge handbook of acculturation psychology. New York, NY: Cambridge University Press.

Samuel, L. (2010). Mating, dating and marriage: Intergenerational culture retention and construction of diasporic identities among South Asian immigrants in Canada. Journal of Intercultural Studies, 31(1), 95-110. 10.1080/07256860903477712.

Schwartz, S. J., Unger, J. B., Zamboanga, B. L., \& Szapocznik, J. (2010). Rethinking the concept of acculturation. American Psychologist, 65(4), 237-252. doi: 10.1037/a0019330.

Sharma, N. (2006). Home economics: Nationalism and the making of 'migrant workers' in Canada. Toronto, ON: University of Toronto Press.

Shea, M. \& Yeh, C. (2008). Asian American students' cultural values, stigma and relational selfconstrual: Correlates of attitudes toward professional help seeking. Journal of Mental Health Counseling, 30(2), 157-172.

Souto-Manning, M. (2014). Critical narrative analysis: The interplay of critical discourse and narrative analyses. International Journal of Qualitative Studies in Education, 27(2), 159180. doi: $10.1080 / 09518398.2012 .737046$

Stacey, G., Felton, A., Morgan, A., Stickley, T., Wills, M., Diamond, B., Houghton, P., Johnson, B., \& Dumenya, J. (2016). A critical narrative analysis of shared decision-making in acute inpatient health care. Journal of Interprofessional Care, 30(1), 35-41. doi: $10.3109 / 13561820.2015 .1064878$

Statistics Canada. (2016). 150 years of immigration in Canada. Retrieved from: http://www.statcan.gc.ca/pub/11-630-x/11-630-x2016006-eng.htm 
Statistics Canada. (2017). Immigration and ethnocultural diversity: Key results from the 2016 Census. Retrieved from: http://www.statcan.gc.ca/daily-quotidien/171025/dq171025beng.htm

Statistics Canada. (2018a). Generation status: Canadian-born children of immigrants. Retrieved from: https://www12.statcan.gc.ca/nhs-enm/2011/as-sa/99-010-x/99-010-x2011003_2eng.cfm

Statistics Canada. (2018b). Ethnic diversity and immigration. Retrieved from: https://www150.statcan.gc.ca/n1/pub/11-402-x/2011000/chap/imm/imm-eng.htm Statistics Canada. (2018c). Immigration and Ethnocultural Diversity in Canada. Retrieved from: https://www12.statcan.gc.ca/nhs-enm/2011/as-sa/99-010-x/99-010-x2011001-eng.cfm\#a4

Stevens, P. A. (2013). A little place in the (next) country: Negotiating nature and nation in 1970s Ontario. Journal of Canadian Studies, 47(3), 42-66.

Stroink, M. L. \& Lalonde, R. N. (2009). Bicultural identity conflict in second-generation Asian Canadians. The Journal of Social Psychology, 149(1), 44-65. doi: 10.3200/SOCP.149.1.4465

Suarez-Orozco, C. \& Qin, D.B. (2006). Gendered perspectives in psychology: Immigrant origin youth. International Migration Review, 40(1), 165-198. doi: 10.1111/j.17477379.2006.00007.x

Sue, D. W. (2010). Microaggressions in everyday life: Race, gender, and sexual orientation. Hoboken, NJ: Wiley.

Sue, D. W., Bucceri, J., Lin, A. I., Nadal, K. L., \& Torino, G. C. (2007). Racial microaggressions and the Asian experience. Cultural Diversity and Ethnic Minority Psychology, 13(1), 7281. doi: 10.1037/1099-9809.13.1.72 
Sue, D. W., Capodilupo, C. M., \& Holder, A. (2008). Racial microaggressions in the life experience of Black Americans. Professional Psychology: Research and Practice, 39, 329336.

Suinn, R. (2010). Reviewing acculturation and Asian Americans: How acculturation affects health, adjustment, school achievement, and counseling. American Journal of Psychology, 1(1), 5-7. doi: 10.1037/a001879

Sundar, P. (2008). To "brown it up" or to "bring down the brown": Identity and strategy in second-generation, south Asian-Canadian youth. Journal of Ethnic \& Cultural Diversity in Social Work, 17(3), 251-278. doi: 10.1080/15313200802258166

Syed, M. \& Juan, M. J. (2012). Discrimination and psychological distress: Examining the moderating role of social context in a nationally representative sample of Asian American adults. Asian American Journal of Psychology, 3(2), 104-120. doi: 10.1037/a0025275

Takeuchi, D., T., Zane, N., Hong, S., Chae, D. H., Gong, F., Gee, G. C., Walton, E., Sue, D., \& Alegria, M. (2007). Immigration-related factors and mental disorders among Asian Americans. American Journal of Public Health, 97(1), 84-90.

Tannock, S. (2011). Points of prejudice: Education-based discrimination in Canada's immigration system. Antipode, 43(4), 1330-1356. doi: 10.1111/j.1467-8330.2010.00864.x

Teranishi, R., Behringer, L., Grey, E. \& Parker, T. (2009). Critical race theory and research on Asian Americans and pacific islanders in higher education. New Directions for Institutional Research, 142, 57-68. doi: 10.1002/ir.296

Tyson, L. (2014). Critical theory today: A user-friendly guide. (2 $2^{\text {nd }}$ ed.). New York, NY: Routledge. 
Tummala-Narra, P., Alegria, M., \& Chen, C. (2012). Perceived discrimination, acculturative stress, and depression among South Asians: Mixed findings. Asian American Journal of Psychology, 3(1), 3-16.

Tummala-Narra, P., Deshpande, A., \& Kaur, J. (2016). South Asian adolescents' experiences of acculturative stress and coping. American Journal of Orthopsychiatry, 86(2), 194-211, doi: $10.1037 /$ ort0000147

U.S. Department of Health \& Human Services. (2017). What is Mental Health? Retrieved from: https://www.mentalhealth.gov/basics/what-is-mental-health

Vaghela, P. \& Ueno, K. (2017). Racial-ethnic identity pairings and mental health of secondgeneration Asian adolescents. Sociological Perspectives, 60(4), 834-852. doi: $10.1177 / 0731121416683159$

Viruell-Fuentes, E. A. (2007). Beyond acculturation: immigration, discrimination, and health research among Mexicans in the United States. Social Science \& Medicine, 65(7), 15241535. doi: $10.1016 /$ j.socscimed.2007.05.010

Viruell-Fuentes, E. A. (2011). "It's a lot of work": Racialization processes, ethnic identity formations, and their health implications. Du Bois Review: Social Science Research on Race, 8(1), 37-52.

Viruell-Fuentes, E. A., Miranda, P. Y., \& Abdulrahim, S. (2012). More than culture: Structural racism, intersectionality theory, and immigrant health. Social Science \& Medicine, 75, 2099-2106. doi: 10.1016/j.socscimed.2011.12.037

Vu, H. Q. \& Rook, K. S. (2012). Acculturation and intergenerational relationships in Vietnamese families: The role of gender. Asian American Journal of Psychology, 4(3), 227-234. doi: $10.1037 / \mathrm{a} 0029750$ 
Watson, S. (2017). Everyday nationalism and international hockey: Contesting Canadian national identity. Nations and Nationalism, 23(2), 289-308. doi: 10.1111/nana.12163

Weinreich, P. (2009). 'Enculturation', not 'acculturation': Conceptualising and assessing identity processes in migrant communities. International Journal of Intercultural Relations, 33, 124-139. doi: 10.1016/j.ijintrel.2008.12.006

Wells, K. (2011). Narrative inquiry. New York, NY: Oxford University Press.

Williams, D. R. \& Mohammed, S. A. (2013). Racism and health I: Pathways and scientific evidence. American Behavioral Scientist, 57(8), 1152-1173. doi:

$10.1177 / 0002764213487340$

Wong, J. P. (2014). CHAMP research training on data analysis. Toronto, ON: Committee for Accessible AIDS Treatment (CAAT) CBR Research Training Series (unpublished).

Wong, Y. R. \& Tsang, A. K. T. (2004). When Asian immigrant women speak: From mental health to strategies of being. American Journal of Oropsychiatry, 74(4), 456-466. doi: $10.1037 / 0002-9432.74 .4 .456$

World Health Organization (2012). Risks to mental health: An overview of vulnerabilities and risk factors. Retrieved from: https://www.who.int/mental_health/mhgap/risks_to_mental_health_EN_27_08_12.pdf

World Health Organization (2014). Mental Health: a state of wellbeing. Retrieved from http://www.who.int/features/factfiles/mental_health/en/

Wu, Z., Schimmele, C. M., \& Hou, F. (2012). Self-perceived integration of immigrants and their children. Canadian Journal of Sociology, 37(4), 381-408. 
Ying, Y. \& Han, M. (2007). The longitudinal effect of intergenerational gap in acculturation on conflict and mental health in south east Asian American adolescents. American Journal of Orthopsychiatry, 77(1), 61-66. doi: 1037/0002-9432.77.1.61

Yoon, E., Chang, C., Kim, S., Clawson, A., Cleary, S. E., Hansen, M. Bruner, J. P., Chan, T. K. \& Gomes, A. M. (2013). A meta-analysis of acculturation/enculturation and mental health. Journal of Counseling Psychology, 60(1), 15-30. doi:10.1037/a0030652

Yoon, E., Adams, K., Clawson, A., Chang, H., Surya, S., \& Jeremie-Brink, G. (2017). East Asian adolescents' ethnic identity development and cultural integration: A qualitative investigation. Journal of Counseling Psychology, 64(1), 65-79. doi: 10.1037/cou0000181

Young, C. B., Fang, D. Z., \& Zisook, S. (2010). Depression in Asian-American and Caucasian undergraduate students. Journal of Affective Disorders, 125(1-3), 379-382. doi: 10.1016/j.jad.2010.02.124

Zhang, S. \& Moradi, B. (2012). Asian American acculturation and enculturation: Construct clarification and measurement consolidation. The Counseling Psychologist, 41(5), 750790. doi:10.1177/0011000012456882 